\title{
Macrodactylini (Coleoptera, Scarabaeidae, Melolonthinae): primary types of type species and taxonomic changes to the generic classification
}

\author{
Juares FUHRMANN ${ }^{1, *}$ \& Fernando Z. VAZ-DE-MELLO ${ }^{2}$ \\ ${ }^{1}$ Museu de Zoologia da Universidade de São Paulo, 04218-970 São Paulo-SP, Brazil. \\ ${ }^{2}$ Departamento de Biologia e Zoologia, Instituto de Biociências, \\ Universidade Federal de Mato Grosso, 78060-900 Cuiabá-MT, Brazil. \\ *Corresponding author: jufuhrmann@gmail.com \\ ${ }^{2}$ Email: vazdemello@gmail.com \\ ${ }^{1}$ urn:1sid:zoobank.org:author:CD1B347B-7C90-448D-A0BA-8EA7083CAF59 \\ ${ }^{2}$ urn:1sid:zoobank.org:author:2FF2B7D6-1A6B-43C1-9966-A1A949FB2B05
}

\section{Table of contents}

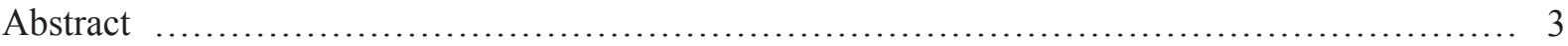

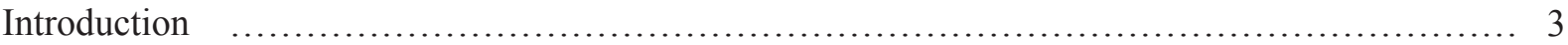

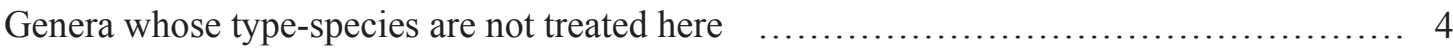

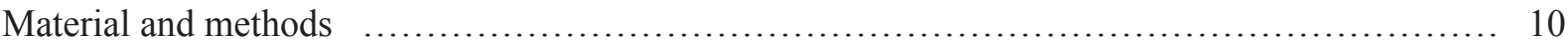

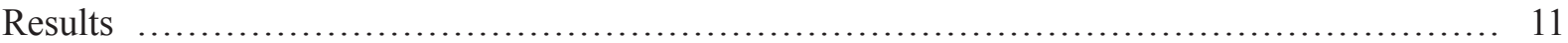

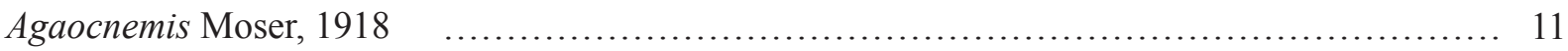

Agaocnemis pruina Moser, 1918 .................................................. 11

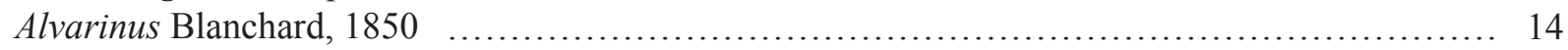

Alvarinus canescens (Burmeister, 1855) .......................................... 14

Ancistrosoma Curtis, 1835, nomen protectum ......................................... 14

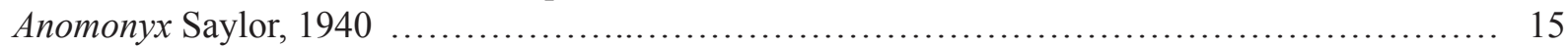

Anomonyx uruguayensis (Moser, 1921) ........................................ 16

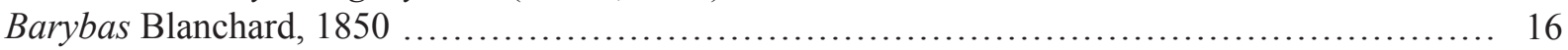

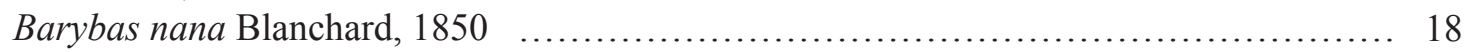

Barybas pellucens (Burmeister, 1855) _.......................................... 20

Barybas viridiaenea (Moser, 1821) …....................................... 20

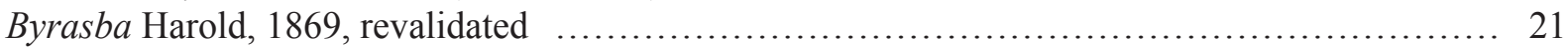

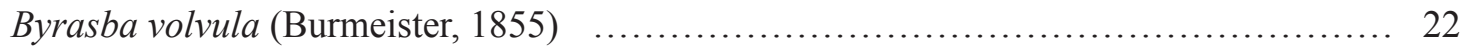

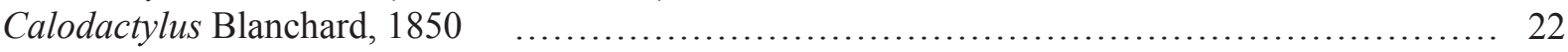

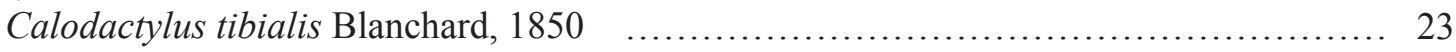

Ceraspis LePeletier de Saint-Fargeau \& Audinet-Serville, $1828 \quad$............................. 24

Ceraspis bivulnerata (Germar, 1824) …..................................... 25

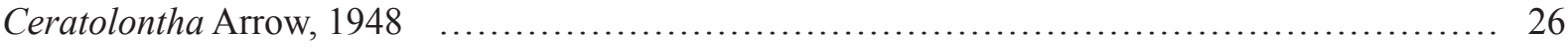

Ceratolontha venezuelae Arrow, 1948 ......................................... 28 


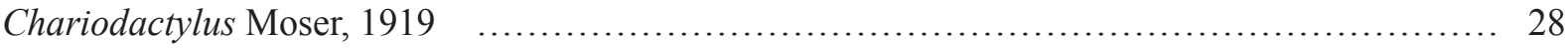

Chariodactylus chacoensis Moser, 1919 ...................................... 28

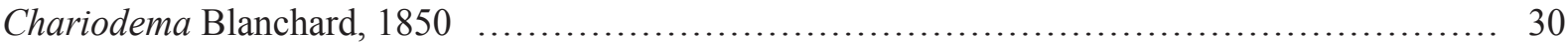

Chariodema virescens (Blanchard, 1842) …................................. 30

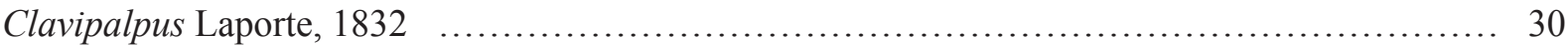

Clavipalpus dejeani Laporte, 1832 ............................................. 31

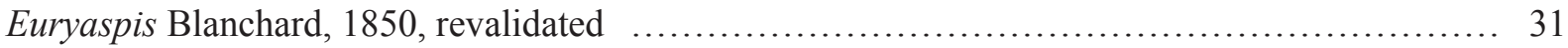

Euryaspis gaudichaudii Blanchard, 1850 ..................................... 32

Euryaspis obesa (Burmeister, 1855) comb. nov. ............................... 32

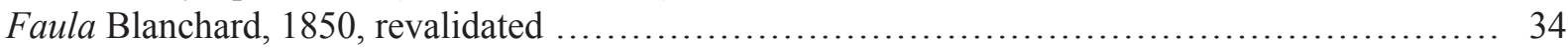

Faula cornuta Blanchard, 1850 ................................................ 34

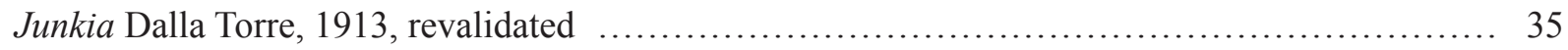

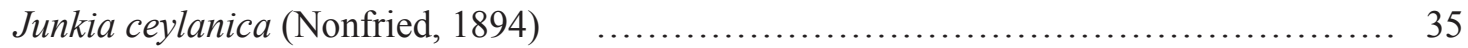

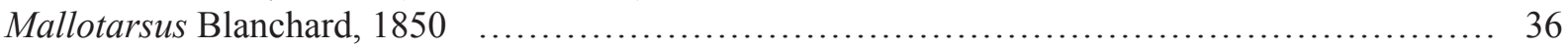

Mallotarsus spadiceus Blanchard, $1850 \quad$........................................ 36

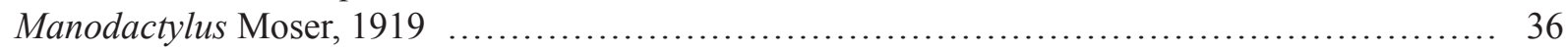

Manodactylus gaujoni Moser, 1919 ............................................... 37

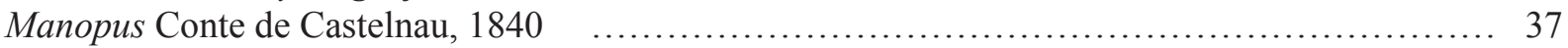

Manopus biguttatus Conte de Castelnau, 1840 .................................... 39

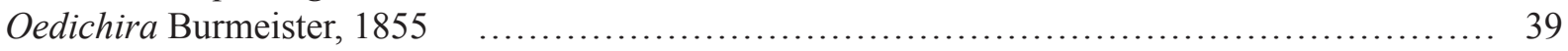

Oedichira pachydactyla Burmeister, $1855 \quad$..................................... 39

Paulosawaya Martínez \& d'Andretta, 1956, revalidated $\quad$.................................... 40

Paulosawaya ursina (Blanchard, 1850) comb. nov. $\quad$................................ 41

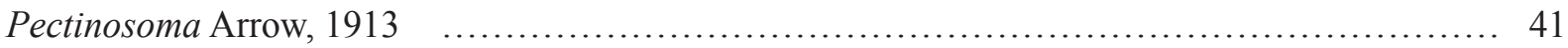

Pectisonoma elongate Arrow, $1913 \quad$.............................................. 42

Philochloenia Dejean, 1833 ............................................................. 42

Philochloenia armata Fuhrmann \& Vaz-de-Mello, 2017 nom. nov. ..................... 44

Philochloenia rufipennis (Fabricius, 1801) comb. nov …............................ 45

Philochloenia castaneipennis (Guérin-Méneville, 1831) comb. nov. ................. 45

Plectris LePeletier de Saint-Fargeau \& Audinet-Serville, 1828 ............................... 46

Plectris fallax (Blanchard, 1850) ................................................. 46

Plectris singularis (Blanchard, 1850) comb. nov. .................................. 47

Plectris tomentosa LePeletier de Saint-Fargeau \& Audinet-Serville, 1828 ............. 47

Pseudoserica Guérin-Méneville, 1838, revalidated .......................................... 48

Pseudoserica grandicornis (Blanchard, 1850) comb. nov. ......................... 48

Pseudoserica marmorea (Guérin-Méneville, 1838) .................................. 49

Pseudoserica viridis (Blanchard, 1850) comb. nov. .................................. 49

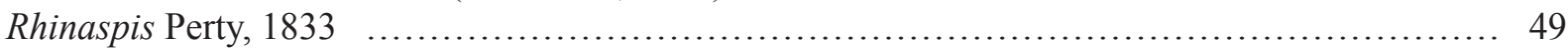

Rhinaspis aenofusca (Moser, 1919) ............................................. 50

Rhinaspis fuhrmanni Smith, 2016 ............................................ 51

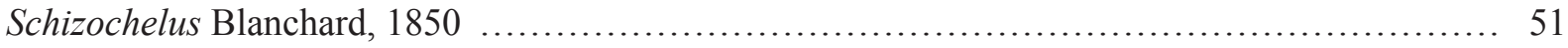

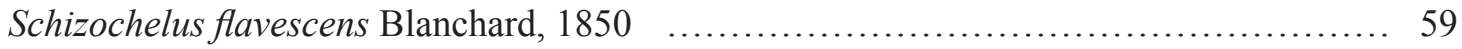

Schizochelus mirabilis (Moser, 1921) comb. nov. .................................. 60

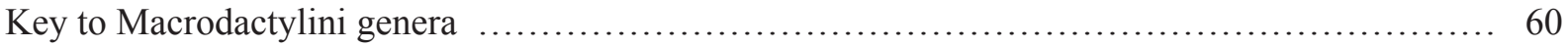

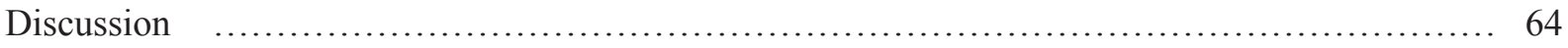

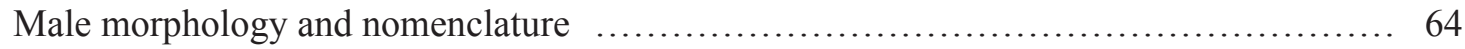

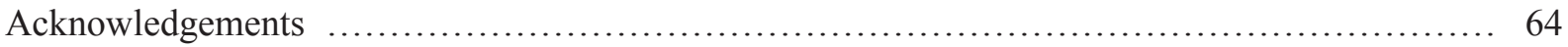

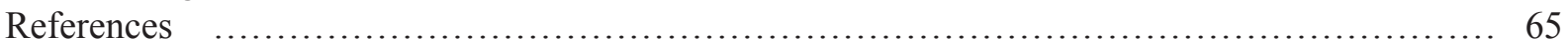


FUHRMANN J. \& VAZ-DE-MELLO F.Z., Type series of type species of Macrodactylini

Abstract. Type series for 35 type species of Macrodactylini (Coleoptera: Scarabaeidae: Melolonthinae) are studied and taxonomic changes are proposed. The following 35 lectotypes are designated: Agaocnemis pruina Moser, 1918; Amphicrania ursina Burmeister, 1855; Anomalochilus singularis Blanchard, 1850; Anomalonyx uruguayensis Moser, 1921; Aulanota sulcipennis Moser, 1924; Barybas nanus Blanchard, 1850; Barybas volvulus Burmeister, 1855; Calodactylus tibialis Blanchard, 1850; Ceraspis pruinosa LePeletier de Saint-Fargeau \& Audinet-Serville, 1828; Ceratolontha venezuelae Arrow, 1948; Chariodactylus chacoensis Moser, 1919; Clavipalpus dejeani Laporte, 1832; Corminus canescens Burmeister, 1855; Ctenotis obesa Burmeister, 1855; Ctilocephala pellucens Burmeister, 1855; Demodema fallax Blanchard, 1850; Euryaspis gaudichaudii Blanchard, 1851; Faula cornuta Blanchard, 1850; Gama grandicornis Blanchard, 1850; Gastrohoplus mirabilis Moser, 1921; Mallotarsus spadiceus Blanchard, 1850; Manodactylus gaujoni Moser, 1919; Manopus biguttatus Conte de Castelnau, 1840; Melolontha rufipennis Fabricius, 1801; Oedichira pachydactyla Burmeister, 1855; Pachycerus castaneipennis Guérin-Méneville, 1831; Pachylotoma viridis Blanchard, 1850; Pectinosoma elongata Arrow, 1913; Philochlaenia virescens Blanchard, 1842; Plectris tomentosa LePeletier de SaintFargeau \& Audinet-Serville, 1828; Pseudohercitis viridiaenea Moser, 1921; Rhinaspoides aeneofusca Moser, 1919; Schizochelus flavescens Blanchard, 1850; Serica marmorea Guérin-Méneville, 1831; and Ulomenes hypocrita Blanchard, 1850. The following six genera are revalidated: Byrasba Harold, 1869 (formerly a synonym of Rhinaspis Perty, 1833); Euryaspis Blanchard, 1851 (formerly a synonym of Plectris LePeletier de Saint-Fargeau \& Audinet-Serville, 1828); Junkia Dalla Torre, 1913 (formerly a synonym of Plectris); Faula Blanchard, 1850 (formerly a synonym of Ceraspis LePeletier de SaintFargeau \& Audinet-Serville, 1828); Paulosawaya Martínez \& d'Andretta, 1956 (formerly a synonym of Clavipalpus Laporte, 1832); and Pseudoserica Guérin-Méneville, 1838 (formerly a synonym of Plectris). The following 11 new generic synonymies are proposed: Anomalochilus Blanchard, 1850 a new synonym of Plectris; Amphicrania Burmeister, 1855 (formerly a synonym of Clavipalpus and a homonym of Amphicrania Dejean, 1833) and Pseudoleuretra Martínez \& d'Andretta, 1956 are synonymized with Paulosawaya; Aulanota Moser, 1924 and Hadrocerus Guérin-Méneville, 1838 are synonymized with Philochloenia; Ctenotis Burmeister, 1855 a new synonym of Euryaspis Blanchard, 1851; Gama Blanchard, 1850, Pachylotoma Blanchard, 1850 (formerly a synonym of Gama) and Harpodactyla Burmeister, 1855 (formerly a synonym of Gama) are synonymized with Pseudoserica; Gastrohoplus Moser, 1921 a new synonym of Schizochelus Blanchard, 1850; and Hercitis Burmeister, 1855 a new synonym of Barybas Blanchard, 1850. One new specific synonymy is proposed: Hercitis pygmaea Burmeister, 1855 a synonym of Barybas nana Blanchard, 1850. Philochloenia armata nom. nov. is proposed for Aulanota sulcipennis Moser, 1924 to avoid secondary homonymy. Ancistrosoma Curtis, 1835, nomen protectum, has priority over Sciuropus Dejean, 1833, nomen oblitum. Taxonomic remarks, diagnoses and a key are given to all Macrodactylini genera.

Keywords. Lectotype, Neotropical, Scarabaeoidea, systematic, type series.

Fuhrmann J. \& Vaz-de-Mello F.Z. 2017. Macrodactylini (Coleoptera, Scarabaeidae, Melolonthinae): primary types of type species and taxonomic changes to the generic classification. European Journal of Taxonomy 350: 1-71. https://doi.org/10.5852/ejt.2017.350

\section{Introduction}

Melolonthinae is among the most poorly known subfamilies within the Scarabaeoidea, and its tribal classification is in dire need of review (cf., Smith et al. 2006). Recent studies on the Macrodactylini have begun to address this problem. An important contribution to the Neotropical Melolonthinae was produced by Evans (2003), a checklist supplemented by Smith \& Evans (2005) and updated by Evans \& Smith $(2005,2007,2009)$. Katovich (2008) provided the first phylogenetic hypothesis for the tribe, and proposed a new view about the group. Since Katovich's (2008) Macrodactylini review, some changes to 
the generic classification were added by Smith (2008), who proposed taxonomic changes and described two genera (Ampliodactylus Smith, 2008 and Pusiodactylus Smith, 2008), by Katovich (2011) and Fuhrmann (2012), who described a genus each (Pseudopectinosoma Katovich, 2011 and Compsodactylus Fuhrmann, 2012), by Mondaca \& Ocampo (2012), who transferred Phyophis Redtenbacher, 1868 from Tanyproctini to Macrodactylini, and by Smith \& Mondaca (2015), who changed two genus placements (Modialis Fairmaire \& Germain, 1860 and Phytholaema Blanchard, 1851 to Macrodactylini) and described three genera (Extenuoptyophis Smith \& Mondaca, 2015, Insimuloissacaris Smith \& Mondaca, 2015 and Neuquenodactylus Smith \& Mondaca, 2015).

An evident problem within the tribe is that many genera have been characterized and taxonomic acts proposed without examination of their respective type series.

This paper proposes lectotype designations for the type species of 35 generic names $(21$ valid and 14 invalid names) of Macrodactylini, emends diagnoses, provides a key to genera, and corrects some nomenclatural problems that have persisted for more than a century. The importance of the boundary between taxon and name-bearing type as the objective standard of reference in modern taxonomy is stressed here and some important systematic problems are identified to encourage future studies.

Including the new changes proposed here, the Macrodactylini now comprises 46 Neotropical genera (Ceraspis LePeletier de Saint-Fargeau \& Audinet-Serville, 1828 with two subgenera) and 1028 species (Table 1).

\section{Genera whose type species are not treated here}

Macrodactylini now includes 85 available generic names, of which 46 are valid genera, one is a valid subgenus, and 38 are invalid names (Table 1). Five of them are replacement names (Anomonyx Saylor, 1940 for Anomalonyx Moser, 1921; Byrasba Harold, 1869 for Barybas Burmeister, 1855; Hadrocerus Guérin-Méneville, 1838 for Pachycerus Guérin-Méneville, 1831; Junkia Dalla Torre, 1913 for Trichoderma Nonfried, 1894; Pristerophora Harold, 1869 for Prionophora Solier, 1851), Macrodactylus Dejean, 1821 and Stenothorax Harris, 1827 have the same type species (Melolontha subspinosa Fabricius, 1775), and the other 79 generic names have type species (i.e., Macrodactylini includes 79 type species for generic names).

The present study designates 35 lectotypes for some of these 79 type species. The primary types of the other 44 macrodactiline type species are noted below.

The following five type species are being studied (articles in preparation): Alvarinus hilarii Blanchard, 1850 (type species of Alvarinus Blanchard, 1850, with four syntypes in MNHN) by Larissa Albuquerque (Universidade Federal de Pernambuco, Brazil) (pers. comm.); Canestera marshalli Saylor, 1938 (type species of Canestera Saylor, 1938, with a male holotype in BMNH) by J.F.; Dicrania nigra LePeletier de Saint-Fargeau \& Audinet-Serville, 1828 (type species of Dicrania, with a male syntype in MNHN) by J.F.; Hercitis pygmaea Burmeister, 1855 (type species of Hercitis Burmeister, 1855, and a new synonym of Barybas nana Blanchard, 1850, with a male syntype in ZNSM) by Holger Dombrow (pers. comm.); Monocrania luridipennis Laporte, 1832 (type species of Monocrania Laporte, 1832, a synonym of Dicrania, with a female syntype in MNHN) by J.F.

The depositories for the male holotypes are known for the following 11 type species: Astaenoplia miserabilis Martínez, 1957 (type species of Astaenoplia Martínez, 1957, holotype in the Antonio Martínez Collection, currently in MACN); Eubarybas asper Gutiérrez, 1952 (type species of Eubarybas Gutiérrez, 1952, and a synonym of Barybas, holotype in MNNC); Dicrania martinezi Frey, 1972 (type species of Compsodactylus, holotype in MACN); Extenuoptyophis metropolitensis Smith \& Mondaca, 


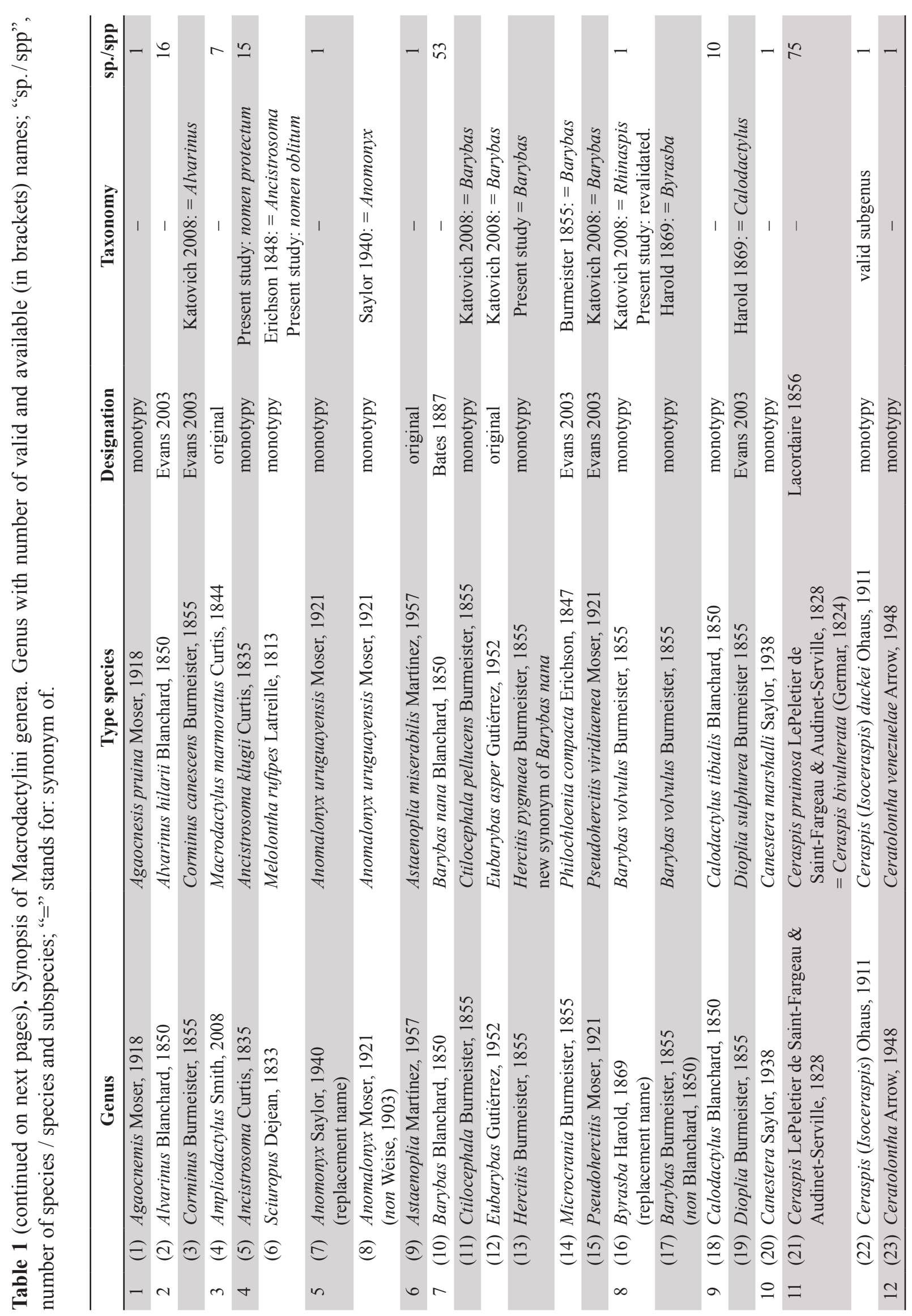



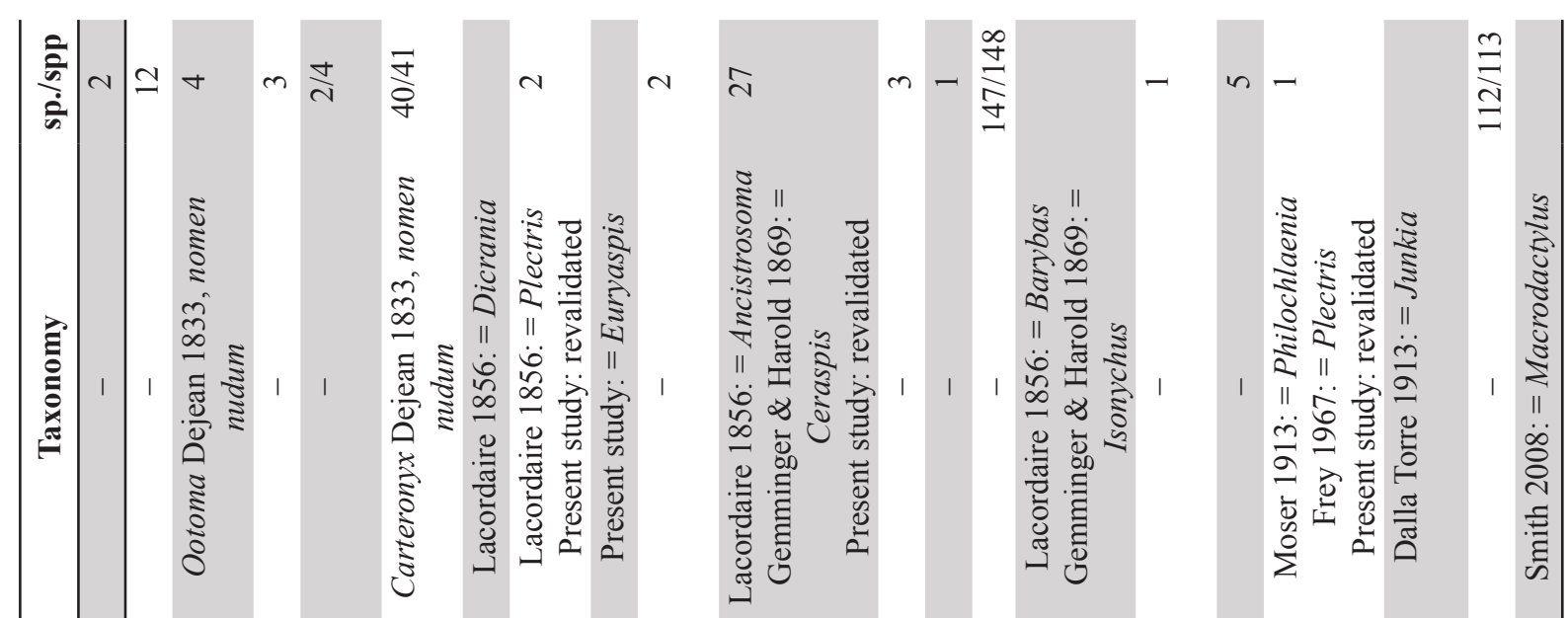

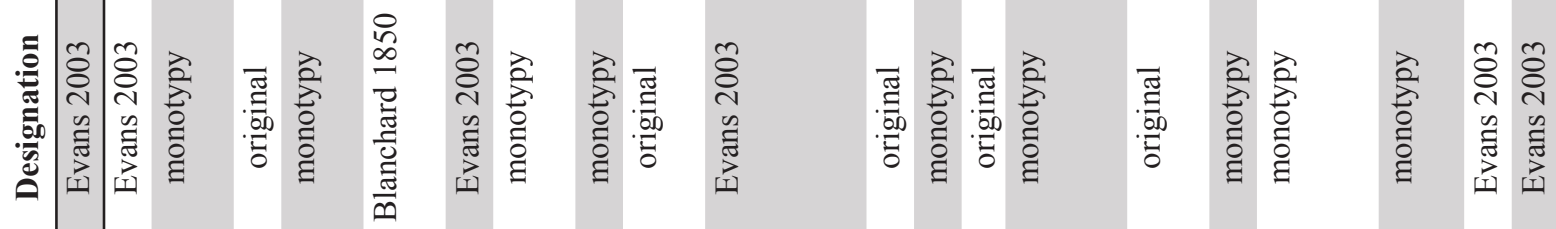
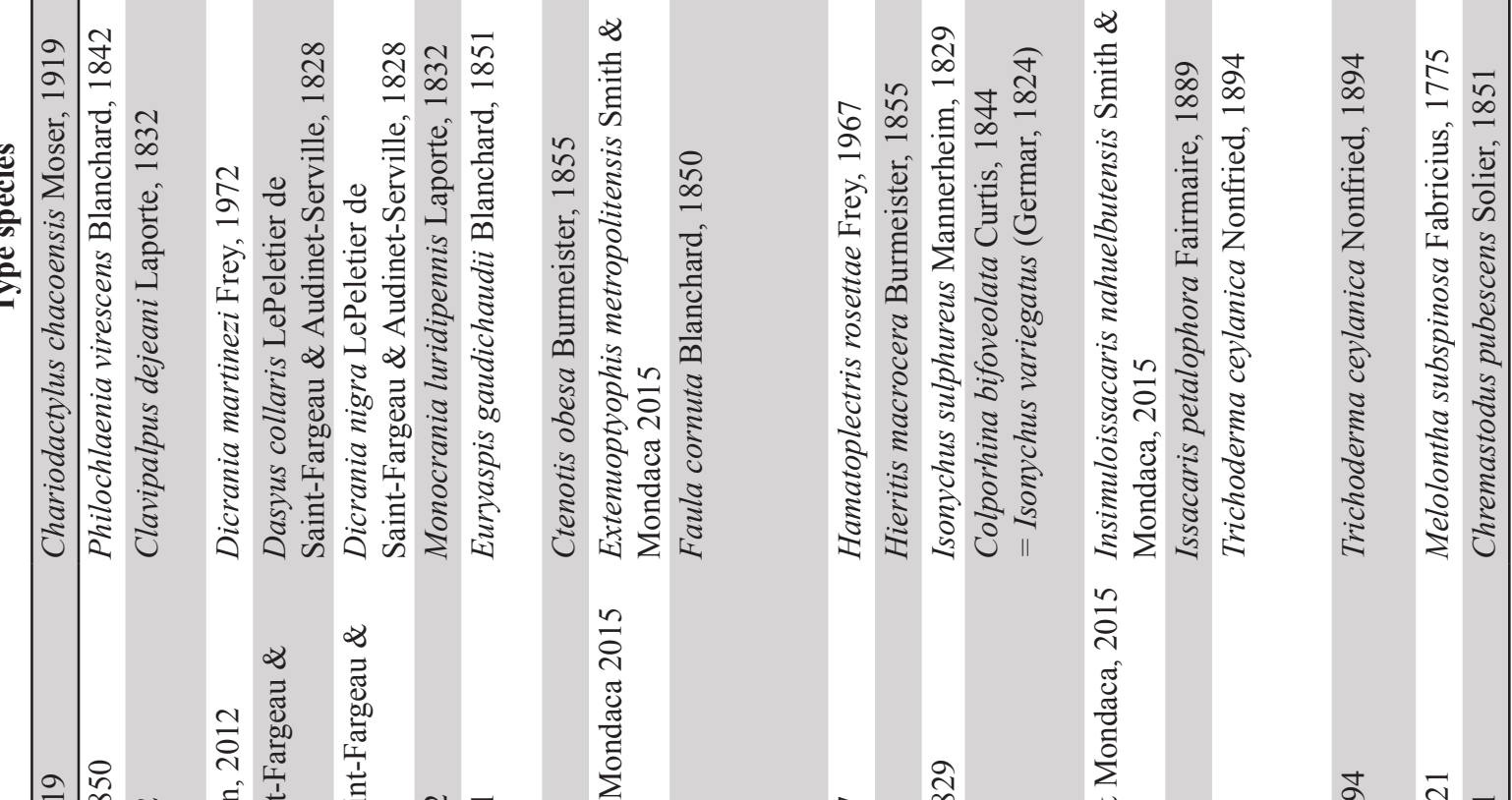

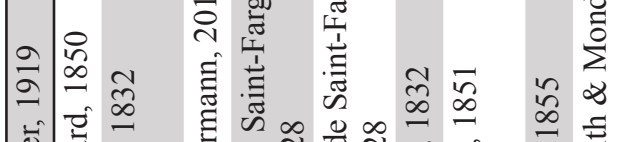

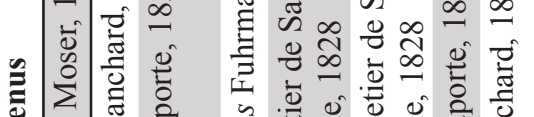

$\infty$ 音

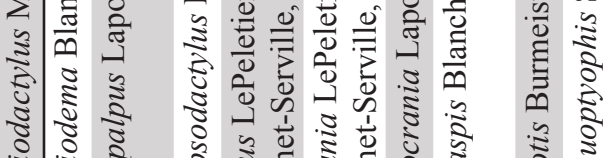

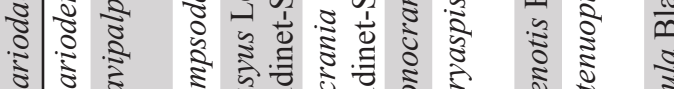

ปิ)

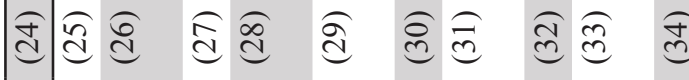

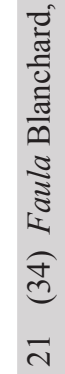

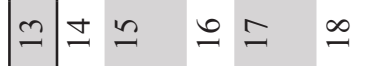

a

ก

ธิ $\underset{\infty}{\infty} \sum_{\infty}^{0}$

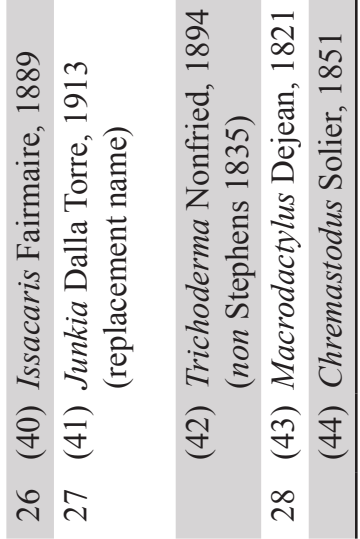




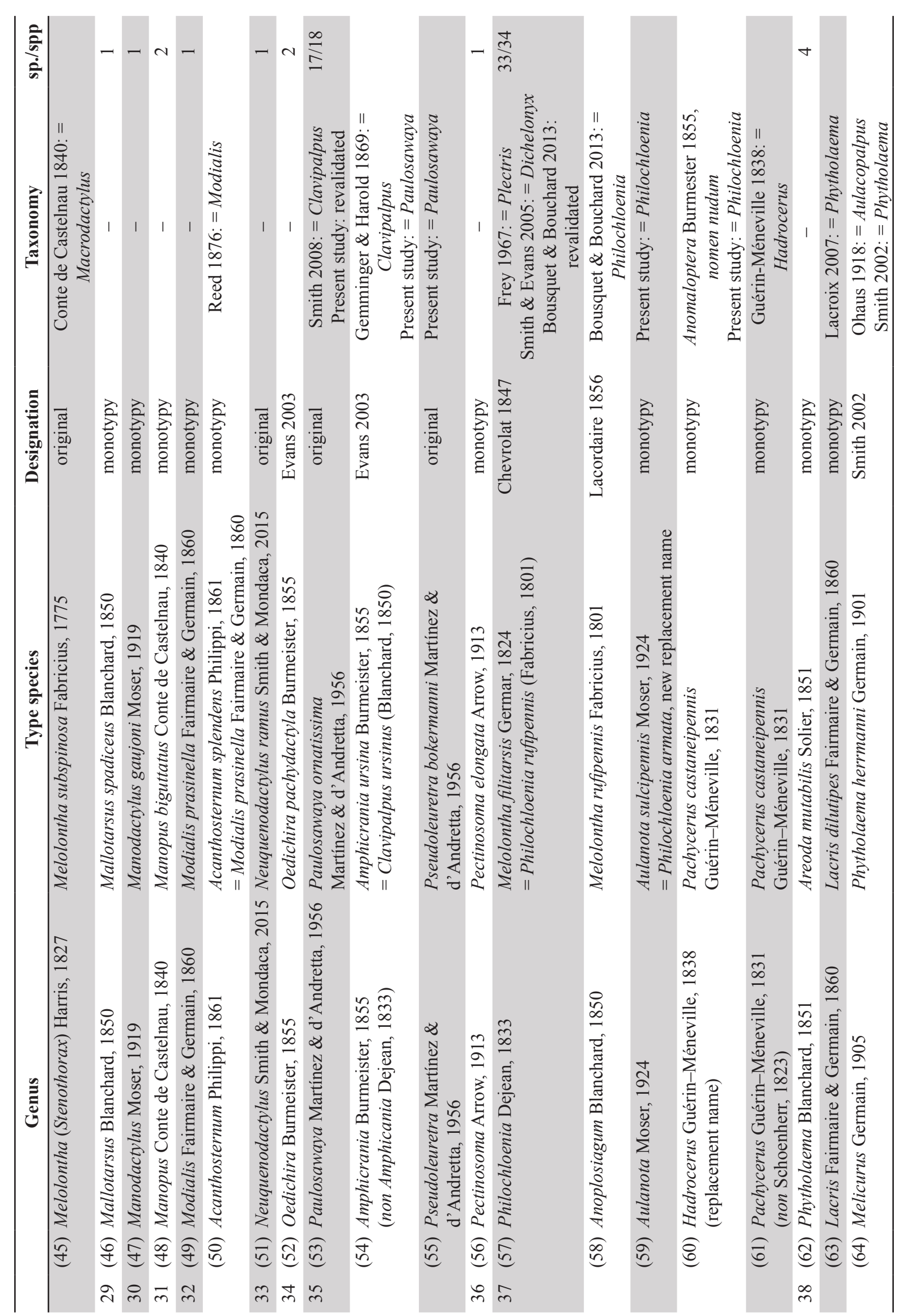




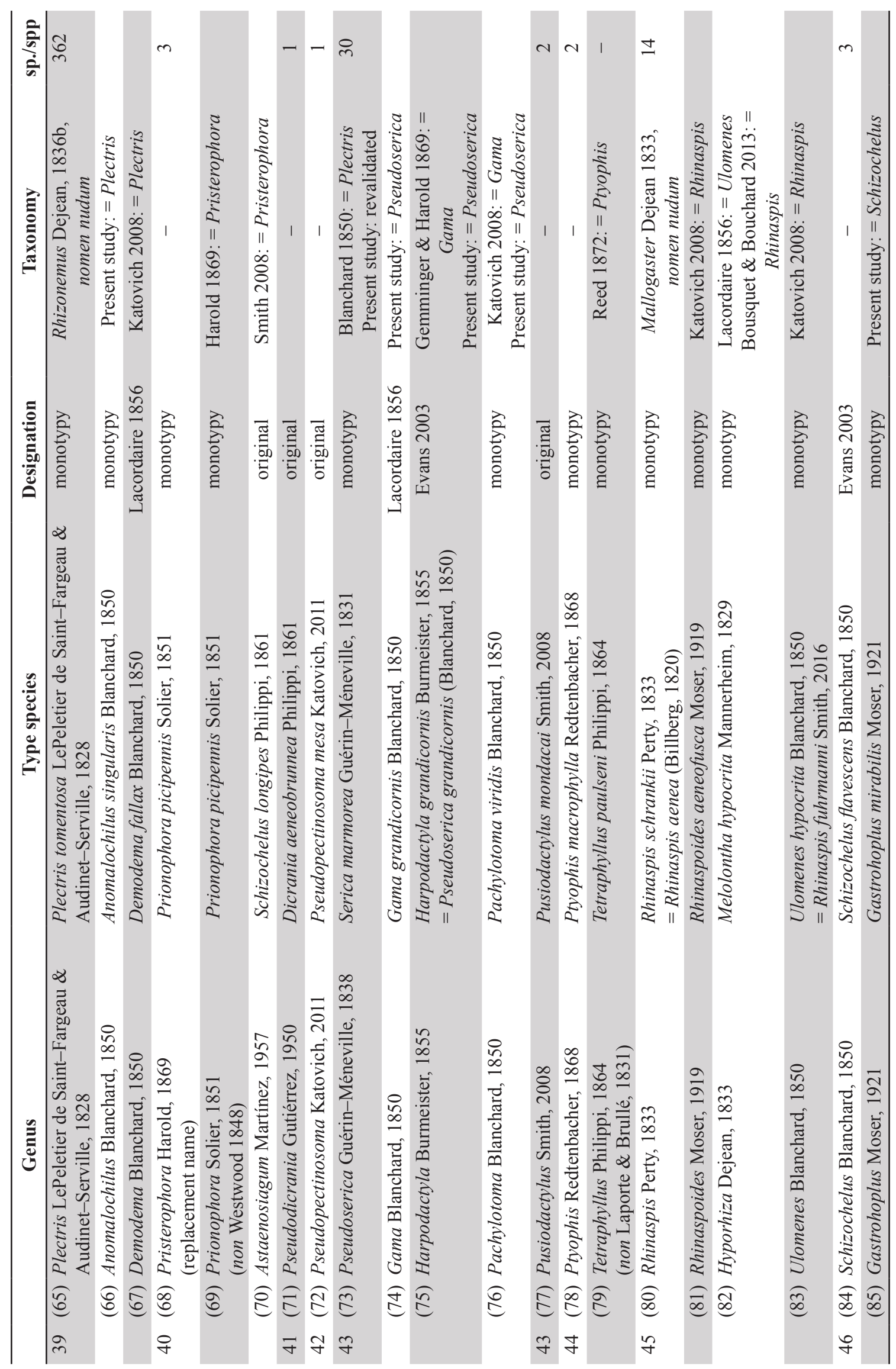


FUHRMANN J. \& VAZ-DE-MELLO F.Z., Type series of type species of Macrodactylini

2015 (type species of Extenuoptyophis, holotype in MNNC); Hamatoplectris rosettae Frey, 1967 (type species of Hamatoplectris Frey, 1967, holotype in NHMB); Insimuloissacaris nahuelbutensis Smith \& Mondaca, 2015 (type species of Insimuloissacaris, holotype in MNNC); Neuquenodactylus ramus Smith \& Mondaca, 2015 (type species of Neuquenodactylus, holotype in NHMW); Paulosawaya ornatissima Martínez \& d'Andretta, 1956 (type species of Paulosawaya Martínez \& d'Andretta, 1956, holotype in MZSP); Pseudoleuretra bokermanni Martínez \& d'Andretta, 1956 (type species of Pseudoleuretra Martínez \& d'Andretta, 1956, a new synonym of Paulosawaya, holotype in MZSP); Pseudopectinosoma mesa Katovich, 2011 (type species in Pseudopectinosoma, holotype in CMNC); Pusiodactylus mondacai Smith, 2008 (type species of Pusiodactylus, holotype in MNNC).

The following five studies designated primary types for 14 type species.

Lacroix (2007) designated a male lectotype (MNHN) for Lacris dilutipes Fairmaire \& Germain, 1860 (type species of Lacris Fairmaire \& Germain, 1860, a synonym of Phytholaema).

Smith (2008) designated lectotypes for the following four type species: Chremastodus pubescens Solier, 1851 (a synonym of Macrodactylus chilensis Solier, 1851, and the type species of Chremastodus Solier, 1851, a synonym of Macrodactylus, female lectotype in MNHN); Macrodactylus marmoratus Curtis, 1844 (type species of Ampliodactylus, male lectotype in BMNH); Prionophora picipennis Solier, 1851 (type species of Prionophora Solier now Pristerophora, male lectotype in MNHN); Schizochelus longipes Philippi, 1861 (type species of Astaenosiagum Martínez, 1957, a synonym of Pristerophora, male lectotype in MNNC). Thereafter, the author (Smith 2016) also designated a male lectotype for Melolontha hypocrita Mannerheim, 1829 (type species of Hyporhiza Dejean, 1833, a synonym of Rhinaspis Perty, 1833, lectotype in MNZH).

Mondaca \& Ocampo (2012) designated male primary types for the following two type species: Ptyophis macrophylla Redtenbacher, 1868 (type species of Ptyophis Redtenbacher, 1868, lectotype in NHMW); Tetraphyllus paulseni Philippi, 1864 (type species of Tetraphyllus Philippi, 1864, a synonym of Ptyophis, neotype in MNNC).

Smith \& Mondaca (2015) designated primary types for the following six type species: Acanthosternum splendens Philippi, 1861 (a synonym of Modialis prasinella Fairmaire \& Germain, 1860, and the type species of Acanthosternum Philippi, 1861, an objective synonym of Modialis, male neotype in MNHN); Areoda mutabilis Solier, 1851 (type species of Phytholaema, male neotype in MNNC); Dicrania aeneobrunnea Philippi, 1861 (type species of Pseudodicrania Gutiérrez, 1950, male lectotype in NMPC); Issacaris petalophora Fairmaire, 1889 (type species of Issacaris Fairmaire, 1889, male neotype in MNNC); Modialis prasinella Fairmaire \& Germain, 1860 (type species of Modialis, male lectotype in MNHN); Phytholaema herrmanni Germain, 1901 (type species of Melicurus Germain, 1905, a synonym of Phytholaema, male lectotype in MNNC).

The type series of the following 14 type species need to be studied: Ancistrosoma klugii Curtis, 1835 (type species of Ancistrosoma Curtis, 1835); Ceraspis (Isoceraspis) duckei Ohaus, 1911 (type species of Isoceraspis Ohaus, 1911, and a subgenus of Ceraspis); Colporhina bifoveolata Curtis, 1844 (a synonym of Isonychus variegatus (Germar, 1824), and type species of Colporhina Curtis, 1844, a synonym of Isonychus Mannerheim, 1829, see Barybas discussion); Dasyus collaris LePeletier de Saint-Fargeau \& Audinet-Serville, 1828 (type species of Dasyus LePeletier de Saint-Fargeau \& Audinet-Serville, 1828); Dioplia sulphurea Burmeister, 1855 (type species of Dioplia Burmeister, 1888, a synonym of Calodactylus Blanchard, 1850); Harpodactyla grandicornis Burmeister, 1855 (a synonym of Pseudoserica grandicornis (Blanchard), and type pecies of Harpodactyla Burmeister, 1855, a new synonym of Pseudoserica Guérin-Méneville, 1838); Hieritis macrocera Burmeister, 1855 (type species of Hieritis Burmeister, 1855); Isonychus sulphureus Mannerheim, 1829 (type species of Isonychus); 
Melolontha rufipes Latreille, 1813 (type species of Sciuropus Dejean, 1833, nomen oblitum, a synonym of Ancistrosoma); Melolontha filitarsis Germar, 1824 (type species of Philochloenia Dejean, 1833); Melolontha subspinosa (type species of Macrodactylus); Philochloenia compacta Erichson, 1847 (type species of Microcrania Burmeister, 1855, a synonym of Barybas); Rhinaspis schrankii Perty, 1833 (a synonym of Rhinaspis aenea (Billberg, 1820), and type species of Rhinaspis); Trichoderma ceylanica Nonfried, 1894 (type species of Trichoderma Nonfried now Junkia).

The following note on the classification of some genera is added to avoid imprecision in Macrodactylini taxonomy. Twenty-two genera have been included in Macrodactylini but were subsequently removed and included in other tribes, and they are commented on below.

Nine genera were included as non-Neotropical members of Macrodactylini (Arrow 1920; Dalla Torre 1913). The Oriental Dichelomorpha Burmeister, 1855, Dicheloschema Moser, 1924, Diphycerus Deyrolle \& Fairmaire, 1878 and Xenoceraspis Arrow, 1920 are now included in Diphycerini (Medvedev 1952; Prokofiev 2015) and another five African or Oriental genera remain incertae sedis (Diphydactylus Thomson, 1858, Hyperius Deyrolle \& Fairmaire, 1878, Photyna Brenske, 1897, Pseudisonychus Frey, 1971 and Metaceraspis Frey, 1962) (Katovich 2008; Prokofiev 2015).

Twelve New World genera were included in Macrodactylini and are now placed in Dichelonychini (Coenonycha Horn, 1876, Dichelonyx and Gymnopyge Linell, 1896), in Diplotaxini (Homalochilus Blanchard, 1850, Homoliogenys Gutiérrez, 1952, Liogenys Guérin-Méneville, 1831 and Pacuvia Curtis, 1845), in Liparetrini (Blepharotoma Blanchard, 1850), in Sericoidini (Apterodemidea Gutiérrez, 1952), in Tanyproctini (Diaphylla Erichson, 1847), or in Phaenomerinae (Oxychirus Quedenfeldt, 1888) (Evans 2003; Katovich 2008; Ruiz-Mazanos 2006). The genus Manonychus Moser, 1919 is placed in Diplotaxini (Gutiérrez 1952, as Liogenyina, Macrodactylini) or remain incertae sedis (Katovich 2008).

A further three genera have been synonymyzed with non-macrodactyline genera: Apterodema Fairmaire, 1884 (a synonym of Sericoides Guérin-Méneville, 1839, Sericoidini), Hilarianus Blanchard, 1850 (a synonym of Liogenys) and Zabacana Saylor, 1946 (a synonym of Epectinaspis Blanchard, 1851, Rutelinae) (Cherman et al. 2016; Martínez 1972; Paucar-Cabrera 2003). The name Epicaulis Dejean nomem nudum (see Bousquet \& Bouchard 2013) was sometimes improperly included in Macrodactylini (e.g., Dalla Torre 1913).

\section{Material and methods}

This study is based on morphological analysis and the terminology used herein follows Beutel \& Lawrence (2005), supplemented by Lawrence et al. (2010). Male genitalia are important diagnostic characters and, whenever possible, illustrations of these structures are added.

Collections with material examined (acronym; curators): The Natural History Museum, London, England (BMNH; Malcolm Kerley, Max Barclay); Muséum national d'Histoire naturelle, Paris, France (MNHN; Antoine Mantilleri, Olivier Montreuil); Museum für Naturkunde, Leibniz-Institut für Evolutionsund Biodiversitätsforschung, Humboldt-Universität zu Berlin, Berlin, Germany (ZMHU; Johannes Frisch, Joachim Willers); Senckenberg Deutsches Entomologisches Institut, Müncheberg, Germany (SDEI; Stephan M. Blank); Museum für Tierkunde, Staatliche Naturhistorische Sammlungen Dresden, Dresden, Germany (SMTD; Olaf Jäger, Klaus-Dieter Klass); Zentralmagazin Naturwissenschaftlicher Sammlungen der Martin-Luther-Universität, Halle, Germany (ZNSM; Karla Schneider). Other collections cited (acronym): Canadian Museum of Nature, Ottawa, Canada (CMNC); Museo Argentino de Ciencias Naturales Bernardino Rivadavia, Bueno Aires, Argentina (MACN); Museo Nacional de Historia Natural, Santiago, Chile (MNNC); Finnish Museum of Natural History, Helsinki, Finland (MNZH); Entomologische Abteilung, Naturhistorisches Museum Basel, Basel, Switzerland (NHMB); 
Naturhistorisches Museum Wien, Vienna, Austria (NHMW); National Museum of Natural History, Prague, Czech Republic (NMPC).

Horn et al. (1990a, b) and Cambefort (2006) were used to locate original type series collections. The date of publication of Blanchard's "Voyage dans l'Amérique méridionale par d'Orbigny" (Blanchard 1835-1847), that of Guérin-Méneville's "Voyage sur la Coquille par L.J. Duperry" (Guérin-Méneville 1830-1831, 1838), that of Dejean's second and third "coleopterous catalogue" (Dejean 1833-1836a, 1836b-1837) and that of "Encyclopédie Méthodique" (LePeletier de Saint-Fargeau \& Audinet-Serville 1828) follow Bousquet (2016). Author names follow the ideas expressed by Dubois (2008).

Primary types labels are quoted as: each label is between square brackets "[ ]", lines separated by a vertical bar "| ", printed parts in bold, and handwritten parts in regular text format.

\section{Results}

Superfamily Scarabaeoidea Latreille, 1802

Family Scarabaeidae Latreille, 1802

Subfamily Melolonthinae Leach, 1819

Tribe Macrodactylini Kirby, 1837

Agaocnemis Moser, 1918

\section{Type species}

Agaocnemis pruina Moser, 1918: 115 (designation: monotypy).

\section{Diagnosis}

Anterior and posterior pronotal margins not beaded (Fig. 2A), posterior margin prominent with a medial tooth (Fig. 2A); prosternum anteriorly concave (similar to Fig. 12K); protibia with three external teeth and without spur (Fig. 2A); male protarsomeres I-II ventrally flattened and with a setal comb (Fig. 2A); elytral striae indistinct, lateral margins beaded, posterior and posterointernal margins not beaded (Fig. 1A); male metatibia internodistally flattened (Fig. 2E); metatarsomere V without internoproximal spine-like setae; abdomen with intersegmental membrane VII-VIII concealed (Fig. 1A).

\section{Remarks}

The genera Agaocnemis, Byrasba, Hamatoplectris and Hieritis have a distinct pronotal posterior lobe bearing a small medial tooth that extends over the scutellum (Fig. 2A-D). Agaocnemis is distinguished as follows (opposition to 1, Byrasba; 2, Hamatoplectris; 3, Hieritis): male metatibia internodistally flattened (Fig. 2E) (1, 3, without flattened area or teeth (Fig. 2F); 2, male metatibia with an internodistal tooth (Fig. 2G)), and female elytron with posterolateral margin broadly beaded (Fig. 1B) (1, 3, female elytral margin finelly beaded; 2, female not known).

Agaocnemis pruina Moser, 1918

Figs 1, 2A, E, 17A-B

Agaocnemis pruina Moser, 1918: 116.

\section{Note}

Agaocnemis pruina was described based on an undetermined number of specimens. 


\section{Material examined}

Lectotype (hereby designated, Fig. 17A-B)

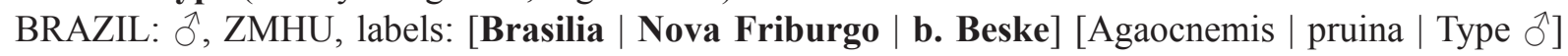
[LECTOTYPE $\mid$ Agaocnemis $\mid$ pruinosa $\mid$ Moser, 1918| $\widehat{\partial} \mid$ des. j. Fuhrmann \& | F.Z. Vaz-de-Mello, 2014].

\section{Paralectotypes $(n=4)$}

BRAZIL: 1 ô, ZMHU, labels: [24154] [“illegible"] [PARALECTOTYPE | Agaocnemis | pruinosa | $\hat{o} \mid$ des. j. Fuhrmann \& | F.Z. Vaz-de-Mello, 2014]; 1 q, ZMHU, labels: [Brasilia | Nova Friburgo | b. Beske] [Agaocnemis | pruina | Type ?] [PARALECTOTYPE | Agaocnemis $\mid$ pruinosa $\mid$ \& $\mid$ des. j. Fuhrmann \& | F.Z. Vaz-de-Mello, 2014]; 1 \%, ZMHU, labels: [Brasilia | Nova Friburgo | b. Beske] [O] [PARALECTOTYPE $\mid$ Agaocnemis $\mid$ pruinosa $|+|$ des. j. Fuhrmann \& | F.Z. Vaz-de-Mello, 2014]; 1 q, ZMHU, labels: [24153] [pruina | Germ, Burm. | Brasil. Germ.] [Agaocnemis Mo | (Liogenys) | pruina Burm. Mos.] [PARALECTOTYPE $\mid$ Agaocnemis $\mid$ pruina $|q|$ des. j. Fuhrmann \& $\mid$ F.Z. Vaz-de-Mello, 2014].

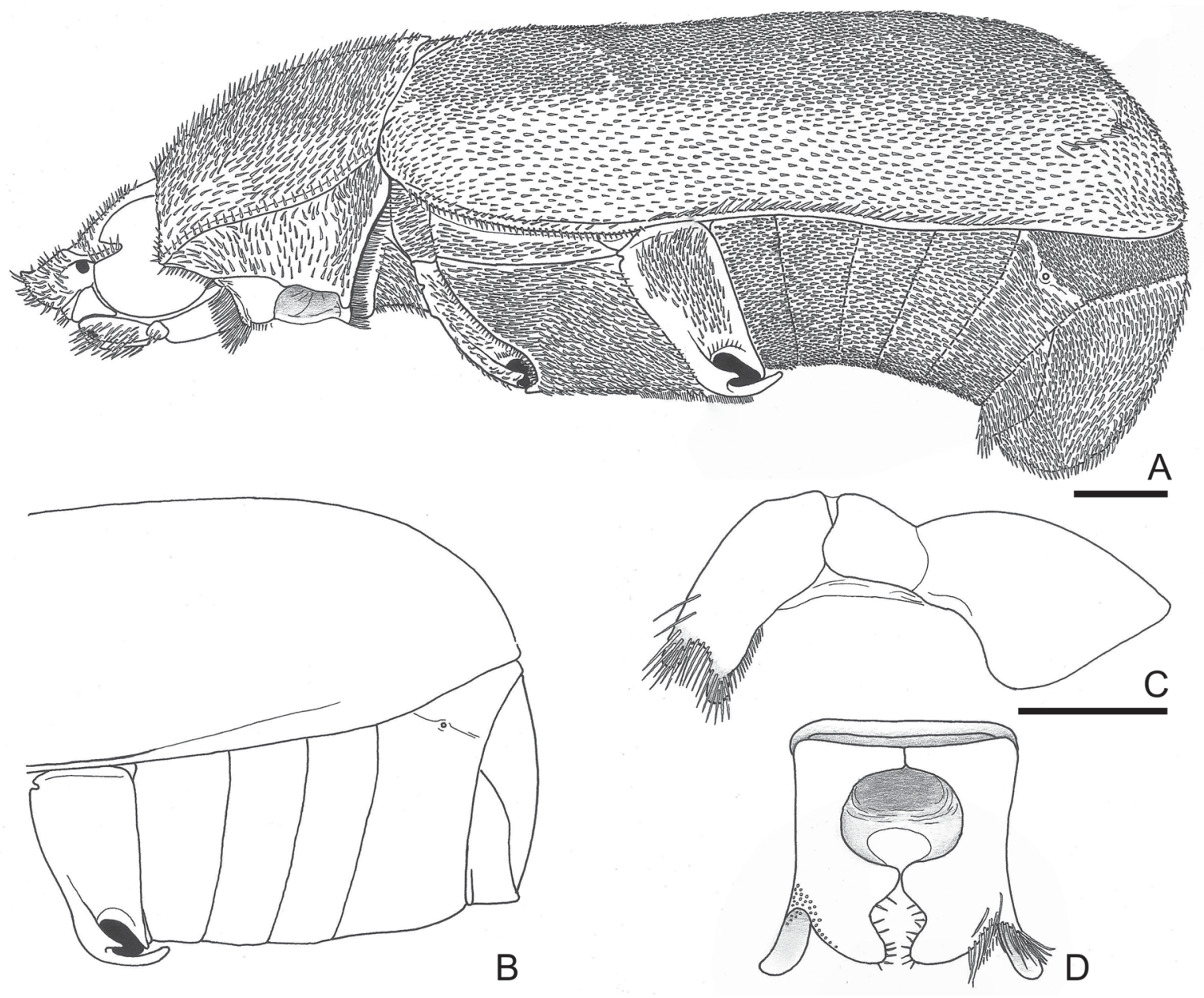

Fig. 1. Agaocnemis pruina Moser, 1918. A. Male habitus, lateral (without some appendages). B. Female abdomen lateral detail. C-D. Aedeagus (lateral, parameres apex). Scale bars $=1 \mathrm{~mm}$. 

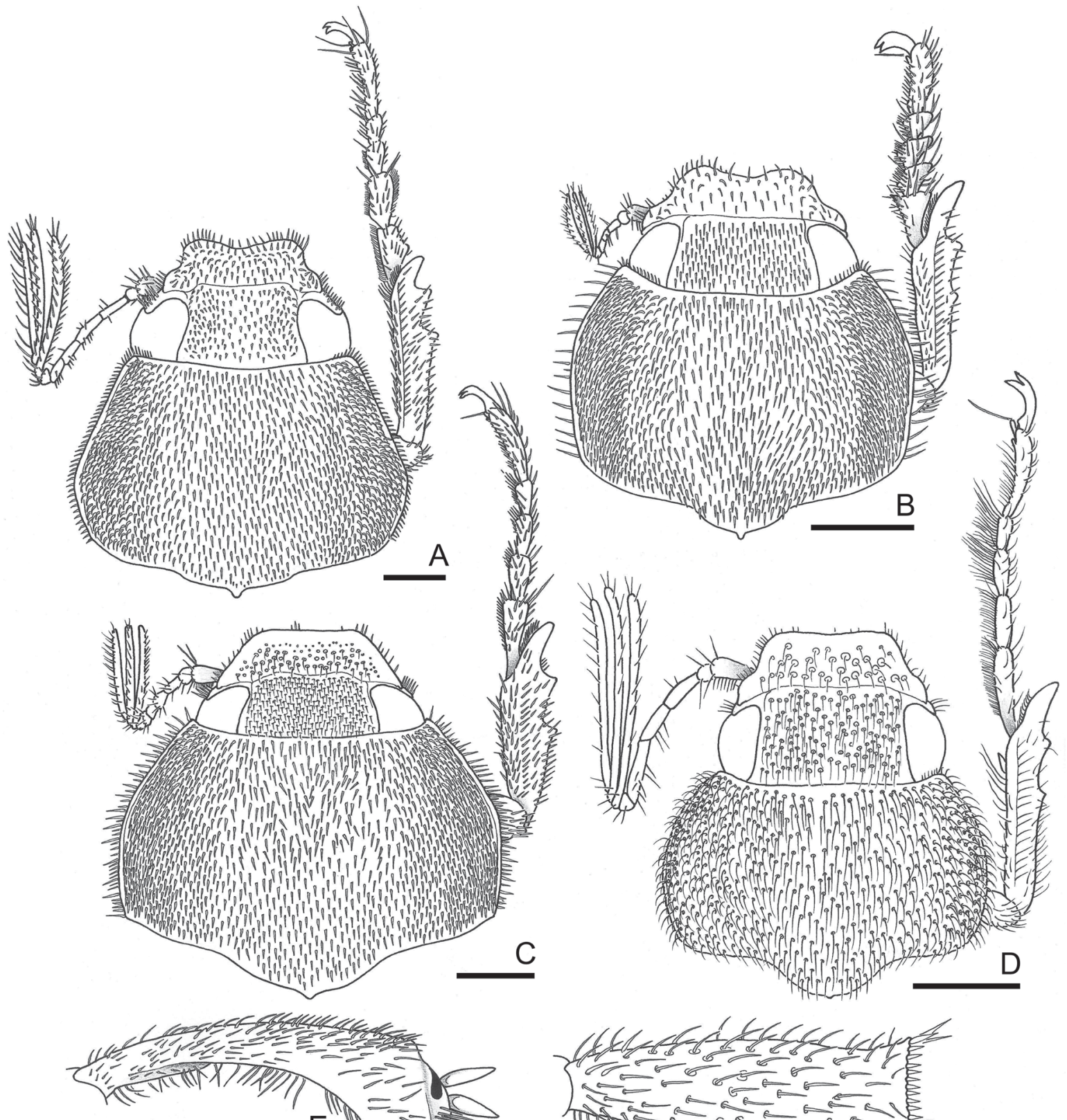

$\mathrm{E}$
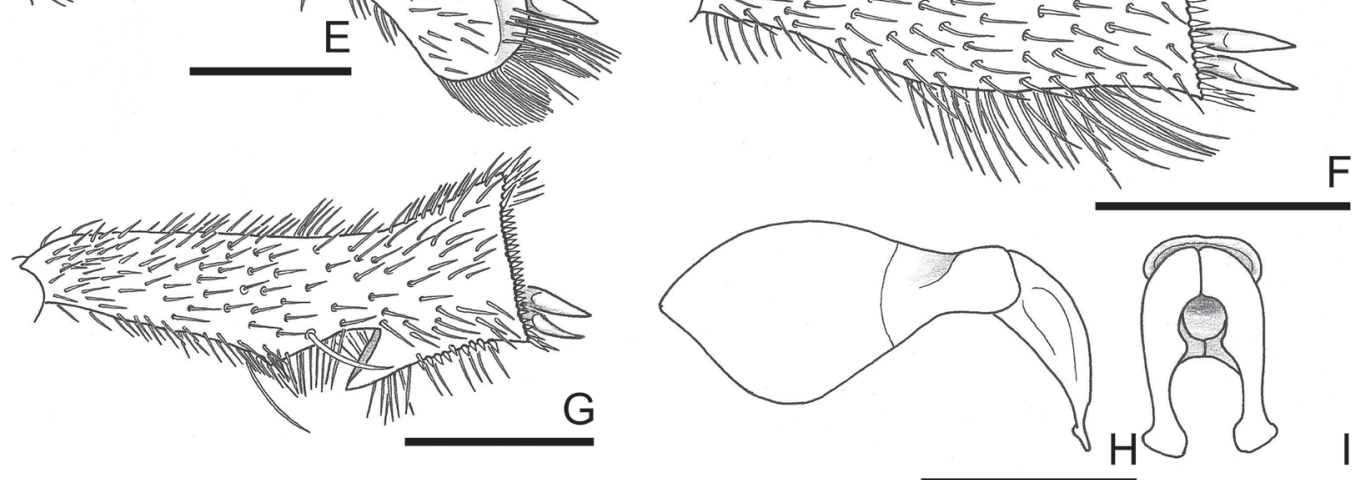

Fig. 2. $\widehat{\jmath}$. A-D. Head-prothorax dorsal, tarsus rotated laterally to apex. E-G. Metatibia. H-I. Aedeagus (lateral, parameres apex). A, E. Agaocnemis pruina Moser, 1918. B, F, H-I. Byrasba volvula (Burmeister, 1855). C, G. Hamatoplectris caracana Frey, 1969. D. Hieritis macrocera Burmeister, 1855. Scale bars = $1 \mathrm{~mm}$. 
Alvarinus Blanchard, 1850

Corminus Burmeister, 1855: 39. Type species: Corminus canescens Burmeister, 1855 (designation: Evans 2003: 254).

Corminus - Katovich 2008: 6 (synonymy).

\section{Type species}

Alvarinus hilarii Blanchard, 1850 (designation: Evans 2003: 224).

\section{Diagnosis}

Clypeal ventral area strongly widened (Fig. 3A); pronotal anterior and posterior margins not beaded (Fig. 3B); prosternum anteriorly concave (similar to Fig. 12K); protibia with two external teeth and without spur (Fig. 3B); elytral striae indistint, posterior and posterointernal margins not beaded; mesoand metafemurs of male with some long and spine-like setae (Fig. 3C); metatarsomere V without spinelike setae; abdomen with intersegmental membrane VII-VIII concealed (Fig. 3A).

\section{Alvarinus canescens (Burmeister, 1855)}

Figs 3, 17C-D

Corminus canescens Burmeister, 1855: 40.

Alvarinus canescens - Katovich 2008: 6 (Corminus synonym of Alvarinus).

\section{Note}

Corminus canescens was described based on an undetermined number of males and females, and at least one specimen of each sex. Only one male was found and designated as lectotype.

\section{Material examined}

Lectotype (hereby designated, Fig. 17C-D)

BRAZIL: Ô, ZNSM, labels: [Corminus | Burm.] \{drawer label\} [canescens | Germ. | Bras. Br.] \{drawer label\} [Nov. | Frib.] [LECTOTYPE ô | Corminus canescens | Burmeister, 1855 | des. J. Fuhrmann \& | F.Z. Vaz-de-Mello, 2014].

\section{Ancistrosoma Curtis, 1835, nomen protectum}

Sciuropus Dejean, 1833: 162, nomen oblitum. Type species: Melolontha rufipes Latreille, 1813 (designation: monotypy).

\section{Type species}

Ancistrosoma klugii Curtis, 1835 (designation: monotypy).

\section{Remark}

As stated by Bousquet \& Bouchard (2013) Sciuropus is a senior synonym of Ancistrosoma; the latter name, however, was used as valid for a long time (Guimarães 1977; Ayquipa \& Cueva 1979; AguilarFernández 1980; Valdivieso-Jara \& Núñez-Sacarías 1984; Cisneros 1995; Peña \& Bennet 1995; RestrepoGiraldo \& López-Ávila 2000; Rogg 2000; Bentley \& Vilca 2001; Peck et al. 2002; Morales-Valles et al. 2003; Evans 2003; Evans \& Smith 2005, 2007, 2009; Egúsquiza-Bayona et al. 2006; Katovich 2008, 2011; Mera-Velasco et al. 2010; Santandeu 2010; Valoy et al. 2011, 2015; Neita-Moreno et al. 2012; 
Rojo-Jiménez 2014; López-García et al. 2015; Ratcliffe et al. 2015) (Ancistrosoma - Gess \& Gess 2014 lapsus calami of Ancistromma Fox, 1894, Hymenoptera), and Sciuropus was used only once since its publication (Rühl 1888). The present study proposes the reversal of precedence of Ancistrosoma, nomen protectum over Sciuropus, nomen oblitum in accordance with ICZN (1999) Article 23.9.

\section{Anomonyx Saylor, 1940}

Anomalonyx Moser, 1921: 156 (non Weise, 1903).

Anomonyx Saylor, 1940: 46 (replacement name for Anomalonyx).

\section{Type species}

Anomalonyx uruguayensis Moser, 1921 (designation: monotypy).

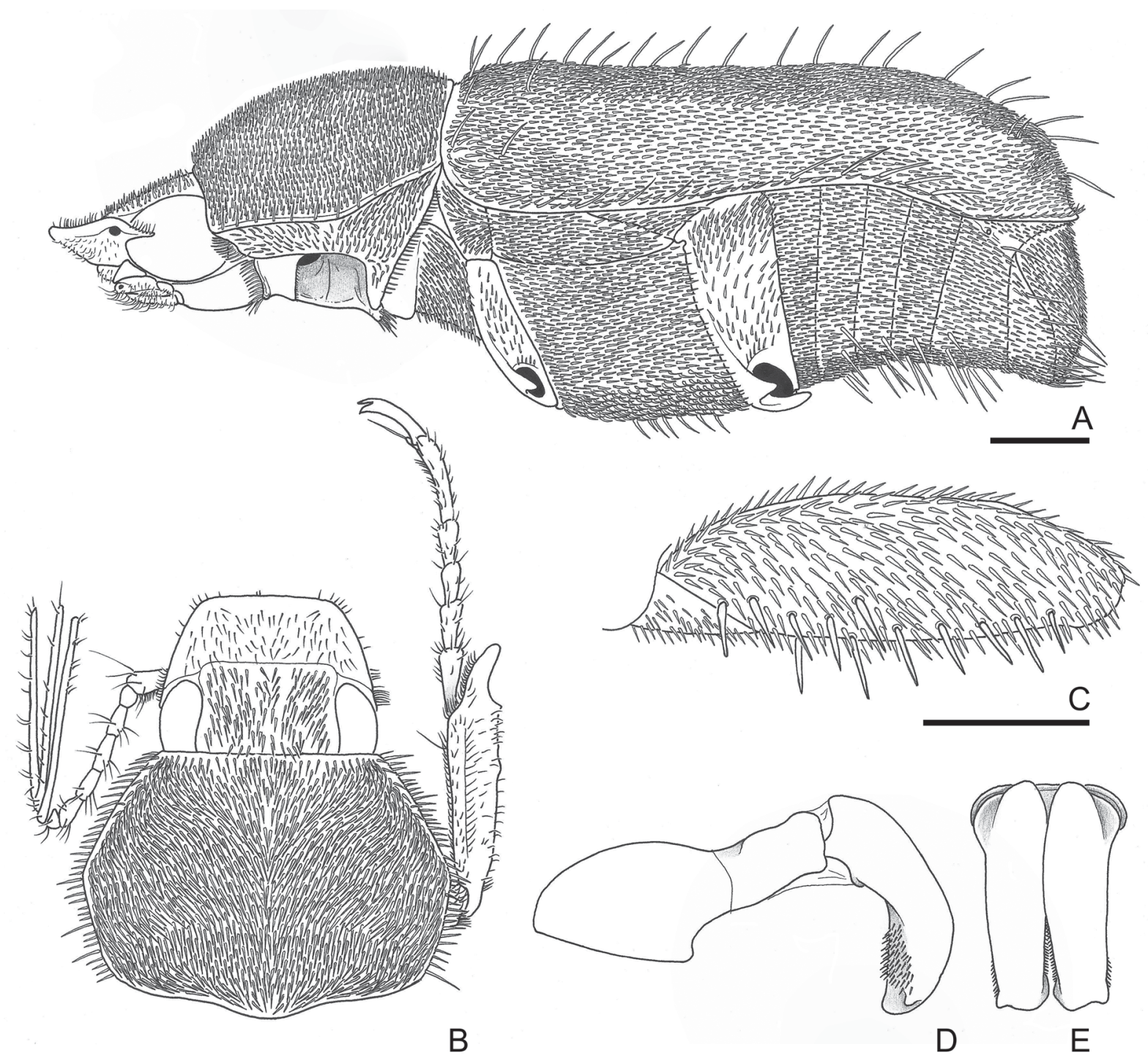

Fig. 3. Alvarinus canescens Burmeister, 1855, $\widehat{\jmath}$. A. Habitus, lateral (without some appendages). B. Head-prothorax dorsal, tarsus rotated laterally to apex. C. Metafemur. D-E. Aedeagus (lateral, parameres apex). Scale bars $=1 \mathrm{~mm}$. 


\section{Diagnosis}

Clypeal ventral area large and vertically deflected; labrum slightly emarginate; pronotal anterior and posterior margins not beaded; prosternum with an anterior longitudinal carina; elytral striae indistinct, posterior margin not beaded and posterointernal margin beaded; metatarsomere I as long as II+III (Fig. 4B); metatarsomere $\mathrm{V}$ with internoproximal spine-like setae (Fig. 4B).

\section{Remarks}

The genera Anomonyx, Oedichira Burmeister, 1855 and Plectris LePeletier de Saint-Fargeau \& AudinetServille, 1828 are similar to each other. Anomonyx is distinguished as follows (opposition to 1, Oedichira; 2, Plectris): clypeal ventral area vertically deflected (1, as Anomonyx; 2, vertically deflected or not); metatarsomere I as long as II+III $(1,2$, as long as II-IV); metatarsomere II at least twice as long as wide (1, wider than long; 2, as Anomonyx). Future cladistic analyses and a taxonomic review of Plectris including Anomonyx and Oedichira are needed to determine the possible synonymy between these genera.

Anomonyx uruguayensis (Moser, 1921)

Figs 4B, E-F, 17E-F

Anomalonyx uruguayensis Moser, 1921: 156.

Anomonyx uruguayensis Saylor, 1940: 46.

Note

Anomalonyx uruguayensis was described based on an undetermined number of specimens.

\section{Material examined}

Lectotype (hereby designated, Fig. 17E-F)

URUGUAY: §̃, ZMHU, labels: [Uruguay | Dolores | Breuer] [Anomalonyx | uruguayensis | Type Mos]

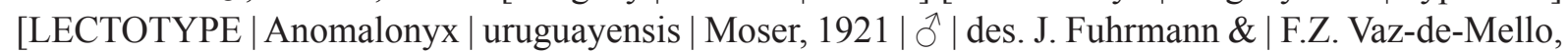
2014] [Anomonyx | uruguayensis $\mid$ (Moser, 1921) $|\widehat{\partial}|$ det. J. Fuhrmann \& | F.Z. Vaz-de-Mello, 2014].

\section{Paralectotypes $(n=4)$}

URUGUAY: $1 \lambda$, ZMHU, labels: [Uruguay | Dolores | Breuer] [PARALECTOTYPE | Anomalonyx | uruguayensis $\mid$ Moser, $1921|\widehat{\partial}|$ des. J. Fuhrmann \& | F.Z. Vaz-de-Mello, 2014]; 1 ふ, ZMHU, labels: [Uruguay | Dolores | Breuer] [Anomalonyx Mos |n. gen. "illegible" Philochlaenia | uruguayensis Mos n. sp.] [PARALECTOTYPE | Anomalonyx | uruguayensis | Moser, 1921 $|\hat{\sigma}|$ des. J. Fuhrmann \& $\mid$ F.Z. Vaz-de-Mello, 2014]; 1 Jे, ZMHU, labels: [Uruguay | Dolores | Breuer] [Philogenis | "illegible" genus | (nov. ?)] [Anomolonyx | uruguayensis | Moser | fide K. Katovich 02] [PARALECTOTYPE | Anomalonyx | uruguayensis | Moser, $1921|\hat{\jmath}|$ des. J. Fuhrmann \& | F.Z. Vaz-de-Mello, 2014]; 1 ठ, ZMHU, labels: [Uruguay | Dolores | Breuer] [Anomolonyx | uruguayensis | Moser | fide K. Katovich 02] [PARALECTOTYPE | Anomalonyx | uruguayensis | Moser, 1921 $\mid$ ठै $\mid$ des. J. Fuhrmann \& $\mid$ F.Z. Vaz-de-Mello, 2014].

\section{Barybas Blanchard, 1850}

Ctilocephala Burmeister, 1855: 78. Type species: Ctilocephala pellucens Burmeister, 1855 (original designation).

Eubarybas Gutiérez, 1952: 216. Type species: Eubarybas asper Gutiérez, 1952 (original designation). Hercitis Burmeister, 1855: 79, syn. nov. Type species: Hercitis pygmaea Burmeister, 1855 (designation: monotypy), syn. nov. of Barybas nana. 
Microcrania Burmeister, 1855: 75, 536 (synonymy). Type species: Philochloenia compacta Erichson, 1847 (designation: Evans 2003: 230).

Pseudohersitis Moser, 1921: 174. Type species: Pseudohersitis viridiaenea Moser, 1921 (designation: Evans 2003: 343).

Ctilocephala - Katovich 2008: 6 (synonymy).

Eubarybas - Katovich 2008: 6 (synonymy).

Pseudohersitis - Katovich 2008: 24 (synonymy).

\section{Type species}

Barybas nana Blanchard, 1850 (designation: Bates 1887: 150).

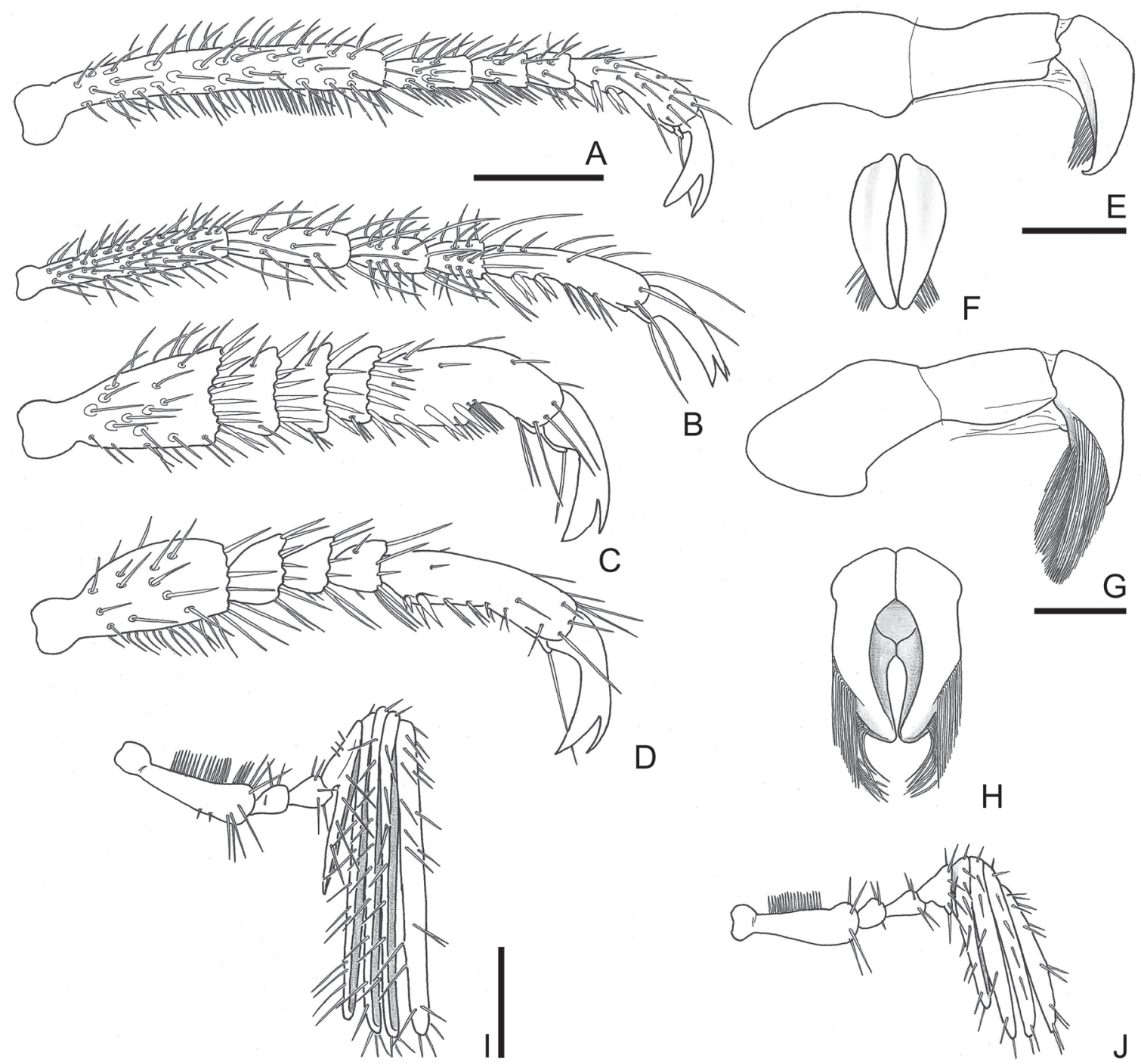

Fig. 4. A-C. Male metatarsus. D. Female metatarsus. E-H. Aedeagus (lateral, parameres apex). I-J. Antenna $(\hat{\partial},+$ ) $)$. A. Plectris tomentosa LePeletier de Saint-Fargeau \& Audinet-Serville, 1828. B, E-F. Anomonyx uruguayensis Moser, 1921. C-D, G-J. Oedichira pachydactyla Burmeister, 1855. Scale bars $=1 \mathrm{~mm}$. 


\section{Diagnosis}

Clypeus transverse and narrow (Fig. 5B), or trapezoid (Fig. 5A), clypeal posterior angle partially covering the canthus (Fig. 5A-B), ventral area deflected vertically or not (Fig. 5C-H); pronotal anterior and posterior margins not beaded, lateral margins crenulate (Fig. 5A-B); prosternum anteriorly concave (similar to Fig. 12K); mesoscutum-scutellum limit angulate; mesoscutum with a transverse carina (Fig. 6G); male protarsomere I with an internodistal tooth (Fig. 5A-B); elytral striae indistinct, posterior margin not beaded, posterointernal margin beaded; metatarsomere $\mathrm{V}$ with internoproximal spine-like setae.

\section{Remarks}

Burmeister (1855) described Ctilocephala, Hercitis and Microcrania, and placed them in the Microcranidae division (these genera were differentiated from each other by antennal and tarsal claw characters). The author (Burmeister 1855) also proposed the synonymy of Microcrania with Barybas Blanchard (as an invalid name), described Barybas Burmeister, and noted that Barybas nana (type species of Barybas Blanchard) was probably a synonym of Hercitis pygmaea (type species of Hercitis). Harold (1869) proposed the replacement name Byrasba for Barybas Burmeister, and Bates (1887) gave precedence to Barybas Blanchard over Microcrania. Moser (1921) described Pseudohercitis as a similar genus to those placed in Microcranidae, and discussed the differences in antennae and tarsal claws of these genera. Gutiérrez (1952), also using antennal and tarsal claw characters as diagnoses, described Eubarybas Gutiérrez, 1952, and erected the subtribe Barybasina for Eubarybas and those genera formerly in Microcranidae. Katovich (2008) proposed Pseudohercitis and Eubarybas as synonyms of Barybas Blanchard.

The lectotype of Barybas nana, photos of the syntype of H. pygmaea (by Holger Dombrow), two specimens of B. nana from the Fry collection (BMNH, with in litteris note about B. nana $=H$. pygmaea), and the type series of Hercitis zikani Moser, 1921 (ZMHU) were studied, and the synonymy of Hercitis pygmaea with Barybas nana is proposed here. The tarsal and antennal variation that were presented as the basis of generic diagnoses in previous studies, are also present in other Macrodactylini genera (e.g., Ceraspis; Dicrania LePeletier de Saint-Fargeau \& Audinet-Serville, 1828; Faula Blanchard, 1850; Plectris), and are noted here as interspecific variation. The other three species previously placed in Hercitis are transferred to Barybas, which now comprises 53 species of which the following three are new combinations: Barybas flavosquamosa (Moser, 1924) comb. nov.; Barybas testacea (Moser, 1918) comb. nov.; Barybas zikani (Moser, 1921) comb. nov.

Colporhina is a monotypic genus (C. bifoveolata, a junior synonym of Melolontha variegatus Germar, 1824) which has been synonymized twice: with Barybas (Lacordaire 1856) and with Isonychus (Gemminger \& Harold 1869). Blanchard (1850: 94) placed Melolontha variegatus Germar, 1824 in Barybas and Burmeister (1855: 47) placed the species in Isonychus and noted the similarity between Colpohrina and Micorcrania and Hercitis (both synonyms of Barybas). After that, Lacordaire (1856) proposed $C$. bifoveolata as a synonym of $M$. variegatus, and confirmed the species position within Barybas (in agreement with Blanchard 1850). Gemminger \& Harold (1869) confirmed this synonymy but placed the species in Isonychus (in agreement with Burmeister 1855). The type series of C. biflaveolata was not found, and the possibility that Colporhina might be a senior synonym of Barybas needs to be checked.

Barybas nana Blanchard, 1850

Figs $5 \mathrm{~A}, \mathrm{C}-\mathrm{D}, 6 \mathrm{G}, 17 \mathrm{G}-\mathrm{H}, 21 \mathrm{G}-\mathrm{K}$

Barybas nanus Blanchard, 1850: 94.

Hercitis pygmaea Burmesiter, 1855: 79, syn. nov.

Barybas nana-Blackwelder 1944: 233 (name emendation). 


\section{Note}

Barybas nana and Hercitis pygmaea were described based on an undetermined number of specimens.

\section{Material examined}

Lectotype (here designated, Fig. 17G-H)

BRAZIL: $\widehat{\jmath}$, MNHN, labels: [Capte. | des mines] [MUSÉUM PARIS | Ouest Capte | des Mises] [B. nanus | Cat. Mus. | Brésil. | M.A. de St. Hilaire] [LECTOTYPE | Barybas nanus | Blanchard, 1850 | des. J. Fuhrmann \& | F.Z. Vaz-de-Mello, 2014].

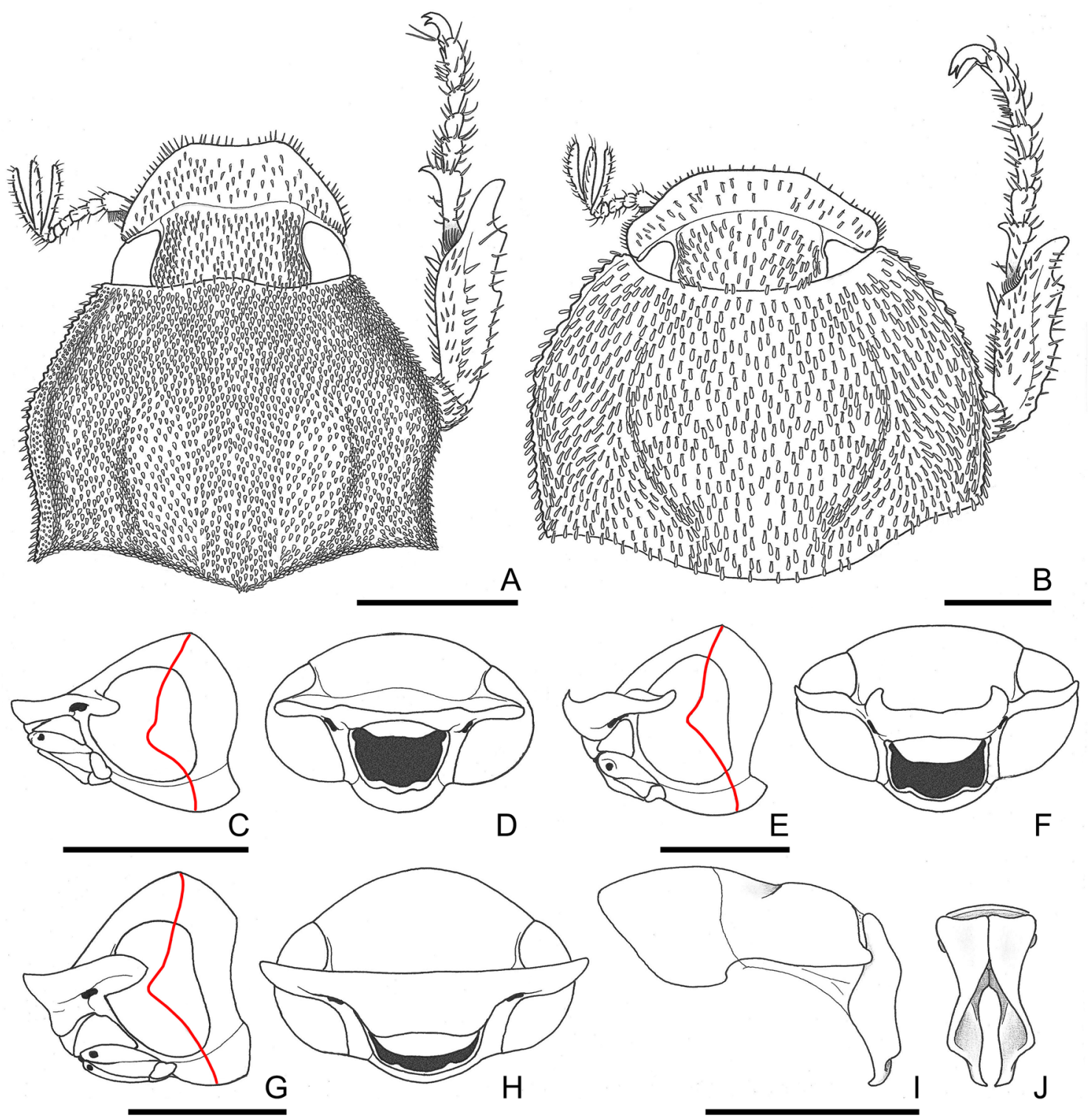

Fig. 5. Barybas Blanchard, 1850. A-B. Head-prothorax dorsal, tarsus rotated laterally to apex. C-H. Male head (lateral, frontal), line = prothorax limit. I-J. Aedeagus (lateral, parameres apex). A, C-D. Barybas nana Blanchard, 1850. B, G-J. Barybas viridiaenea Moser, 1921. E-F. Barybas pellucens Burmeister, 1855. Scale bars $=1 \mathrm{~mm}$. 


\section{Paralectotype}

BRAZIL: $\hat{\jmath}$, MNHN, labels: [Capte. | des mines] [MUSÉUM PARIS | Ouest Capte | des Mises] [PARALECTOTYPE $\mid$ Barybas nanus $\mid$ Blanchard, $1850|\widehat{\jmath}|$ des. J. Fuhrmann \& $\mid$ F.Z. Vaz-de-Mello, 2014].

Syntype of Hercitis pygmaea (Fig. 21G-K)

BRAZIL: $\widehat{\jmath}$, ZNSM, labels: $\widehat{\jmath}$, [Hercitis | Burm.] \{drawer label\} [pygmaea | Burm.| Bras. Br.] \{drawer label\}.

\section{Remarks}

The present study proposes the synonymy of H. pygmaea (type species of Hercitis) with B. nana (type species of Barybas Blanchard). See generic remarks above for historical and taxonomic comments. The syntype of H. pygmaea is being studied by Holger Dombrow (pers. comm.).

Barybas pellucens (Burmeister, 1855)

Figs 5E-F, 17I-J

Ctilocephala pellucens Burmeister, 1855: 78.

Barybas pellucens - Katovich 2008: 6 (Ctilocephala synonym of Barybas).

\section{Note}

Ctilocephala pellucens was described based on an undetermined number of males and females.

\section{Material examined}

Lectotype (here designated, Fig. 17I-J)

COLOMBIA: $\hat{\partial}$, ZNSM, labels: [Ctilocephala |Burm.] \{drawer label\} [pellucens | Phil. p. \{illegible\} | biguttata Dj. | Columb.] \{drawer label\} [LECTOTYPE | Ctilocephala | pelluscens | Burmeister, $1855 \mid{ }^{\lambda}$ | des. J. Fuhrmann \& | F.Z. Vaz-de-Mello, 2014].

Paralectotypes $(\mathrm{n}=2)$

COLOMBIA: 1 ô, ZNSM, labels: [Ctilocephala |Burm.] \{drawer label\} [pellucens | Phil. p. \{illegible\} | biguttata Dj. |Columb.] \{drawer label\} [PARALECTOTYPE $\mid$ Ctilocephala|pelluscens $\mid$ Burmeister, 1855 $\left|\hat{O}^{\hat{N}}\right|$ des. J. Fuhrmann \& | F.Z. Vaz-de-Mello, 2014]; 1 + , MNHN, labels: [Ctilocephala | Burm.] \{drawer label\} [pellucens | Phil. p. \{illegible\} | biguttata Dj. | Columb.] \{drawer label\} [PARALECTOTYPE | Ctilocephala $\mid$ pelluscens $\mid$ Burmeister, $1855 \mid$ o $\mid$ des. J. Fuhrmann \& $\mid$ F.Z. Vaz-de-Mello, 2014].

\section{Barybas viridiaenea (Moser, 1921)}

Figs $5 \mathrm{~B}, \mathrm{G}-\mathrm{J}, 17 \mathrm{~K}-\mathrm{L}$

Pseudohercitis viridiaenea Moser, 1921: 174.

Barybas viridiaenea - Katovich 2008: 6 (Pseudohercitis synonym of Barybas).

Note

Pseudohercitis viridiaenea was described based on an undetermined number of males. 


\section{Material examined}

Lectotype (here designated, Fig. 17K-L)

BRAZIL: ${ }^{\lambda}$, ZMHU, labels: [Corumba | Matt Grosso] [Pseudohercitis | viridiaenea | Type Mos] [LECTOTYPE | Pseudohercitis | viridiaenea $\mid$ Moser, $1921 \mid$ ô $\mid$ des. J. Fuhrmann \& | F.Z. Vaz-de-Mello, 2014].

\section{Byrasba Harold, 1869, revalidated}

Barybas Burmeister, 1855: 20 (non Blanchard 1850).

Byrasba Harold, 1869: 122 (replacement name for Barybas Burmeister).

Byrasba - Katovich 2008: 6 (synonymy with Rhinaspis Perty, 1833).

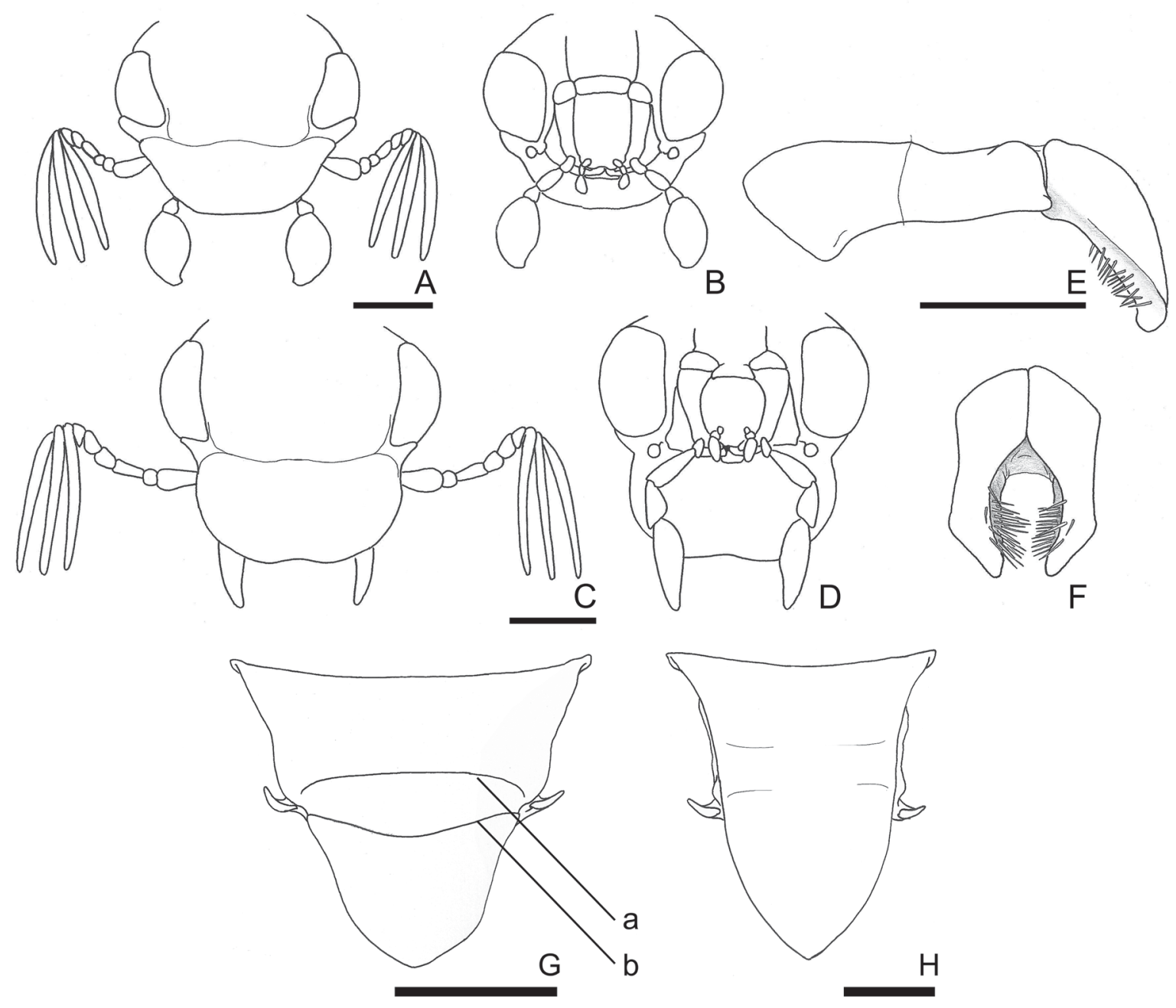

Fig. 6. $\hat{o}$. A-D. Head (dorsal, ventral). E-F. Aedeagus (lateral, parameres apex). G-H. Mesoalinotum. A-B, E-F. Clavipalpus dejeani Laporte, 1832. C-D. Paulosawaya ursina (Blanchard, 1850) comb. nov. G. Barybas nana Blanchard, 1850. H. Macrodactylus pumilio Burmeister, 1855. a = scutum transverse carina; $b=$ scutum-scutellum limit angulate. Scale bars $=1 \mathrm{~mm}$. 


\section{Type species}

Barybas volvulus Burmeister, 1855 (designation: monotypy).

\section{Diagnosis}

Pronotal anterior and posterior margins not beaded (Fig. 2B), posterior margin prominent with a medial tooth (Fig. 2B); prosternum anteriorly concave (similar to Fig. 12K); protibia with three external teeth and without spur (Fig. 2B); male protarsomere I with an internomedial tooth and II-VI with external projection (Fig. 2B); elytron with five longitudinal grooves between internal margin and humerus, lateral margins beaded, posterior and posterointernal margins not beaded; metatarsomere $\mathrm{V}$ without internoproximal spine-like setae; abdomen with intersegmental membrane VII-VIII concealed.

\section{Remarks}

See Agaocnemis. The present study revalidates Byrasba, formerly a synonym of Rhinaspis, based on the following characters (opposition to Rhinaspis): pronotal lateral margins straight (Fig. 2B) (crenulate, Fig. 10D-F); posterior margin prominent with a medial tooth (Fig. 2B) (posterior margin not prominent and unarmed, Fig. 10D-F); protibial spur absent (present); protarsomeres II-IV with externodistal projection (without projection); elytron with five weakly prominent lines between internal margin and humerus (with three lines).

Byrasba volvula (Burmeister, 1855)

Figs 2B, F, H-I, 17M-N

Barybas volvulus Burmeister, 1855: 20.

Byrasba volvula - Harold, 1869: 1142.

Rhinaspis volvula - Katovich, 2008: 6 (Byrasba synonym of Rhinaspis).

\section{Note}

Barybas volvulus was described based on an undetermined number of males and females.

\section{Material examined}

Lectotype (hereby designated, Fig. 17M-N)

BRAZIL: ${ }^{\lambda}$, ZNSM, labels: [Barybas | Burm] \{drawer label\} [volvulus | Bras. Frey.] \{drawer label\} [Are- $\mid$ as] [LECTOTYPE | Barybas volvulus | Burmeister, $1855 \mid$ ठ | des. J. Fuhrmann \& | F.Z. Vaz-deMello, 2014] [Byrasba volvulus | (Burmeister, 1855) | det. J. Fuhrmann | 2014 §]].

\section{Paralectotypes $(n=2)$}

BRAZIL: 1 ก, ZNSM, labels: [Barybas | Burm] \{drawer label\} [volvulus | Bras. Frey.] \{drawer label\} [6995] [Byrasba | volvulus | (Burmeister) | Det. K. Katovich 2002] [PARALECTOTYPE | Barybas volvulus | Burmeister, $1855|\hat{o}|$ des. J. Fuhrmann \& | F.Z. Vaz-de-Mello, 2014]; 1 , ZNSM, labels: [Barybas $\mid$ Burm] \{drawer label\} [volvulus | Bras. Frey.] \{drawer label\} [PARALECTOTYPE | Barybas volvulus | Burmeister, 1855 | + | des. J. Fuhrmann \& | F.Z. Vaz-de-Mello, 2014].

\section{Type species}

Calodactylus Blanchard, 1850

Calodactylus tibialis Blanchard, 1850 (designation: monotypy). 


\section{Diagnosis}

Prosternum with an anterior longitudinal carina; protibia with two teeth and with a spur; meso- and metatibiae medially enlarged (Fig. 7E); meso- and metatarsal claws simple; male ventrite VI with two parallel and setose carina (Fig. 7B).

\section{Remarks}

The genera Calodactylus and Dasyus LePeletier de Saint-Fargeau \& Audinet-Serville, 1828 are similar to each other. They can be distinguished by enlargements of the meso- and metatibiae, Calodactylus have a medial enlargement whereas Dasyus have medial and distal areas of the same width. An evident characteristic of these genera is the simple meso- and metatarsal claws, which also occurs in male Pectinosoma Arrow,1913 and some species of Ancistrosoma, Barybas, Ceraspis and Faula.

Calodactylus tibialis Blanchard, 1850

Figs 7, 17O-P

Calodactylus tibialis Blanchard, 1850: 91.

\section{Note}

Calodactylus tibialis was described based on an undetermined number of specimens.

\section{Material examined}

Lectotype (here designated, Fig. 17O-P)

BRAZIL: ${ }^{\lambda}$, MNHN, labels: [11 | 44] [MUSÉUM PARIS | Rio | de Castelnau] [Calodactylus tibialis, Bl.] [LECTOTYPE $\hat{\sigma} \mid$ Calodactylus tibialis $|\hat{\sigma}|$ Blanchard, $1850 \mid$ des. J. Fuhrmann \& $\mid$ F.Z. Vaz-deMello, 2014].

\section{Paralectotype}

BRAZIL: ð̊, MNHN, labels: [11 | 44] [MUSÉUM PARIS | Rio | de Castelnau] [PARALECTOTYPE ð | Calodactylus tibialis | ठ̂ | Blanchard, 1850 | des. J. Fuhrmann \& | F.Z. Vaz-de-Mello, 2014].

Ceraspis LePeletier de Saint-Fargeau \& Audinet-Serville, 1828

\section{Type species}

Ceraspis pruinosa LePeletier de Saint-Fargeau \& Audinet-Serville, 1828 (designation: Lacordaire 1856: 252); a synonym of C. bivulnerata (Germar, 1824).

\section{Diagnosis}

Body covered by scale-like or stout setae; pronotal anterior margin not beaded; pronotum-scutellum contact acutely sinuous (Fig. 8C); prosternum with an anterior longitudinal carina; protibia with two external teeth and without spur; meso- and metacoxae contiguous; metatarsomere $\mathrm{V}$ with internoproximal spine-like setae.

\section{Remarks}

Ancistrosoma, Ceraspis, Chariodema, Faula, Manopus Conte de Castelnau, 1840, Pectinosoma and Pseudopectinosoma have a sinuous pronotum-scutellum contact (Fig. 8A-E). Ceraspis is distinguished by the medially contiguous meso- and metacoxae (separate in other genera). 
The present study revalidates Faula, formerly a synonym of Ceraspis. Consequently 27 species are transferred to Faula, and Ceraspis now comprises 75 species: C. ajonjoli Mora-Aguilar, Delgado, and Vallejo, 2013, C. albipennis Frey, 1973, C. albovaria Frey, 1962, C. alvarengai Frey, 1973, C. amazonica Frey, 1962, C. amoema Frey, 1962, C. bivulnerata, C. brittoni Frey, 1962, C. bufo Frey, 1962, C. burmeisteri Frey, 1962, C. castaneipennis Blanchard, 1850, C. cinerea Moser, 1921, C. citrina Blanchard, 1850, C. colon Burmeister, 1855, C. conspersa Burmeister, 1855, C. convexicollis Frey, 1969, C. costulata Frey, 1965, C. decora Gory, 1831, C. diversa Frey, 1962, C. dorsata Burmeister, 1855, C. dorsipicta Frey, 1972, C. duckei Ohaus, 1911 (type species of monotypic subgenus Isoceraspis Ohaus, 1911), C. elegans Nonfried, 1892, C. elongata Frey, 1962, C. farinosa Burmeister, 1855, C. femorata Frey, 1969, C. flava Blanchard, 1850, C. fulva Blanchard, 1850, C. gibbicollis Blanchard, 1850, C. globicollis Frey, 1962, C. griseosquamosa Moser, 1921, C. guttata Blanchard, 1850, C. imitatrix Nonfried, 1892, C. kuntzeni Moser, 1921, C. lepida Frey, 1973, C. leucosoma Blanchard, 1850, C. linharensis Frey, 1973, C. lurida Frey, 1962, C. martinezi Frey, 1962, C. melanoleuca LePeletier de Saint-Fargeau \& AudinetServille, 1828, C. mixta Blanchard, 1850, C. moseri Frey, 1962, C. mustela Frey, 1962, C. mutica

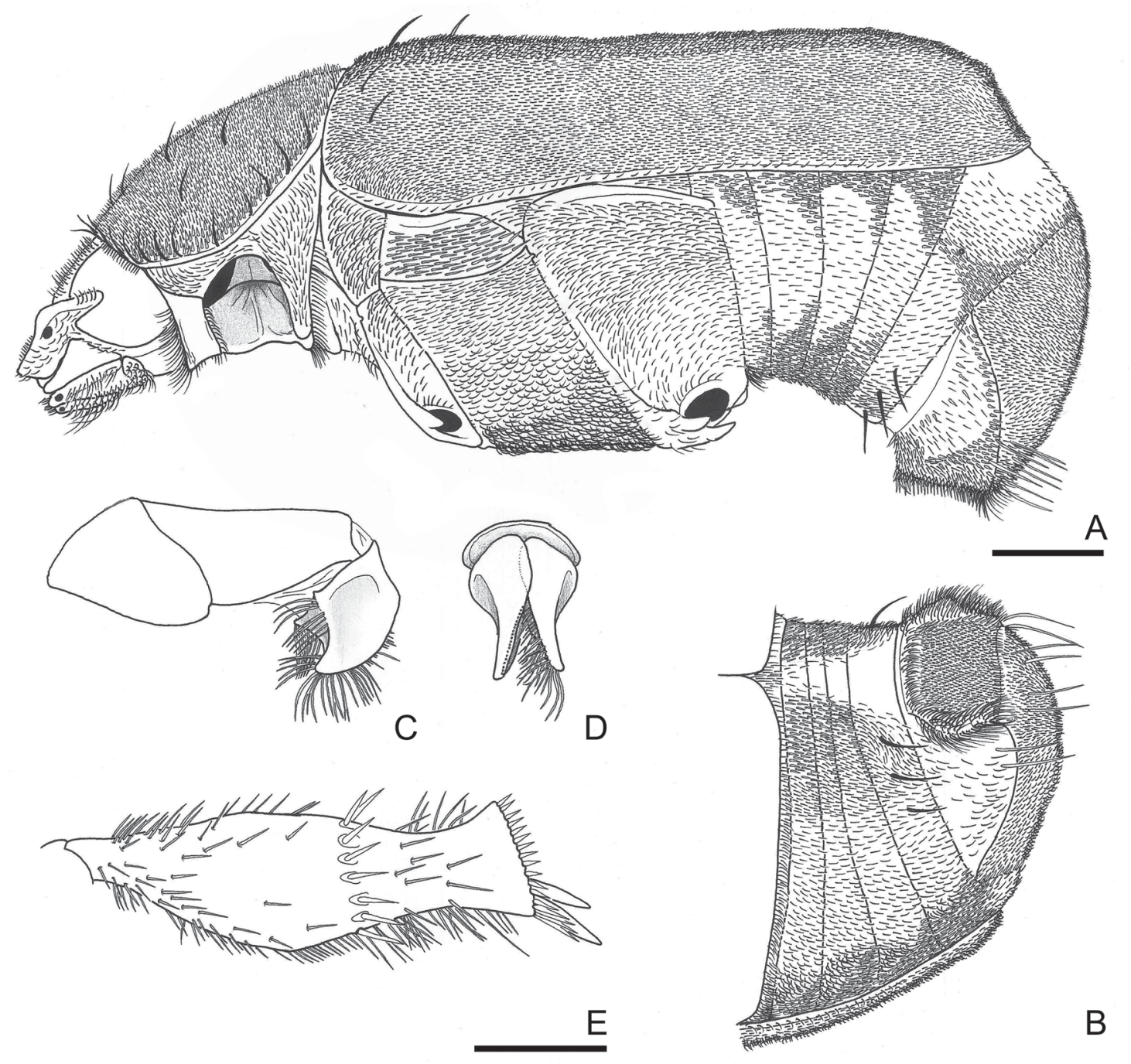

Fig. 7. Calodactylus tibialis Blanchard, 1850. A. Male habitus, lateral (without some appendages). B. Male abdomen lateroventral detail. C-D. Aedeagus (lateral, parameres apex). E. Metatibia. Scale bars $=1 \mathrm{~mm}$. 
Moser, 1921, C. nitida Frey, 1962, C. nivea LePeletier de Saint-Fargeau \& Audinet-Serville, 1828, C. niveipennis Gistel, 1857, C. oblonga Moser, 1919, C. obscura Blanchard, 1850, C. ocellata Frey, 1962, C. ornata Frey, 1962, C. pallida Blanchard, 1850, C. pauperata Burmeister, 1855, C. penai Frey, 1964, C. pereirae Frey, 1962, C. plaumanni Frey, 1962, C. pulchra Frey, 1962, C. rotundicollis Frey, 1973, C. ruficollis Frey, 1962, C. setiventris Moser, 1921, C. signata Blanchard, 1850, C. sparsesetosa Frey, 1972, C. squamulata Moser, 1924, C. striata Frey, 1973, C. subvittata Moser, 1921, C. sulcicollis Moser, 1921, C. tenuisquamosa Frey, 1962, C. tibialis Blanchard, 1850, C. unguicularis Moser, 1919, C. variegata Perty, 1833, C. ventralis Frey, 1862, C. vestita Blanchard, 1850, C. vittata Moser, 1919, C. vulpes Frey, 1962 and C. zikani Moser, 1924.

Ceraspis bivulnerata (Germar, 1824)

Figs $8 \mathrm{C}, 9 \mathrm{~F}-\mathrm{G}, 18 \mathrm{~A}-\mathrm{B}$

Melolontha bivulnerata Germar, 1824: 1824.

Ceraspis pruinosa LePeletier de Saint-Fargeau \& Audinet-Serville, 1828: 371.

Ceraspis albida Guérin-Méneville, 1831: fig. 24bis.

Ceraspis bivulnerata - Burmeister 1855: 93.

Ceraspis pruinosa - Blanchard 1850: 92 (synonymy).
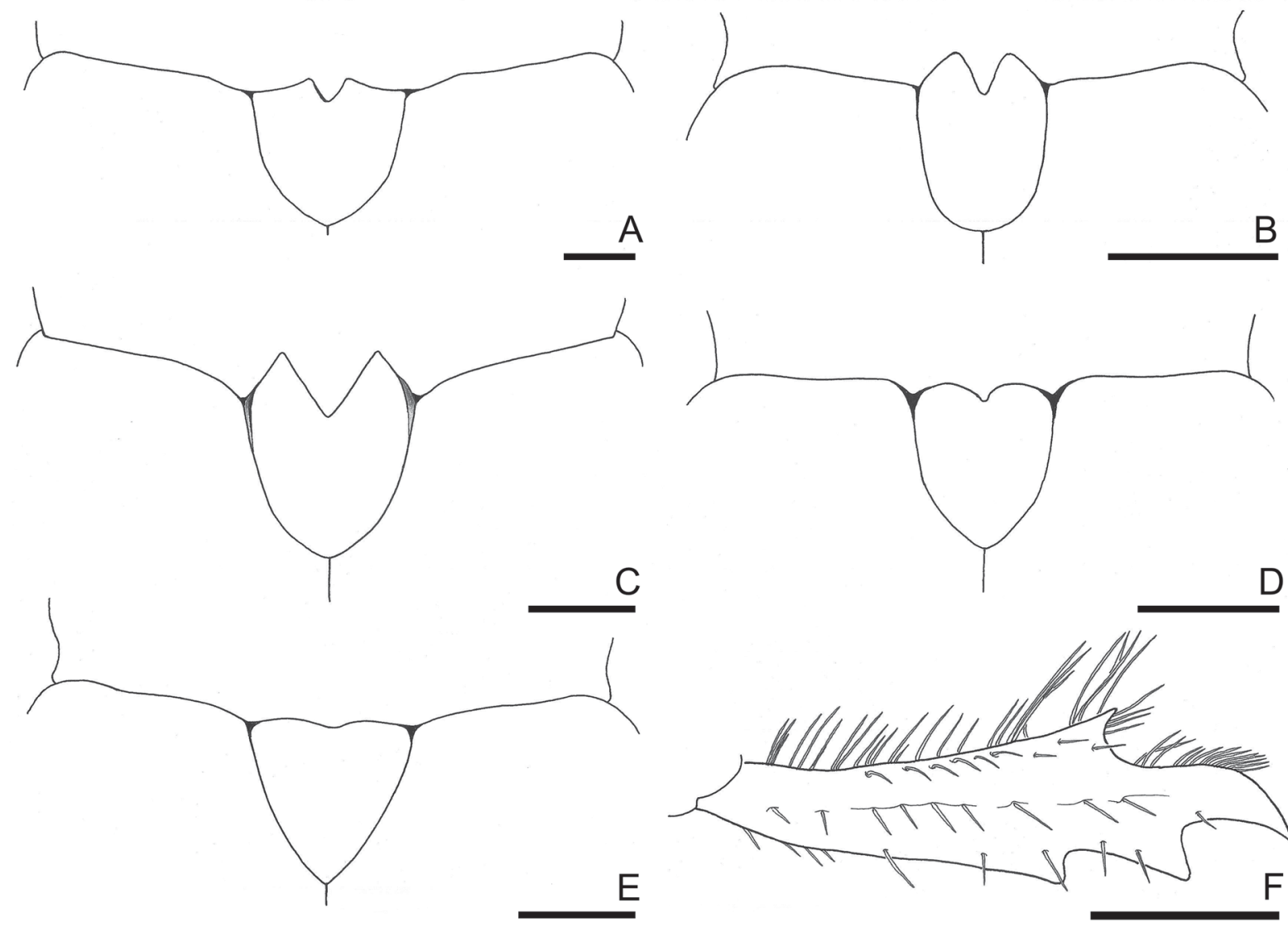

$E$

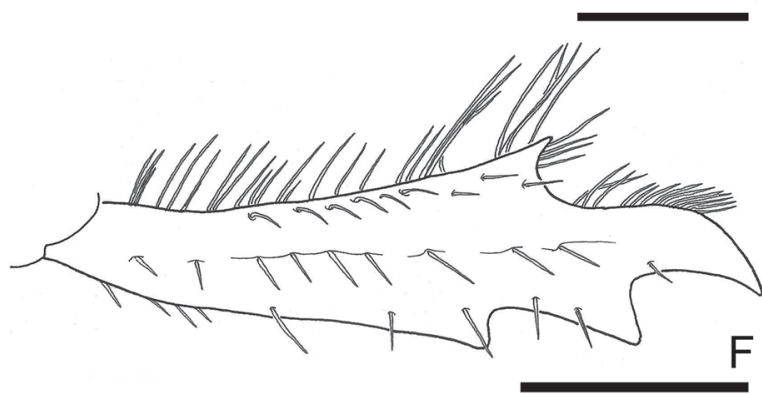

Fig. 8. A-E. Pronotum-scutellum contact. F. Protibia. A. Ancistrosoma klugii Curtis, 1835. B. Chariodema virescens Blanchard, 1842. C. Ceraspis bivulnerata (Germar, 1824). D. Faula cornuta Blanchard, 1850. E-F. Manopus biguttata Conte de Castelnau, 1840. Scale bars $=1 \mathrm{~mm}$. 
Ceraspis albida - Blanchard 1850: 92 (synonymy).

\section{Note}

Ceraspis pruinosa was described based on an undetermined number of males and females, at least one of each sex. Only one female was located and designated as lectotype.

\section{Material examined}

Lectotype (here designated, Fig. 18A-B)

BRAZIL: + , MNHN, labels: [Rio-janei. | St. Hilaire] [MUSÉUM PARIS | Rio-Janeiro | St Hilaire] [LECTOTYPE + | Ceraspis pruinosa $\mid$ LePeletier \& | Audinet-Serville, $1828 \mid$ des. J. Fuhrmann \& | F.Z. Vaz-de-Mello, 2014] [Ceraspis bivulnerata $\mid$ (Germar, 1824) o $\mid$ det. J. Fuhrmann | \& F.Z. Vaz-de-Mello, 2014].

\section{Ceratolontha Arrow, 1948}

\section{Type species}

Ceratolontha venezuelae Arrow, 1948 (designation: monotypy).

\section{Diagnosis}

Male clypeus with two long horns (Fig. 10B-C) and female clypeus deeply emarginate (Fig. 10A); maxillary palpus longer than antennomeres I-VI; pronotal anterior and posterior margins not beaded;

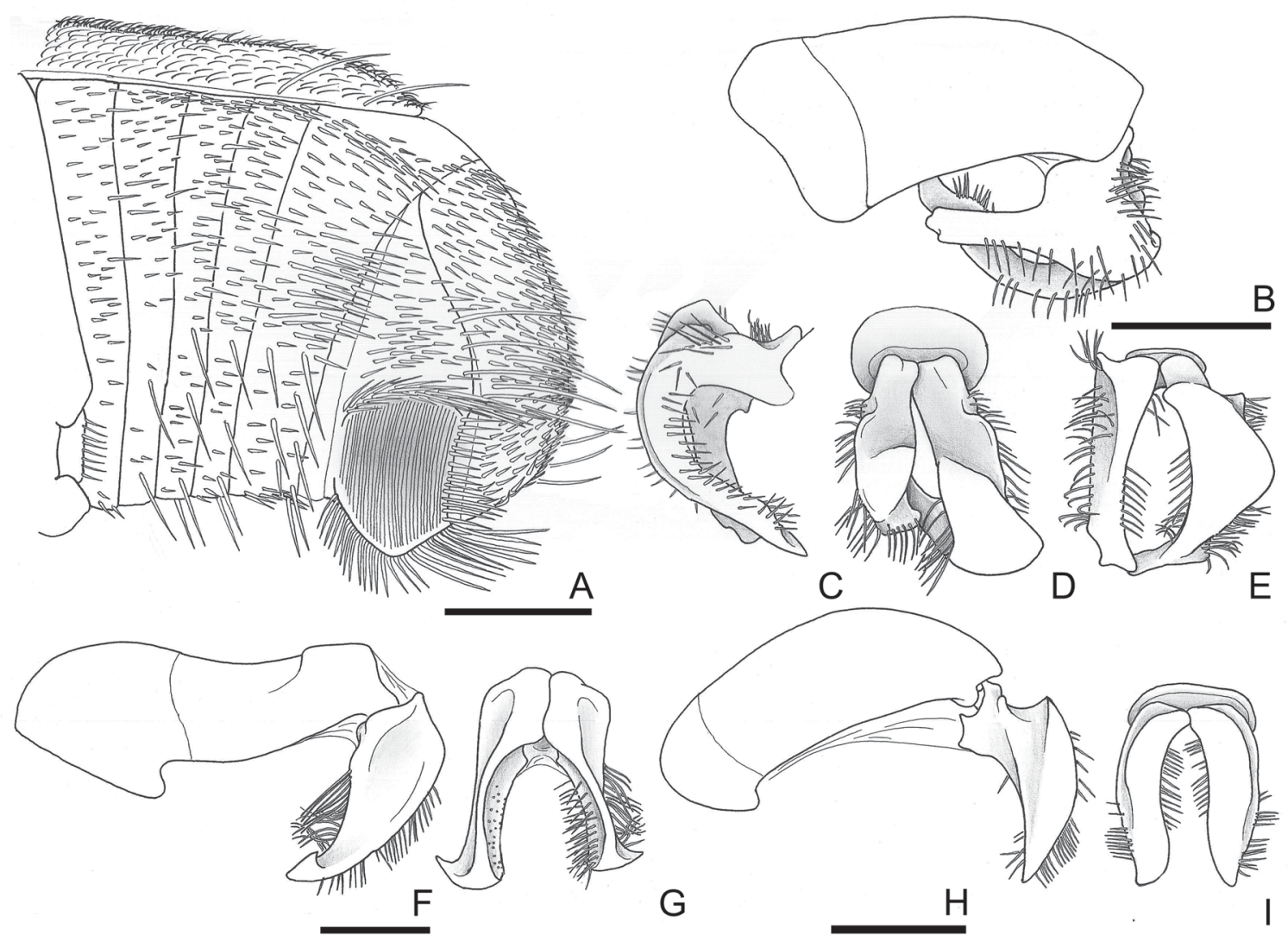

Fig. 9. A. Lateroventral detail of male abdomen. B, F, H. Aedeagus, lateral. C. Left paramere. D, G, I. Parameres, apex. E. Parameres, ventral. A-E. Chariodema virescens Blanchard, 1842. F-G. Ceraspis bivulnerata (Germar, 1824). H-I. Faula cornuta Blanchard, 1850. Scale bars $=1 \mathrm{~mm}$. 
prosternum anteriorly concave (similar to Fig. 12K); protibia with three external teeth and without spur; elytron with three raised longitudinal lines between internal margin and humerus, posterior and posterointernal margins not beaded; mesotibia with some spines; metatarsomere $\mathrm{V}$ without spine-like setae; abdomen with intersegmental membrane VII-VIII concealed.

\section{Remark}

Ceratolontha is distinguished from other Macrodactylini genera by the head ornamentation (Fig. 10B-C).

Ceratolontha venezuelae Arrow, 1948

Figs 10A-C, H-I, 18C-D

Ceratolontha venezuelae Arrow, 1948: 372.

\section{Note}

Ceratolontha venezuelae was described based on two males and one female.

\section{Material examined}

Lectotype (hereby designated, Fig. 18C-D)

VENEZUELA: §, BMNH, labels: [Type] [Venez.a] [Fry Coll. | 1095-100] [18613] [Ceratolontha | venezuelae | Type Arrow] [Ceratolontha | venezuelae Arrow | type] [LECTOTYPE | Ceratolontha | venezuelae $\mid$ Arrow, $1948|\hat{o}|$ des. des. J. Fuhrmann \& | F.Z. Vaz-de-Mello, 2014].

Paralectotypes $(\mathrm{n}=2)$

VENEZUELA: 1 o, BMNH, labels: [Venez.a | 55789.] [Ceratolontha | venezuelar | Co-type Arrow] [PARALECTOTYPE $\mid$ Ceratolontha $\mid$ venezuelae $\mid$ Arrow, 1948 $\mid$ ô $\mid$ des. J. Fuhrmann \& | F.Z. Vaz-deMello, 2014]; 1 q, BMNH, labels: [Venez.a] [Fry Coll. | 1905-100] [Ceratolontha $\mid$ venezuelae $\mid$ Cotype Arrow] [PARALECTOTYPE | Ceratolontha | venezuelae | Arrow, 1948 | $q$ | des. J. Fuhrmann \& F.Z. Vaz-de-Mello, 2014].

\section{Type species}

Chariodactylus Moser, 1919

Chariodactylus chacoensis Moser, 1919 (designation: Evans 2003: 247).

\section{Diagnosis}

Clypeus and frons gradually raised over the lateral area of head (Fig. 11A); clypeal anteroventral area strongly reduced (similar to Fig. 11H); posterior ocular area exposed (Fig. 11A), eye large; pronotum as long as wide, with anterior and posterior margins not beaded; prosternum with two anterior sulci (similar to Fig. $11 \mathrm{H}$ ); protibia with two external teeth and without spur (Fig. 11A); abdomen with intersegmental membrane VII-VIII exposed.

\section{Remarks}

The genera Chariodactylus, Macrodactylus and Manodactylus Moser, 1919 are similar to each other. Chariodactylus (Fig. 11A) is distinguished as follows (opposition to 1, Macrodactylus (Fig. 11C); 2, Manodactylus (Fig. 11B)): clypeus broad, trapezoid (1, narrow, trapezoid; 2, as Chariodactylus); eye large (1, 2, small); pronotum wider than long (1, longer than large; 2 , as Chariodactylus). Future cladistic analysis and a taxonomic review of Macrodactylus including Chariodactylus and Manodactylus are needed to confirm the possible synonymy between these genera. 

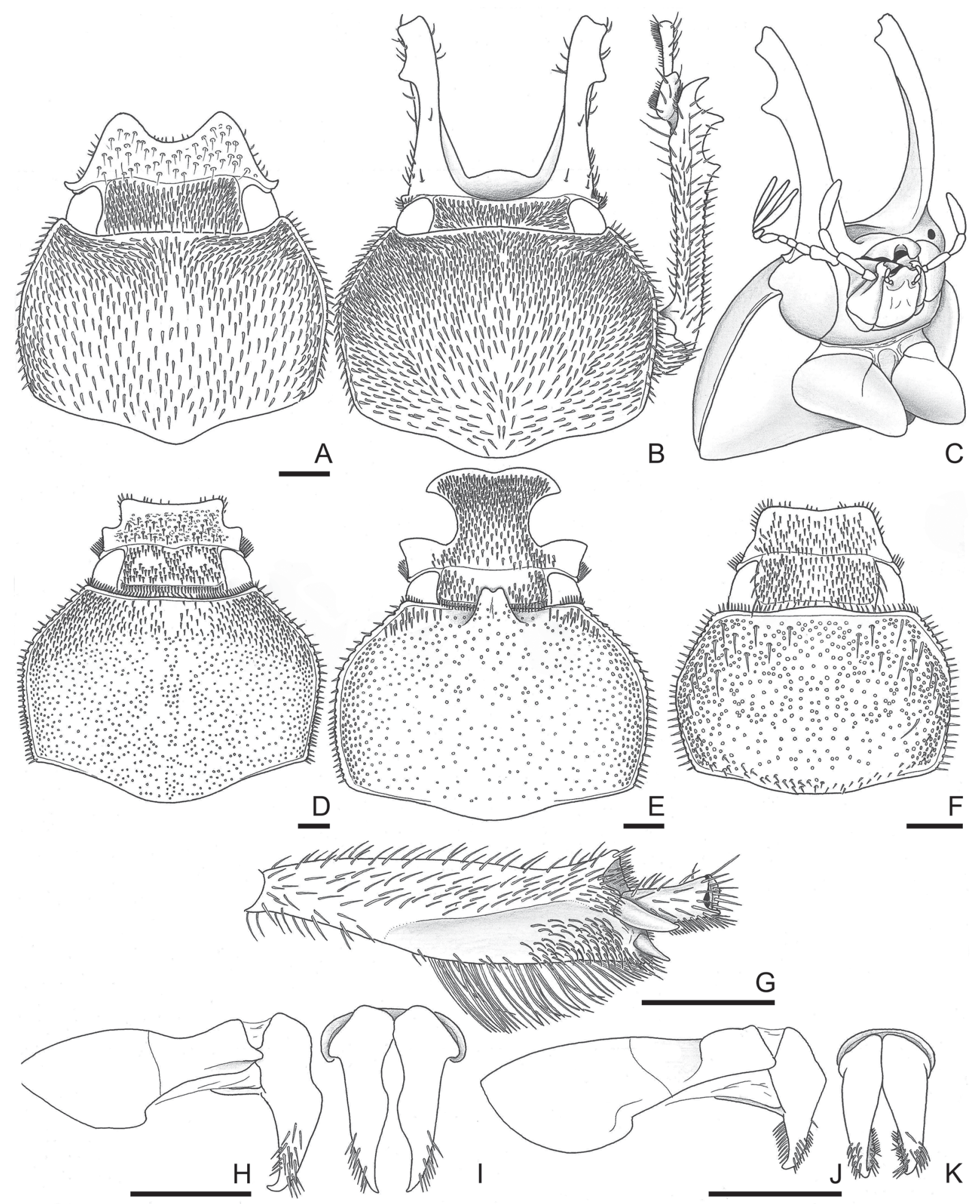

Fig. 10. A-B, D-F. Head-pronotum, dorsal. C. Head-prothorax, lateroventral. G. Metatibia, internal. A. ㅇ. B-G. O. H-K. Aedeagus (lateral, parameres apex). A-C, H-I. Ceratolontha venezuelae Arrow, 1948. D. Rhinaspis aenea Billberg, 1820. E. Rhinaspis ohausi Moser, 1921. F-G, J-K. Rhinaspis aeneofusca Moser, 1919. Scale bars $=1 \mathrm{~mm}$. 
Chariodactylus chacoensis Moser, 1919

Figs 11A, D-E, 18E-F

Chariodactylus chacoensis Moser, 1919a: 57.

\section{Note}

Chariodactylus chacoensis was described based on an undetermined number of males and females.

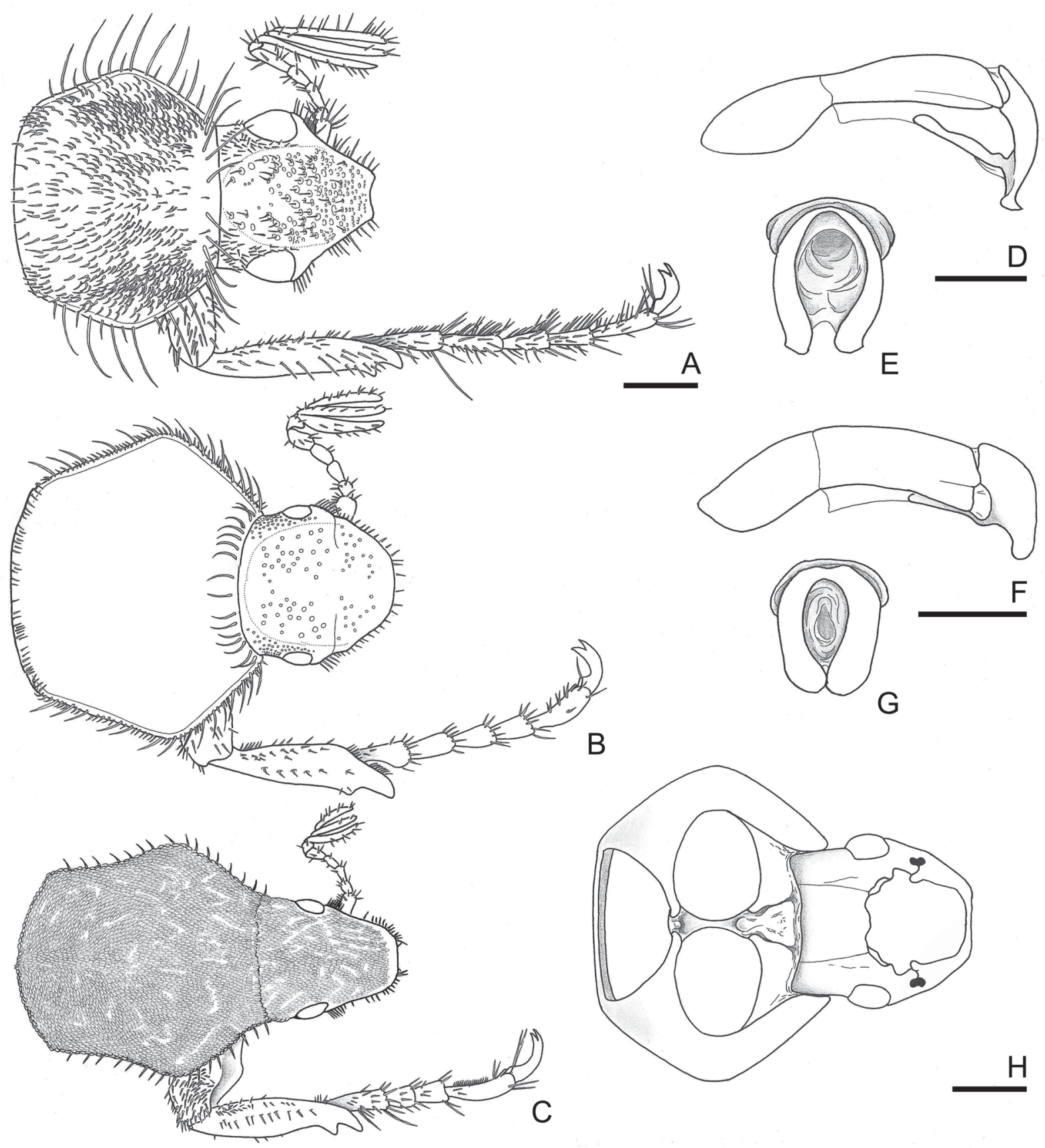

Fig. 11. $\widehat{\partial}$. A-C. Head-prothorax dorsal, tarsus rotated laterally to apex. D-G. Aedeagus (lateral, parameres apex). H. Head-prothorax, ventral. A, D-E. Chariodactylus chacoensis Moser, 1919. B, F-H. Manodactylus gaujoni Moser, 1919. C. Macrodactylus pumilio Burmeister, 1855. Scale bars = $1 \mathrm{~mm}$. 


\section{Material examined}

Lectotype (here designated, Fig. 18E-F)

BOLIVIA: $\widehat{\partial}$, ZMHU, labels: [Bolivia $\mid$ Chaco \{illegible\}] [Chariodactylus $\mid$ chacoensis $\mid$ Moser $\mid$ fide K. Katovich 02] [LECTOTYPE | Chariodactylus | chacoencis $\mid$ Moser, 1919 F.Z. Vaz-de-Mello, 2014].

\section{Paralectotype}

BOLIVIA: 1 ô, ZMHU, labels: [Bolivia | Chaco \{illegible\}] [Chariodactylus | chacoensis $\mid$ Moser | fide K. Katovich 02] [PARALECTOTYPE | Chariodactylus | chacoencis | Moser, 1919| ठै | des. J. Fuhrmann \& | F.Z. Vaz-de-Mello, 2014].

\section{Chariodema Blanchard, 1850}

Chariodema Blanchard, 1850: 117.

\section{Type species}

Philochlaenia virescens Blanchard, 1842 (designation: Evans 2003: 247).

\section{Diagnosis}

Body usually yellow or yellowish-brown, bright, and pronotum with or without green reflex (Fig. 18G); pronotal anterior margin not beaded; pronotum-scutellum contact acutely sinuous (Fig. 8B); prosternum with an anterior longitudinal carina; protibia with two external teeth and with a spur; meso- and metacoxae separate; internal area of metatarsomere V with spine-like setae; male ventrite VI with two parallel and setose carinae (Fig. 9A).

\section{Remarks}

The genera Ancistrosoma, Chariodema, Faula, Manopus, Pectinosoma and Pseudopectinosoma are similar to each other. They have a sinuous pronotum-scutellum contact and meso- and metacoxae separate. Chariodema is distinguished by protibia with two external teeth and with a spur, and male ventrite VI with two parallel and setose carinae (Fig. 9A).

Chariodema virescens (Blanchard, 1842)

Figs 8B, 9A-E, 18G-H

Philochlaenia virescens Blanchard, 1842: fig. 11.

Philochlaenia virescens - Blanchard 1845: 192.

Chariodema virescens - Blanchard 1850: 117.

\section{Note}

Philochlaenia virescens was described based on an undetermined number of specimens.

\section{Material examined}

Lectotype (here designated, Fig. 18G-H)

BOLIVIA: + , MNHN, labels: [6458 | 34] [1489] [MUSÉUM PARIS | Santa Cruz | d'Orbigny] [LECTOTYPE | q | Philochlaenia virescens | Blanchard, $1842 \mid$ des. J. Fuhrmann \& | F.Z. Vaz-de-Mello, 2014] [Chariodema virescens (Blanchard, 1842) 의 det. J. Fuhrmann \& | F.Z. Vaz-de-Mello, 2014]. 


\section{Remarks}

See remarks for Philochloenia.

\section{Clavipalpus Laporte, 1832}

Ootoma Dejean, 1833: 163, nomen nudum.

\section{Type species}

Clavipalpus dejeani Laporte, 1832 (designation: monotypy).

\section{Diagnosis}

Clypeal ventral area slightly enlarged (Fig. 6A-B); maxillary palpomere IV enlarged (Fig. 6A-B); pronotal anterior and posterior margins not beaded; prosternum anteriorly concave (similar to Fig. 12K); protibia with three external teeth and without spur; elytral striae indistinct, posterior margin not beaded, posterointernal margin beaded; metatarsomere $\mathrm{V}$ without internoproximal spine-like setae; abdomen with intersegmental membrane VII-VIII concealed.

\section{Remarks}

The present study revalidates Paulosawaya, formerly a synonym of Clavipalpus. Consequently 17 species are transferred to Paulosawaya, and Clavipalpus now comprises four species: C. dejeani, C. hirticollis Frey, 1969, C. orbignyanus Blanchard, 1850 and C. tenuis Arrow, 1903.

Clavipalpus dejeani Laporte, 1832

Figs 6A-B, E-F, 18I-J

Clavipalpus dejeani Laporte, 1832: 406.

Ootoma clavipalpe Dejean, 1837: 163, nomen nudum.

\section{Note}

Clavipalpus dejeani was described based on an undetermined number of specimens.

\section{Material examined}

Lectotype (here designated, Fig. 18I-J)

BRAZIL: $\widehat{\jmath}$, MNHN, labels: [Dejeani | Bres Castl.] [Collection Mnizech] [Ex-Musaeo | VAN LÂNSBERGE] [LECTOTYPE | Clavipalpus | dejeani | Laporte, $1832|\widehat{\jmath}|$ des. J. Fuhrmann \& | F.Z. Vaz-de-Mello, 2014].

\section{Paralectotype}

BRAZIL: 1 đે, MNHN, labels: [MUSÉUM PARIS | Castelnau] [PARALECTOTYPE | Clavipalpus | dejeani | Laporte, $1832|\widehat{\jmath}|$ des. J. Fuhrmann \& | F.Z. Vaz-de-Mello, 2014].

\section{Euryaspis Blanchard, 1850, revalidated}

Ctenotis Burmeister 1855: 20, syn. nov. Type species: Ctenotis obesa Burmeister, 1855 (designation: monotypy).

Euryaspis Lacordaire, 1856: 259 (synonymy with Plectris).

\section{Type species}

Euryaspis gaudichaudii Blanchard, 1850 (designation: monotypy). 


\section{Diagnosis}

Clypeus transverse, narrow, posterior angle partially covering the canthus (Fig. 12A), ventral area deflected vertically; pronotal anterior and posterior margins not beaded, lateral margins crenulate, posterior margin prominent (Fig. 12A), prosternum anteriorly concave (similar to Fig. 12K); protibia with three external teeth and with a spur (Fig. 12A, C); elytral striae indistinct, posterior and posterointernal margins not beaded; abdomen with intersegmental membrane VII-VIII concealed.

\section{Remarks}

The present study proposes the synonymy of Ctenotis with Euryaspis and revalidates Euryaspis, formerly a synonym of Plectris, based on the following characters (opposition to Plectris): pronotal lateral margins crenulate (margins straight), posterior margin prominent (not prominent); internal area of metatarsomere V without spine-like setae (with spine-like setae).

Euryaspis is distinguished from other Macrodactylini by having a narrow and transverse clypeus, pronotal lateral margins crenulate and posterior margin strongly prominent.

According to the new synonymy proposed here (Ctenotis synonym of Euryaspis), the genus now comprises two species: E. gaudichaudii (type species of Euryaspis) and E. obesa (Burmeister, 1855) comb. nov. (type species of Ctenotis). Euryaspis gaudichaudii is distinguished as follows (opposition to E. obesa): antennal clavae of male and females with three antennomeres (Fig. 12B) (four antennomeres, Fig. 12A); protarsomere IV about twice as long as wide (Fig. 12C) (as long as wide, Fig. 12A); protarsal claws gradually curved (Fig. 12C) (abruptly curved, Fig. 12A); male abdominal ventrites with a medial comb of long setae (setae homogeneously distributed).

Euryaspis gaudichaudii Blanchard, 1850

Figs 12B-E, 18M-N

Euryaspis gaudichaudii Blanchard, 1850: 130.

Phyllophaga (Phytalus) malleata Frey, 1964: 696.

Plectris gaudichaudii - Lacordaire 1856: 259.

Phyllophaga (Phytalus) malleata - Frey 1975: 220 (synonymy).

\section{Note}

Euryaspis gaudichaudii was described based on an undetermined number of specimens.

\section{Material examined}

Lectotype (here designated, Fig. 18M-N)

BRAZIL: $\widehat{O}$, MNHN, labels: [MUSÉUM PARIS | Bresil | Coll. Malvoidel | 1942] [TYPE] [Plectris | Euryaspis | gaudichaudi | B1. | det. G. Frey, 1965] [E. Gaudichaudii | Cat. Mus. | Brésil | M. Gaudichaud.] [LECTOTYPE $\widehat{o} \mid$ Euryaspis gaudichaudii $\mid$ Blanchard, $1850 \mid$ des. J. Fuhrmann \& | F.Z. Vaz-de-Mello, 2014].

\section{Paralectotype}

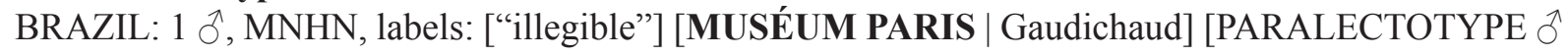
| Euryaspis gaudichaudii | Blanchard, 1850 | des. J. Fuhrmann \& | F.Z. Vaz-de-Mello, 2014]. 

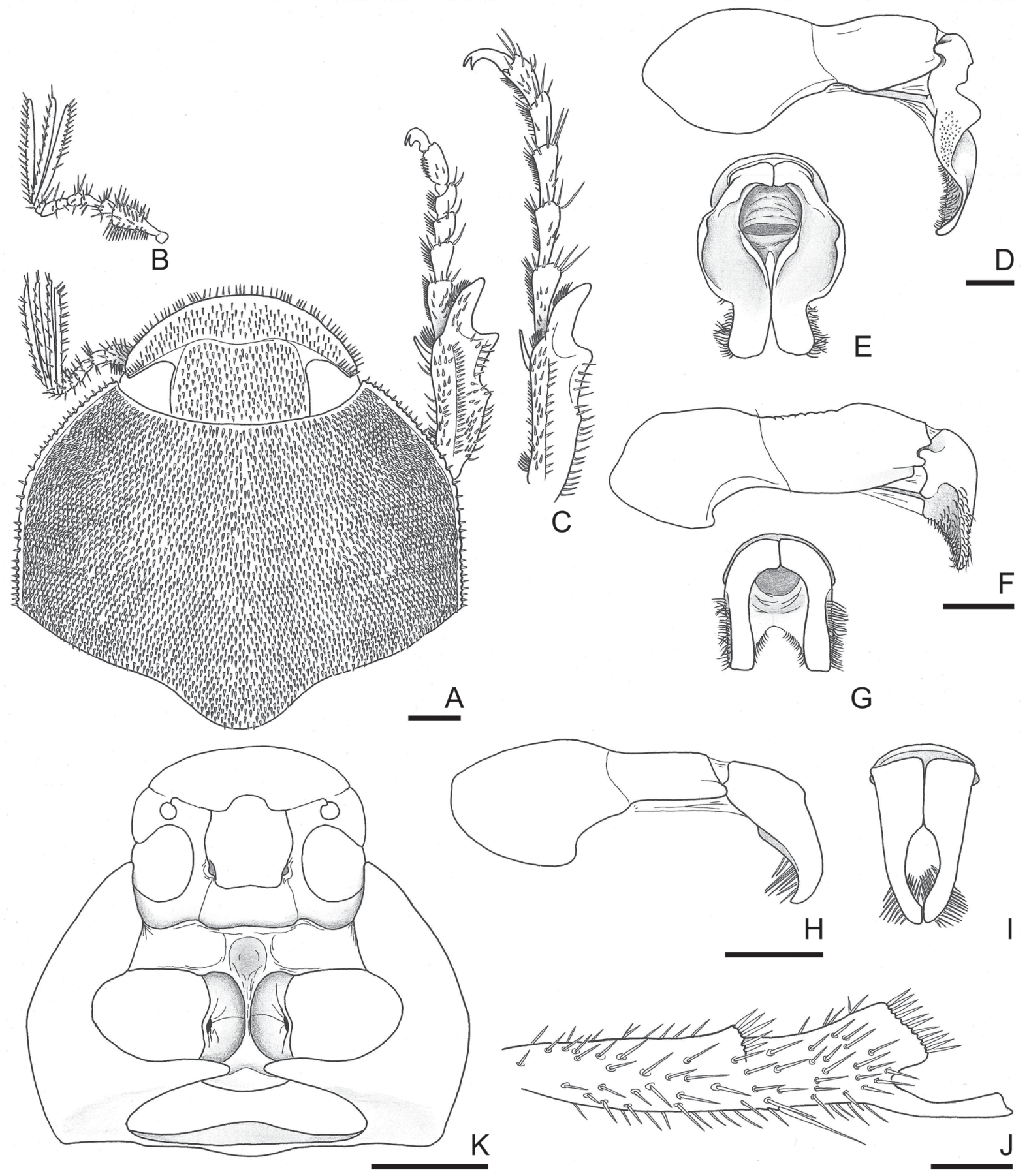

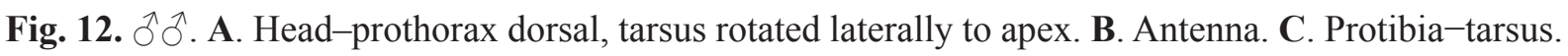
D-I. Aedeagus (lateral, parameres apex). J. Metatibia. K. Head-prothorax, ventral. A, F-G. Euryaspis obesa (Burmeister, 1855) comb. nov. B-E. Euryapsis gaudichaudi Blanchard, 1850. H-J. Philochloenia amata nom. nov. K. Philochloenia castaneipennis (Guérin-Méneville, 1831) comb. nov. Scale bars = $1 \mathrm{~mm}$. 
Euryaspis obesa (Burmeister, 1855) comb. nov.

Figs $12 \mathrm{~A}, \mathrm{~F}-\mathrm{G}, 18 \mathrm{~K}-\mathrm{L}$

Ctenotis obesa Burmeister, 1855: 21.

Phyllophaga (Phytalus) alvarengai Frey, 1964: 697.

Phyllophaga (Phytalus) alvarengai - Frey 1975: 220 (synonymy).

\section{Note}

Ctenotis obesa was described based on an undetermined number of specimens.

\section{Material examined}

Lectotype (here designated, Fig. 18K-L)

BRAZIL: $\widehat{\alpha}$, ZNSM, labels: [Ctenotis | Burm.] \{drawer label\} [obesa Br $\mid$ Rollar | Bras. inter.] \{drawer label $\}$ [LECTOTYPE | Ctenotis obesa ${ }^{\Uparrow} \mid$ burmeister, 1855 | des. J. Fuhrmann \& | F.Z. Vaz-de-Mello, 2014].

Faula Blanchard, 1850, revalidated

Faula - Lacordaire 1856: 253 (synonymy with Ancistrosoma). — Geminger \& Harold 1869: 1154 (synonymy with Ceraspis).

\section{Type species}

Faula cornuta Blanchard, 1850 (designation: Evans 2003: 236).

\section{Diagnosis}

Body usually brown or reddish-brown, opaque; pronotal anterior margin not beaded; pronotum-scutellum contact roundly sinuous (Fig. 8D); prosternum with an anterior longitudinal carina; protibia with 3-4 external teeth and without spur; meso- and metacoxae separate; metatarsomere $\mathrm{V}$ with internoproximal spine-like setae.

\section{Remarks}

The genera Ancistrosoma, Chariodema, Faula, Manopus, Pectinosoma and Pseudopectinosoma are similar to each other. They have a sinuous pronotum-scutellum contact and meso- and metacoxae separate. Faula is distinguished by the protibia with 3-4 external teeth (rarely with proximal tooth reduced) and without spur; male ventrite VI weakly prominent or flat and straight.

The present study revalidates Faula, formerly a synonym of Ceraspis, based on the following characters (opposition to Ceraspis): pronotum and elytron glabrous or sparsely setose, pronotum rarely densely setose, elytron of some species with longitudinal setose bands, at least in females (usually densely setose, sometimes setae covering the surface largely or entirely); pronotum-scutellum contact roundly sinuous (Fig. 8D) (acutely sinuous, Fig. 8C); protibia with 3-4 external teeth, some minor specimens with proximal tooth reduced (two teeth); meso- and metacoxae medially separate (contiguous).

Faula comprises 27 species ( 13 species from original combination and 14 species transferred from Ceraspis): F. bicolor (Moser, 1919) comb. nov., F. bivittata (Burmeister, 1855) comb. nov., F. brunneipennis Bates, 1887, F. centralis Sharp, 1877, F. clypealis (Frey, 1962) comb. nov., F. cornuta Blanchard, 1850, F. hispida Bates, 1887, F. immaculata (Burmeister, 1855) comb. nov., F. innotata Blanchard, 1850, F. insularis Arrow, 1903, F. jaliscoensis (Delgado \& Navarette-Heredia, 2004) comb. nov., F. klenei (Brenske, 1890) comb. nov., F. lineata Waterhouse, 1879, F. macrophylla (Moser, 1919) comb. nov., 
F. mexicana Harold, 1863, F. modesta (Burmeister, 1855) comb. nov., F. oaxacaensis (Delgado, 2001) comb. nov., F. ohausi (Moser, 1921) comb. nov., F. opacipennis Moser, 1919, F. pilatei Harold, 1863, $F$. quadrifoliata (Moser, 1919) comb. nov., F. quadrimaculata Blanchard, 1850, F. quadripustulata Blanchard, 1850, F. rubiginosa (Latreille, 1813) comb. nov., F. ruhli (Brenske, 1890) comb. nov., F. rufoscutellata (Moser, 1919) comb. nov. and F. squamulifera Moser, 1919.

Faula cornuta Blanchard, 1850

Figs 8D, 9H-I, 18O-P

Faula cornuta Blanchard, 1850: 124.

Ceraspis cornuta - Gemminger \& Harold 1869: 1154.

in Ancistrosoma (but genus combination not used) - Lacordaire 1856: 253.

Note

Faula cornuta was described based on an undetermined number of specimens.

\section{Material examined}

Lectotype (hereby designated, Fig. 180-P)

BRAZIL: đ̊, MNHN, labels: [11 | 44] [MUSÉUM PARIS | RIO-JANEIRO | DE CASTELNAU 1744] [LECTOTYPE $ð \mid$ Faula cornuta $\mid$ Blanchard, $1850 \mid$ des. J. Fuhrmann \& | F.Z. Vaz-de-Mello, 2014].

Paralectotypes $(\mathrm{n}=3)$

BRAZIL: 2 ڤે, MNHN, labels: [11 | 44] [MUSÉUM PARIS | Rio | de Castelnau] [PARALECTOTYPE $\widehat{O} \mid$ Faula cornuta | Blanchard, $1850 \mid$ des. J. Fuhrmann \& | F.Z. Vaz-de-Mello, 2014]; 1 , MNHN, labels: [11 | 44] [MUSÉUM PARIS | Rio | de Castelnau] [PARALECTOTYPE + | Faula cornuta | Blanchard, $1850 \mid$ des. J. Fuhrmann \& | F.Z. Vaz-de-Mello, 2014].

Junkia Dalla Torre, 1913, revalidated

Trichoderma Nonfried, 1894: 11 (non Stephens, 1835).

Junkia Dalla Torre, 1913: 310 (replacement name for Trichoderma Nonfried).

Junkia - Moser 1913: 293 (synonymy with Philochloenia). - Frey 1967: 79 (synonymy with Plectris).

\section{Type species}

Trichoderma ceylanica Nonfried, 1894 (monotypy).

Junkia ceylanica (Nonfried, 1894), species inquirenda

Fig. 22E

Trichoderma ceylanica Nonfried, 1894: 11.

Junkia ceylanica - Dalla Torre 1913: 310.

Philochlaenia ceylanica - Moser 1913: 293 (Junkia synonym of Philochloenia).

Plectris ceylanica - Frey 1967: 79 (Junkia synonym of Plectris).

\section{Note}

Nonfried (1894) erected Trichoderma for T. ceylanica based on an undetermined number of specimens from Kandy, Sri Lanka (“Ceylon"). Dalla Torre (1913) proposed Junkia as a replacement name for 
Trichoderma Nonfried, 1894 (non Stephens, 1848). In the same year Moser (1913) proposed the synonymy of Junkia with Philochlaenia [sic] Blanchard, 1850 and noted that the type locality "Ceylon" was probably a labelling error due to the fact that the genus occurs in South America. Frey (1967) synonymized Junkia with Plectris, and reported its occurrence in Brazil. The original collection of Nonfried is currently housed in ZMHU, but the type series of $T$. ceylanica was not found. However, two of Moser's homeotypes of the species from Paraguay were examined (ZMHU, Fig. 22E). The revalidation of Junkia proposed by the present study is based on these specimens and the original description. Further studies are needed to find or designate primary types, check the taxa systematics, and find the correct occurrence of the species.

The present study revalidates Junkia, formerly a synonym of Plectris, based on the following characters (opposition to Plectris): labrum transverse, not prominent (prominent and deeply emarginate), metatarsomere I as long as II (metatarsomere I as long as II-IV, Fig. 4A), internal area of metatarsomere $\mathrm{V}$ without spine-like setae (with spine-like setae, Fig. 4A).

Mallotarsus Blanchard, 1850

\section{Type species}

Mallotarsus spadiceus Blanchard, 1850 (designation: monotypy).

\section{Diagnosis}

Protibia with three external teeth and with a spur; mesotarsomere I with an internal hook-like tooth (Fig. 13C); metafemur with a ventral row of denticle-like setae (Fig. 13H); tarsomere IV with an internodistal small tooth (Fig. 13H); abdomen with intersegmental membrane VII-VIII exposed.

\section{Remarks}

Males of Mallotarsus have mesotarsomere I with a hook-like tooth similar to that found on Pseudoserica and part of Dicrania (e.g., D. ebenina Blanchard, 1850). Mallotarsus is distinguished as follows (opposition to 1, Dicrania; 2, Pseudoserica): pronotal posterior margin straight (1, as Mallotarsus; 2, margin with two lateromedial teeth projected between anterior elytron-scutellum contact, Fig. 13A), protibia with three external teeth and a spur (1, with two external teeth and without spur; 2, with 1-3 external teeth and with or without spur).

Mallotarsus spadiceus Blanchard, 1850

Figs 13C, F-H, 19A-B

Mallotarsus spadiceus Blanchard, 1850: 119.

\section{Note}

Mallotarsus spadiceus was described based on an undetermined number of specimens.

\section{Material examined}

Lectotype (here designated, Fig. 19A-B)

BRAZIL: Ō, MNHN, labels: [Campos | Geraes] [MUSÉUM PARIS | BRÉSIL | CAMPOS GERAES] [COTYPE] [LECTOTYPE $\hat{\jmath} \mid$ Mallotarsus spadiceus | Blanchard, 1850 | des. J. Fuhrmann \& | F.Z. Vaz-de-Mello, 2014]. 


\section{Paralectotype}

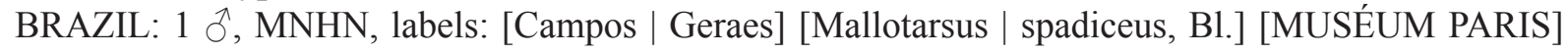
[Mallotarsus | spadiceus | Blanchard | fide K. Katovich 03] [PARALECTOTYPE | Mallotarsus spadiceus $\hat{\jmath} \mid$ Blanchard, 1850 | des. J. Fuhrmann \& | F.Z. Vaz-de-Mello, 2014].
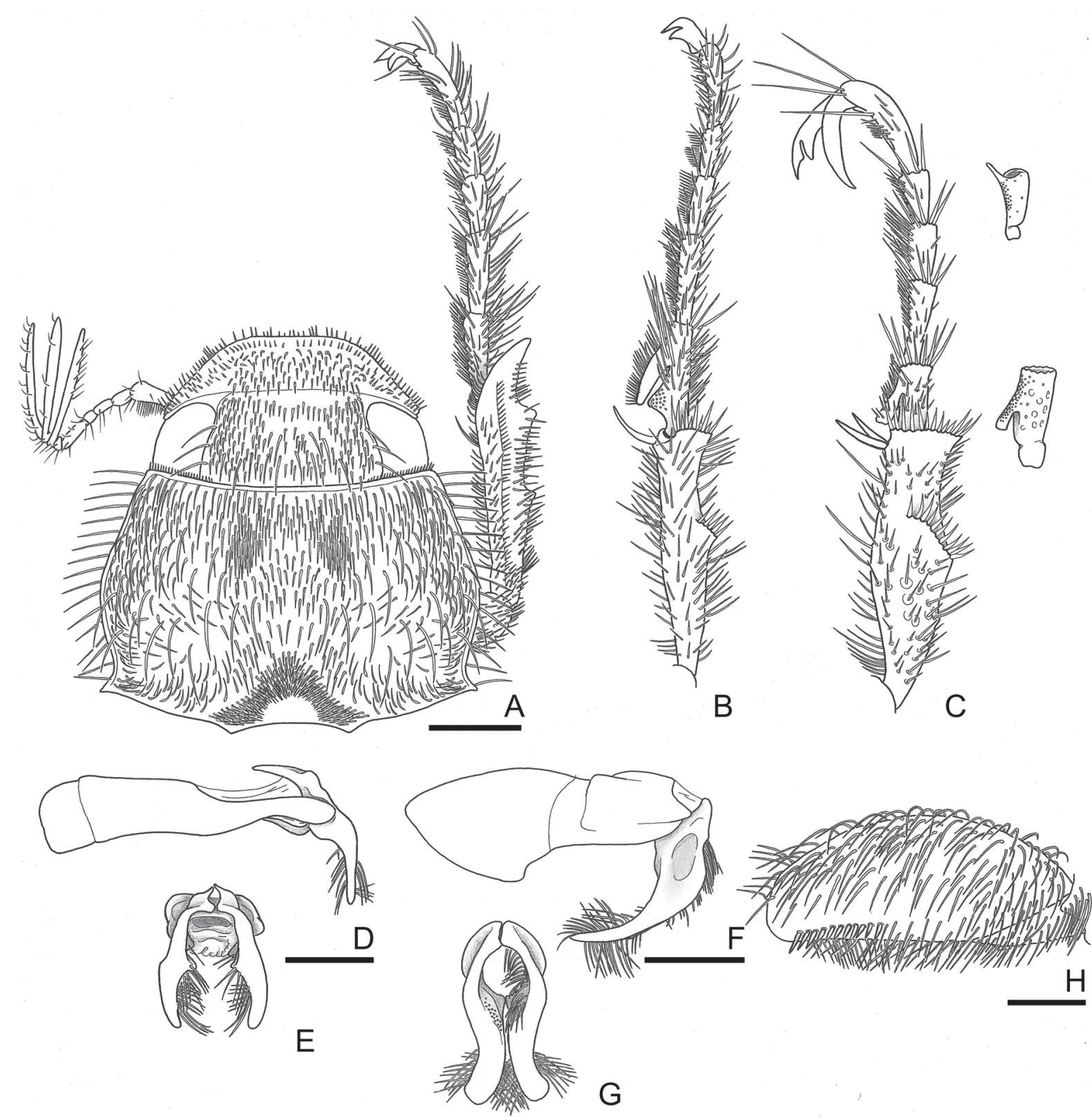

Fig. 13. $\widehat{\jmath}$. A. Head-prothorax dorsal, tarsus rotated laterally to apex. B-C. Mesotibia-tarsus (C, with details of tarsomere I and IV). D-G. Aedeagus (lateral, parameres apex). H. Metafemur. A-B, D-E. Pseudoserica marmorea Guérin-Méneville, 1831. C, F-H. Mallotarsus spadiceus Blanchard, 1850. Scale bars $=1 \mathrm{~mm}$. 
Manodactylus Moser, 1919

\section{Type species}

Manodactylus gaujoni Moser, 1919 (designation: monotypy).

\section{Diagnosis}

Clypeus and frons forming a flat subcircular abruptly raised area (Fig. 11B); clypeal anteroventral area strongly reduced (similar to Fig. 11H); posterior ocular area exposed; eye small (Fig. 11B); pronotal anterior and posterior margins not beaded and with similar width; prosternum with two anterior sulci (similar to Fig. 11H); protibia with two external teeth and without spur (Fig. 11B); abdomen with intersegmental membrane VII-VIII exposed.

\section{Remarks}

See Chariodactylus.

Manodactylus gaujoni Moser, 1919

Figs $11 \mathrm{~B}, \mathrm{~F}-\mathrm{H}, 19 \mathrm{C}-\mathrm{D}$

Manodactylus gaujoni Moser, 1919a: 44.

\section{Note}

Manodactylus gaujoni was described based on an undetermined number of males and females.

\section{Material examined}

Lectotype (here designated, Fig. 19C-D)

ECUADOR: đ̄, ZMHU, labels: [Equateur | Loja | Abbé Gaujon] [LECTOTYPE | Manodactylus | gaujoni $\mid$ Moser, $1919 \mid$ ठ | des. J. Fuhrmann \& | F.Z. Vaz-de-Mello, 2014].

Paralectotypes $(n=9)$

ECUADOR: 1 đ̆, ZMHU, labels: [Macrodactylus | gaujoni | Type Mos.] [Equateur | Loja | Abbé Gaujon] [PARALECTOTYPE | Manodactylus $\mid$ gaujoni $\mid$ Moser, $1919 \mid$ o $\mid$ des. J. Fuhrmann \& $\mid$ F.Z. Vaz-de-Mello, 2014]; 1 ô, ZMHU, labels: [Equador | Loja] [Macrodactylus | gaujoni | Type Mos.] [PARALECTOTYPE | Manodactylus $\mid$ gaujoni $\mid$ Moser, 1919 $\mid$ ô $\mid$ des. J. Fuhrmann \& $\mid$ F.Z. Vaz-deMello, 2014]; 1 đ, ZMHU, labels: [Equador | Loja] [PARALECTOTYPE | Manodactylus | gaujoni $\mid$ Moser, $1919 \mid$ ô $\mid$ des. J. Fuhrmann \& | F.Z. Vaz-de-Mello, 2014]; 1 §, ZMHU, labels: [Equateur | Loja | Abbé Gaujon] [gaujoni Mos.] [PARALECTOTYPE | Manodactylus | gaujoni | Moser, 1919

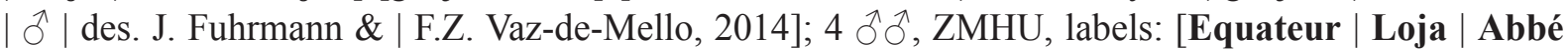
Gaujon] [PARALECTOTYPE | Manodactylus | gaujoni | Moser, 1919| ठ | des. J. Fuhrmann \& | F.Z. Vaz-de-Mello, 2014]; 1 đ, SDEI, labels: [Equateur | Loja | Abbé Gaujon] [Syntypus] [Macrodactylus | Gaujoni | Cotype Moser] \{Ohaus label\} [PARALECTOTYPE | Manodactylus | gaujoni | Moser, 1919 | $\widehat{\jmath} \mid$ des. J. Fuhrmann \& | F.Z. Vaz-de-Mello, 2014].

\section{Remarks}

The name Macrodactylus gaujoni was used by Ohaus (1909: 95) for small black specimens with long, red legs collected on "Hormillos", 3000 m, Loja Eastern Cordillera, between San Francisco River (tributary of Zamora River) and Loja municipality (Ecuador). Macrodactylus gaujoni Ohaus, 1909 was an available name, and is probably the senior synonym of Manodactylus gaujoni Moser, 1919, but the type series of the former species was not found and further studies are needed to solve this nomenclatural problem. 
Manopus Conte de Castelnau, 1840

\section{Type species}

Manopus biguttatus Conte de Castelnau, 1840 (designation: monotypy).

\section{Diagnosis}

Body brown with elytron bearing irregular dark areas, opaque; pronotal anterior margin not beaded; pronotum-scutellum contact slightly sinuous (Fig. 8E); prosternum with an anterior longitudinal carina; protibia with three external teeth and without spur, internal angle spine-like (Fig. 8F); meso- and metacoxae separate; internal area of metatarsomere $\mathrm{V}$ with spine-like setae.

\section{Remarks}

The genera Ancistrosoma, Chariodema, Faula, Manopus, Pectinosoma and Pseudopectinosoma are similar to each other. They have a sinuous pronotum-scutellum contact and separate meso- and metacoxae. Manopus is distinguished by having the pronotum-scutellum contact slightly sinuous (similar to Pectinosoma, Fig. 8E); protibia with three teeth, without spur, and with internal angle spinelike (Fig. 8F); male ventrite VI straight.

Manopus biguttatus Conte de Castelnau, 1840

Figs $8 \mathrm{E}-\mathrm{F}, 19 \mathrm{E}-\mathrm{F}$

Manopus biguttatus Conte de Castelnau, 1840: 147.

Note

Manopus biguttatus was described based on an undetermined number of specimens.

\section{Material examined}

Lectotype (here designated, Fig. 19E-F)

COLOMBIA: $\hat{\jmath}$, MNHN, labels: [MUSÉUM PARIS] [LECTOTYPE | Manopus biguttatus | Laporte, 1840 | des. J. Fuhrmann \& | F.Z. Vaz-de-Mello, 2014].

Paralectotypes $(\mathrm{n}=2)$

COLOMBIA: $1 \hat{\jmath}$, MNHN, labels: [MUSÉUM PARIS] [PARALECTOTYPE | Manopus biguttatus | Laporte, 1840 | des. J. Fuhrmann \& |F.Z. Vaz-de-Mello, 2014]; 1 §̊, MNHN, labels: [11 | 44] [MUSÉUM PARIS | "illegible" | de Castelnau] [COTYPE] [PARALECTOTYPE | Manopus biguttatus | Laporte, 1840 | des. J. Fuhrmann \& | F.Z. Vaz-de-Mello, 2014].

Oedichira Burmeister, 1855

\section{Type species}

Oedichira pachydactyla Burmeister, 1855 (designation: Evans 2003: 303).

\section{Diagnosis}

Antennae with four lamellae (Fig. 4I-J); clypeal ventral area large and vertically deflected; labrum deeply emarginate; pronotal anterior and posterior margin not beaded; prosternum with an anterior longitudinal carina; elytral striae indistinct, posterior and posterointernal margins not beaded; metatarsomere I as long as II-IV; metatarsomere II wider than long; metatarsomere V with internoproximal spine-like setae (Fig. 4C-D). 


\section{Remarks}

See Anomonyx (above).

Oedichira pachydactyla Burmeister, 1855

Figs 4C-D, G-J, 19G-H

Oedichira pachydactyla Burmeister, 1855: 81.

\section{Note}

Oedichira pachydactyla was described based on an undetermined number of specimens.

\section{Material examined}

Lectotype (here designated, Fig. 19G-H)

BRAZIL: $\hat{\jmath}$, ZNSM, labels: [Oedichira | Burm.] \{drawer label\} [pachydactyla | Bras. Br.] \{drawer label\} [LECTOTYPE | Oedichira pachydactyla $\mid$ Burmeister, $1855|\hat{~} \hat{\sigma}|$ des. J. Fuhrmann \& | F.Z. Vazde-Mello, 2014].

Paralectotypes $(n=2)$

BRAZIL: 1 + ZNSM, labels: [Oedichira|Burm.] \{drawer label\} [pachydactyla| Bras. Br.] \{drawer label\} [Nov.|Frib.] [Oedichira $\mid$ pachydactylla $\mid$ Burmeister $\mid$ fide K. Katovich 2002] [PARALECTOTYPE | Oedichira pachydactyla | Burmeister, $1855|q|$ des. J. Fuhrmann \& | F.Z. Vaz-de-Mello, 2014]; 1 + , ZNSM, labels: [Oedichira | Burm.] \{drawer label\} [pachydactyla | Bras. Br.] \{drawer label\} [PARALECTOTYPE | Oedichira pachydactyla | Burmeister, $1855 \mid$ o $\mid$ des. J. Fuhrmann \& | F.Z. Vazde-Mello, 2014].

Paulosawaya Martínez \& d'Andretta, 1956, revalidated

Amphicrania Burmeister, 1855: 26 (non Amphicania Dejean, 1833, a synonym of Liogenys, Diplotaxini), syn. nov. Type species: Amphicrania ursina Burmeister, 1855 (designation: Evans 2003: 249); junior synonymy of Paulosawaya ursina (Blanchard, 1850) comb. nov. (original combination: Clavipalpus ursinus Blanchard, 1850).

Pseudoleuretra Martínez \& d'Andretta, 1956: 351, syn. nov. Type species: Pseudoleuretra bokermanni Martínez \& d'Andretta, 1956 (original designation).

Amphicrania - Gemminger \& Harold 1869: 1143 (synonymy with Clavipalpus).

Paulosawaya - Smith 2008: 22 (synonymy with Clavipalpus).

\section{Type species}

Paulosawaya ornatissima Martínez \& d'Andretta, 1956 (original designation).

\section{Diagnosis}

Clypeal ventral area strongly widened; maxillary palpomere IV elongate (Fig. 6D); pronotal anterior and posterior margins not beaded; prosternum anteriorly concave (similar to Fig. 12K); protibia with 2-3 external teeth and without spur; elytral striae indistinct, posterior and posterointernal margins not beaded; meso- and metatibiae each with a transverse carina; metatarsomere V without spine-like setae; abdomen with intersegmental membrane VII-VIII concealed. 


\section{Remarks}

Katovich (2008) redescribed Clavipalpus based on syntypes of C. ursinus Blanchard, 1850 and the holotype of $C$. aequatorialis Moser, 1918. Thereafter, Smith (2008) proposed the synonymy of Paulosawaya with Clavipalpus based on the study of P. ornatissima (type species of Paulosawaya) and Clavipalpus spp. Clavipalpus ursinus, C. aequatorialis and P. ornatissima evidently belong to the same genus as stated by Smith (2008), but the problem is that the type material of the type species of the genus Clavipalpus (C. dejeani) was not examined by these studies (Katovich 2008; Smith 2008).

The present study designates a lectotype for Clavipalpus dejeani and Amphicrania ursinus, revalidates Paulosawaya (type species: P. ornatissima, Fig. 22A-B), and proposes the synonymy of Amphicrania Burmeister and Pseudoleuretra (type species: P. bokermanni, Fig. 22C-D) with Paulosawaya. Amphicrania Burmeister is a senior synonym of Paulosawaya, but it is a junior homonym of Amphicrania Dejean (a synonym of Liogenys according to Erichson 1848) and may not be used as a valid name (ICZN 1999: Articles 23.3.5. and 52.2).

Paulosawaya now comprises 17 species and one subspecies: P. aequatorialis (Moser, 1918) comb. nov., P. antisanae (Bates, 1891) comb. nov., P. basalis (Moser, 1918) comb. nov., P. blanchardi (Kirsch, 1885) comb. nov., P. bokermanni (Martínez \& d'Andretta, 1956) comb. nov., P. castanea (Moser, 1924) comb. nov., $P$. cochleata (Burmeister, 1855) comb. nov., $P$. hirsuta (Kirsch, 1885) comb. nov., P. ornatissima Martínez \& d'Andretta, 1956, P. peruana (Moser, 1918) comb. nov., P. rimbachi (Moser, 1924) comb. nov., P. sinuatus (Kirsch, 1885) comb. nov., P. spadicea (Burmeister, 1855) comb. nov., P. ursina (Blanchard, 1850) comb. nov., $P$. variolosa (Burmeister, 1855) comb. nov., $P$. vestita (Moser, 1924) comb. nov., $P$. whymperi chimborazonus (Bates, 1891) comb. nov. and $P$. whymperi whymperi (Bates, 1891) comb. nov.

The present study revalidates Paulosawaya, formerly a synonym of Clavipalpus, based on the following characters (opposition to Clavipalpus): clypeus broad and semicircular or truncated (narrow and trapezoid); clypeal posterior angle not extended over the canthus (partially covering the canthus); maxillary palpus long (Fig. 6D) (short, Fig. 6B), palpomere IV distinctly enlongate (Fig. 6D) (enlarged, Fig. 6B); meso- and metatibiae each with a raised transverse carina (carina distinct by large punctation). Paulosawaya usually includes large specimens (over $12 \mathrm{~mm}$ ), that are reddish-brown, with long setae and occuring in northern Brazil, Guyana, Peru, Ecuador and Colombia, and Clavipalpus includes medium-sized specimens (about $9 \mathrm{~mm}$ ), yellowish-brown, with short setae and occuring in Brazil.

Paulosawaya ursina (Blanchard, 1850) comb. nov.

Figs $6 \mathrm{C}-\mathrm{D}, 19 \mathrm{I}-\mathrm{J}$

Clavipalpus ursinus Blanchard, 1850: 120.

Amphicrania ursina Burmeister, 1855: 27 (different species, with the same epithet, later synonymized).

Amphicrania ursina - Gemminger \& Harold 1869: 1143 (synonymy).

\section{Note}

Amphicrania ursina Burmeister was described based on an undetermined number of specimens.

\section{Material examined}

Lectotype (here designated, Fig. 19I-J)

COLOMBIA: $\widehat{\partial}$, ZNSM, labels: [Amphicra- $\mid$ nia* $\mid$ Dej. ex prt.] \{drawer label\} [ursina $\mid$ Philochl. urs. | Dej. Bq. | Columb. 144.] \{drawer label\} [LECTOTYPE | Amphicrania ursina | Burmeister, 1855 | $\widehat{~ \mid ~}$ des. J. Fuhrmann \& | F.Z. Vaz-de-Mello, 2014]. 
Paralectotypes $(\mathrm{n}=2)$

COLOMBIA: 1 ○े, ZNSM, labels: [Amphicra- $\mid$ nia* $\mid$ Dej. ex prt.] \{drawer label\} [ursina $\mid$ Philochl. urs. | Dej. Bq. | Columb. 144.] \{drawer label\} [HALLA | ARRIPHICRANIA | URSINA | BURMEISTER] [Clavipalpus | ursinus | (Burmeister) | Det. K. Katovich 2002] [PARALECTOTYPE | Amphicrania ursina $\mid$ Burmeister, $1855|\widehat{\jmath}|$ des. J. Fuhrmann \& | F.Z. Vaz-de-Mello, 2014]; 1 + , ZNSM, labels: [Amphicra- | nia* | Dej. ex prt.] \{drawer label\} [ursina | Philochl. urs. | Dej. Bq. | Columb. 144.] \{drawer label\} [PARALECTOTYPE |Amphicrania ursina | Burmeister, $1855 \mid$ o $\mid$ des. J. Fuhrmann \& | F.Z. Vazde-Mello, 2014].

Pectinosoma Arrow, 1913

\section{Type species}

Pectinosoma elongata Arrow, 1913 (designation: monotypy).

\section{Diagnosis}

Pronotum-scutellum contact slightly sinuous (similar to Fig. 8E); male mesotarsomeres I-IV wider than long and with external projection; internal area of metatarsomere $\mathrm{V}$ with spine-like setae; male ventrite $\mathrm{V}$ with medial lobe bearing truncate setae in transverse palisade (Fig. 14D-E); female pygidium with sinuous apex (Fig. 14F-G).

\section{Remarks}

The genera Ancistrosoma, Chariodema, Faula, Manopus, Pectinosoma and Pseudopectinosoma are similar to each other. They have a sinuous pronotum-scutellum contact and separate meso- and metacoxae. In Ancistrosoma and Pectinosoma the prosternum has a prominent posterior process (simple in Ancistrosoma (Fig. 14A); bifid in Pectinosoma). Other distinctive characters of Pectinosoma are (opposition to 1, Ancistrosoma; 2, Pseudopectinosoma): pronotum-scutellum contact slightly sinuous (similar to Fig. 8E) (1,2, strongly sinuous, Fig. 8A); male ventrite $\mathrm{V}$ with medial lobe bearing truncate setae in transverse palisade (Fig. 14D-E) (1, ventrite I with an acute process (Fig. 14A); 2, ventrite VI with a raised area); female pygidial anterior margin straight and posterior margin deeply sinuous (Fig. 14F-G) (1, anterior margin sinuous and posterior margin slightly sinuous (Fig. 14B-C); 2, anterior margin straight and posterior margin slightly sinuous). The notes about Pseudopectinosoma are based on its original description (Katovich 2011).

Pectinosoma elongata Arrow, 1913

Figs 14D-I, 19K-L

Pectinosoma elongata Arrow, 1913: 430.

\section{Note}

Pectinosoma elongata was described based on an undetermined number of males.

\section{Material examined}

Lectotype (here designated, Fig. 19K-L)

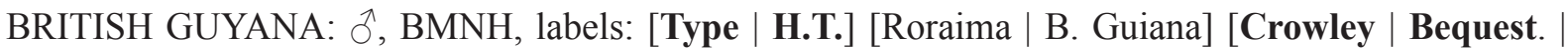
1901-78] [Pectinosoma | elongatum | Type Arrow] [LECTOTYPE | Pectinosoma | elongata | Arrow, $1913|\hat{o}|$ des. J. Fuhrmann | F.Z. Vaz-de-Mello, 2014]. 

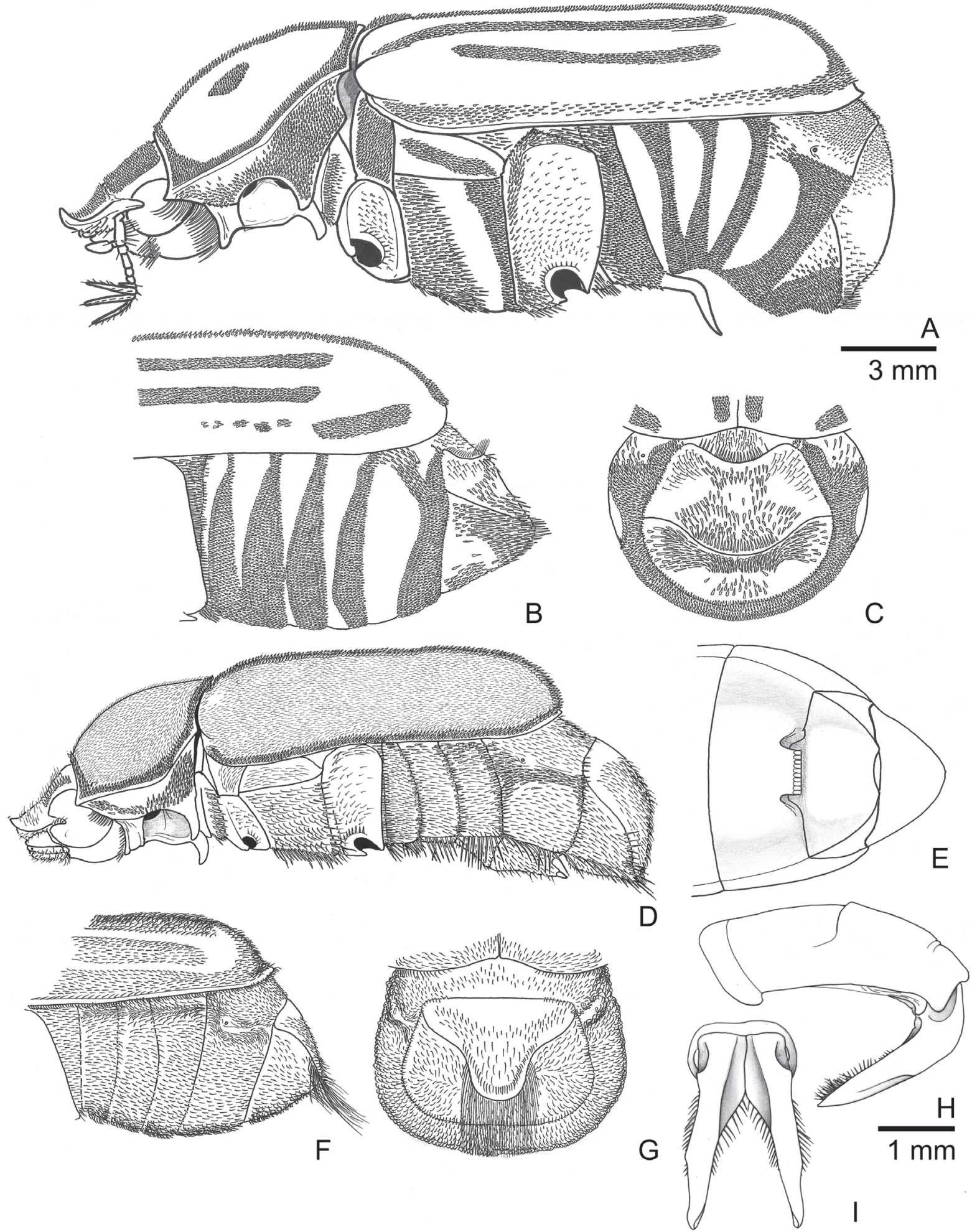

Fig. 14. A, D. Male habitus, lateral (without some appendages). B-C, F-G. Female abdomen detail (lateral, posterior). E. Male abdomen detail, ventral. H-I. Aedeagus (lateral, parameres apex). A-C. Ancistrosoma klugii Curtis, 1835. D-I. Pectinosoma elongata Arrow, 1913. 
Paralectotypes $(\mathrm{n}=3)$

BRITISH GUYANA: 1 ô, BMNH, labels: [Roraima | B. Guiana] [Crowley | Bequest. | 1901-78] [PARALECTOTYPE | Pectinosoma | elongata | Arrow, 1913 | ठ | des. J. Fuhrmann | F.Z. Vaz-de-Mello, 2014]; 2 $\widehat{\jmath}$, BMNH, labels: [Roraima | B. Guiana] [Crowley | Bequest. | 1901-78] [Pectinosoma | elongata $\mid$ Arrow | fide K. Katovich 02] [PARALECTOTYPE | Pectinosoma | elongata | Arrow, 1913 | $\widehat{O} \mid$ des. J. Fuhrmann | F.Z. Vaz-de-Mello, 2014].

Philochloenia Dejean, 1833

Philochloenia Dejean, 1833: 163.

Pachycerus Guérin-Méneville, 1831: fig. 3 (non Schoenherr 1823). Type species: Pachycerus castaneipennis Guérin-Méneville, 1831 (designation: monotypy).

Anoplosiagum Blanchard, 1850: 119. Type species: Melolontha rufipennis Fabricius, 1801 (designation: Lacordaire 1856: 264).

Aulanota Moser, 1924: 157, syn. nov. Type species: Aulanota sulcipennis Moser, 1924 (designation: monotypy), (now Philochloenia armata nom. nov.).

Hadrocerus Guérin-Méneville, 1838: 83 (replacement name for Pachycerus Guérin-Méneville), syn. nov. Anomaloptera Burmeister, 1855: 22, nomen nudum.

Philochloenia - Frey 1967: 3 (synonymy with Plectris). - Smith \& Evans 2005: 40 (synonymy with Dichelonyx). - Bousquet \& Bouchard 2013: 39 (revalidated).

Anoplosiagum - Bousquet \& Bouchard 2013: 39 (synonymy with Philochloenia).

\section{Type species}

Melolontha filitarsis Germar, 1824 (designation: Chevrolat: 735); junior synonym of Melolontha rufipennis Fabricius, 1801 (type species of Anoplosiagum).

\section{Diagnosis}

Clypeal ventral area large (Fig. 12K); pronotal anterior and posterior margins not beaded; prosternum anteriorly concave (Fig. 12K); elytral striae shallowly concave and punctate, five elytral striae present between internal margin and humerus, anterior and posterior margins not beaded and internal and posterointernal margins beaded; metatarsomere $\mathrm{V}$ with internoproximal spine-like setae.

\section{Remarks}

The genus has the pronotum with irregularly distributed large punctures. Some important interspecific variations are as follows: eye small (interocular area about four times wider than dorsal eye width, e.g., Philochloenia castaneipennis comb. nov., P. rufipennis comb. nov.) or large (interocular area about twice width of dorsal eye width, e.g., P. sulcatula (Blanchard, 1850) comb. nov.); antennae with eight (e.g., $P$. armata nom. nov.) or nine (e.g., $P$. castaneipennis comb. nov.) antennomeres; protibia with two (e.g., P. castaneipennis comb. nov.) or three (e.g., P. armata nom. nov.; P. rufipennis comb. nov.) external teeth; protibia with (e.g., P. rufipennis comb. nov.) or without (e.g., P. castaneipennis comb. nov., $P$. armata nom. nov.) a spur.

Philochloenia Dejean, 1833 was an available name with remarkable historical problems of misspelling and misapplication (see Smith \& Evans 2005: 41). Bousquet \& Bouchard (2013) discussed two type species designations of Philochloenia: Melolontha filitarsis Germar, 1824 (a synonym of Melolontha rufipennis, the type species of Anoplosiagum) according to Chevrolat (1847), which made Philochloenia the senior synonym of Anoplosiagum (current valid classification); and Melolontha elongata Fabricius, 1792 (now Dichelonyx elongatula Schoenherr, 1817) according to Smith \& Evans (2005) which made Philochloenia a junior synonym of Dichelonyx Harris, 1827 (Dichelonychini). 
Another type species designation for Philochloenia was proposed by Desmarest (1860: 69), but the type species was fixed on an unavailable name: Philochloenia virescens Dejean, 1833, nomen nudum. Blanchard $(1842,1845,1850)$ used this name to describe two species: Philochlaenia [sic] virescens Blanchard 1850: 122, a homonym of Philochlaenia [sic] virescens Blanchard, 1842 (type species of Chariodema; see Blanchard 1850: 117) and a synonym of Plectris ahena (Burmeister, 1855) (synonymy by Frey 1967: 114; correct name precedence by Evans 2003: 306).

The present study proposes the synonymy of Aulanota and Hadrocerus with Philochloenia.

Philochloenia armata Fuhrmann \& Vaz-de-Mello, 2017 nom. nov. Figs 12J, 19M-N

Aulanota sulcipennis Moser, 1924: 157.

Note

Aulanota sulcipennis was described based on an undetermined number of males.

\section{Etymology}

The specific epithet refers to Latin 'armata': armed, and alludes to the metatibial ornamentation.

\section{Material examined}

Lectotype (here designated, Fig. 19M-N)

BRAZIL: $\widehat{O}$, ZMHU, labels: [Petropolis | 23.II.99] [Aulanota | sulcipennis | Type Mos] [sulcipennis Mos] [LECTOTYPE $\mid$ Aulanota $\mid$ sulcipennis $\mid$ Moser, $1924|\widehat{~}|$ des. J. Fuhrmann \& | F.Z. Vaz-de-Mello, 2014].

\section{Remarks}

The new replacement name Philochloenia armata nom. nov. is hereby erected for Aulanota sulcipennis to avoid the secondary homonymy with Philochloenia sulcipennis (Moser, 1918) comb. nov. (described as Anoplosiagum sulcipenne). See Discussion "Male morphology and nomenclature" (below) for more information about the new synonymy.

Philochloenia rufipennis (Fabricius, 1801) comb. nov.

Fig. 19O-P

Melolontha rufipennis Fabricius, 1801: 167

Melolontha filitarsis Germar, 1824: 125.

Anoplosiagum rufipenne - Guérin-Méneville 1838: 83.

Melolontha filitarsis - Blanchard 1850: 119 (synonymy).

\section{Note}

Melolontha rufipennis was described based on an undetermined number of specimens.

\section{Material examined}

Lectotype (here designated, Fig. 19O-P)

BRAZIL: Õ, MNHN, labels: [rufipennis | Brasil “illegible"] [MUSÉUM PARIS | Collection | "illegible”] [Anoplosiagum | !sucatulum | Bl.] [LECTOTYPE $\widehat{\jmath} \mid$ Melolontha rufipennis | Fabricius, $1801 \mid$ des. 
J. Fuhrmann \& | F.Z. Vaz-de-Mello, 2014] [Philochloenia $\mid$ rufipennis đ̊ $\mid$ (Fabricius, 1801) | det. J. Fuhrmann \& | F.Z. Vaz-de-Mello, 2014].

\section{Remarks}

Most of Fabricius' types are currently housed at Staten Naturrhistoriske Museum (Copenhagen, Denmark), but the type series of Melolontha rufipennis was part of the Lous Bosc collection, now housed in NMHN (Cambefort 2006). Fabricius' original labels were identified using Horn (1990a, b).

Philochloenia castaneipennis (Guérin-Méneville, 1831) comb. nov.

Figs $12 \mathrm{~K}, 20 \mathrm{~A}-\mathrm{B}$

Pachycerus castaneipennis Guérin-Méneville, 1831: fig. 3.

Hadrocerus castaneipennis - Guérin-Méneville 1838: 83.

\section{Note}

Pachycerus castaneipennis was described based on an undetermined number of specimens.

\section{Material examined}

Lectotype (here designated, Fig. 20A-B)

BRAZIL: Ō, MNHN, labels: [St. Catherine | Brésil | Durville] [MUSÉUM PARIS] [TYPE] [Pachycerus | castaneipennis | Guerin "illegible" | Duperris pl.3 f.8] [P. castaneipennis | "illegible" | St. Catherine | Durville] [LECTOTYPE đ̊ | Pachycerus castaneipennis | Guérin-Méneville, 1831 | des. J. Fuhrmann \& | F.Z. Vaz-de-Mello, 2014] [Philochloenia castaneipennis | (Guérin-Méneville, 1831) $\widehat{o} \mid$ det. J. Fuhrmann \& | F.Z. Vaz-de-Mello, 2014].

Plectris LePeletier de Saint-Fargeau \& Audinet-Serville, 1828

Rhizonemus Dejean, 1836b: 180, nomen nudum.

Anomalochilus Blanchard, 1850: 123, syn. nov. Type species: Anomalochilus singularis Blanchard, 1850 (designation: monotypy).

Demodema Blanchard, 1850: 121. Type species: Demodema fallax Blanchard, 1850 (designation: Lacordaire 1856: 258).

Demodema - Katovich 2008: 6 (synonymy).

\section{Type species}

Plectris tomentosa LePeletier de Saint-Fargeau \& Audinet-Serville, 1828 (designation: monotypy).

\section{Diagnosis}

Labrum deeply emarginate; pronotal anterior and posterior margins not beaded; prosternum with an anterior longitudinal carina; elytral striae indistinct, elytral posterior and posterointernal margins not beaded; metatarsomere I as long as II-IV; metatarsomere VI longer than wide; metatarsomere V with internoproximal spine-like setae (Fig. 4A).

\section{Remarks}

See Anomonyx (above). Plectris is the biggest and most problematic genus of Macrodactylini. The diagnosis provided by the present study or by Katovich (2008) do not include all species, and the review of Frey (1967) did not define the genus clearly. The species richness, taxonomic problems, and 
the economic importance (e.g., Roberts 1968; Brill \& Abney 2013) of Plectris show the urgency for systematic works on the genus.

The present study proposes the synonymy of Anomalochilus with Plectris, and two species previously placed in Anomalochilus are transferred to Plectris: P. pilosella (Blanchard, 1850) comb. nov. and P. singularis comb. nov.

Plectris fallax (Blanchard, 1850)

Fig. 20E-F

Demodema fallax Blanchard, 1850: 121.

Plectris fallax - Katovich 2008: 6 (Demodema synonym of Plectris).

\section{Note}

Demodema fallax was described based on an undetermined number of specimens.

\section{Material examined}

Lectotype (here designated, Fig. 20E-F)

BRAZIL: $\widehat{\lambda}$, MNHN, labels: [14 | "illegible"] [MUSÉUM PARIS | BRÉSIL | MINAS-GERAES | DE CASTELNAU 1847] [COTYPE] [Demodema fallax B1.] [LECTOTYPE $\hat{\sigma}$ | Demodema fallax | Blanchard, $1850 \mid$ des. J. Fuhrmann \& | F.Z. Vaz-de-Mello, 2014] [Plectris fallax | (Blanchard, 1850)| det. J. Fuhrmann \& | F.Z. Vaz-de-Mello, 2014].

\section{Paralectotype}

BRAZIL: 1 ภे, MNHN, labels: [14 | "illegible"] [MUSÉUM PARIS | BRÉSIL | DE CASTELNAU] [PARALECTOTYPE $\widehat{\jmath} \mid$ Demodema fallax | Blanchard, $1850 \mid$ des. J. Fuhrmann \& | F.Z. Vaz-de-Mello, 2014] [Plectris fallax $\mid$ (Blanchard, 1850) $|\hat{O}|$ det. J. Fuhrmann \& | F.Z. Vaz-de-Mello, 2014].

\section{Plectris singularis (Blanchard, 1850) comb. nov.}

Fig. 20C-D

Anomalochilus singularis Blanchard, 1850: 123.

\section{Note}

Anomalochilus singularis was described based on an undetermined number of specimens.

\section{Material examined}

Lectotype (Fig. 20C-D)

BRAZIL: Ō, MNHN, labels: [5215 | 34.] [MUSÉUM PARIS | Rio Janeiro | d'Orbigny] [LECTOTYPE ঐ) Anomalochilus singularis | Blanchard, $1850 \mid$ des. J. Fuhrmann \& | F.Z. Vaz-de-Mello, 2014].

\section{Paralectotypes}

BRAZIL: $2 \widehat{\jmath} \widehat{\jmath}$, MNHN, labels: [MUSÉUM PARIS | d'Orbigny] [PARALECTOTYPE $\widehat{\jmath}$ | Anomalochilus singularis | Blanchard, $1850 \mid$ des. J. Fuhrmann \& | F.Z. Vaz-de-Mello, 2014].

Plectris tomentosa LePeletier de Saint-Fargeau \& Audinet-Serville, 1828

Figs 4A, 20G-H

Plectris tomentosa LePeletier de Saint-Fargeau \& Audinet-Serville, 1828: 369. 


\section{Note}

Plectris tomentosa was described based on an undetermined number of specimens.

\section{Material examined}

Lectotype (here designated, Fig. 20G-H)

BRAZIL: $\widehat{\jmath}$, MNHN, labels: [MUSÉUM PARIS] [P. tomentosa | Lep. St. Farg. et Serv. | Brésil] [LECTOTYPE | Plectris tomentosa | LePeletier \& | Audinet-Serville, $1828 \mid$ des. J. Fuhrmann \& | F.Z. Vaz-de-Mello, 2014].

\section{Remarks}

A specimen at BMNH is labeled as Frey's neotype for P. tomentosa (Fig. 22F), but a neotype for this species was never proposed.

\section{Pseudoserica Guérin-Méneville, 1838, revalidated}

Pseudoserica Guérin-Méneville, 1838: 86.

Gama Blanchard, 1850: 118, syn. nov. Type species: Gama grandicornis Blanchard, 1850 (designation: Lacordaire 1856: 265).

Harpodactyla Burmeister, 1855: 17, syn. nov. Type species: Harpodactyla grandicornis Burmeister, 1855 (designation: Evans 2003: 268).

Pachylotoma Blanchard, 1850: 121, syn. nov. Type species: Pachylotoma viridis Blanchard, 1850 (designation: monotypy).

Pseudoserica - Blanchard 1850: 128 (synonymy with Plectris).

Harpodactyla - Gemminger \& Harold 1869: 1411 (synonymy with Gama).

Pachylotoma - Katovich 2008: 6 (synonymy with Gama).

\section{Type species}

Serica marmorea Guérin-Méneville, 1831 (designation: monotypy).

\section{Diagnosis}

Clypeal posterior angle partially covering the canthus (Fig. 13A); pronotal anterior margin beaded, posterior margin not beaded and with two acute projections over the anterior elytron-scutellum contact, posterior angle acute (Fig. 13A); prosternum anteriorly concave (similar to Fig. 12K) or an anterior longitudinal carina; protibia with 1-3 external teeth and with or without spur; elytron with posterior margin not beaded; male mesotarsomere I with an internal hook-like spine (Fig. 13B); abdomen with intersegmental membrane VII-VIII concealed.

\section{Remarks}

The genus is recognized by having the posterior pronotal margin with two lateromedial teeth which project over the anterior elytron-scutellum contact. In addition, the male mesotarsomere I has a hooklike spine (Fig. 13B), that also occurs in Mallotarsus (Fig. 13C) and part of Dicrania (e.g., D. ebenina).

The present study revalidates Pseudoserica, formerly a synonym of Plectris, based on the following characters (opposition to Plectris): posterior pronotal margin with two lateromedial teeth over the anterior elytron-scutellum contact (margin straight); male mesotarsomere I with a hook-like spine (without spines); internal area of metatarsomere $\mathrm{V}$ without spine-like setae (with spine-like setae). 
Pseudoserica grandicornis (Blanchard, 1850) comb. nov.

Fig. 20I-J

Gama grandicornis Blanchard, 1850: 118.

Harpodactyla grandicornis Burmeister, 1855: 18 (different species, with the same epithet, later synonymized).

Harpodactyla grandicornis - Gemminger \& Harold 1869: 1411 (synonymy).

Note

Gama grandicornis was described based on an undetermined number of specimens.

\section{Material examined}

Lectotype (here designated, Fig. 20I-J)

BRAZIL: $\widehat{\lambda}$, MNHN, labels: [11 | 44] [MUSÉUM PARIS | Rio | de Castelnau] [Gama | grandicornis, B1.] [Gama | grandicornis | Blanchard | fide K.Katovich | 03] [LECTOTYPE $\sigma^{\lambda}$ | Gama grandicornis | Blanchard, 1850 | des. J. Fuhrmann \& | F.Z. Vaz-de-Mello, 2014] [Pseudoserica | grandicornis | (Blanchard, 1850) §ै | des. J. Fuhrmann \& | F.Z. Vaz-de-Mello, 2014].

\section{Paralectotypes $(\mathrm{n}=3)$}

BRAZIL: $2 \hat{\jmath}$ ô, MNHN, labels: [11 | 44] [MUSÉUM PARIS | Rio | de Castelnau] [PARALECTOTYPE § | Gama grandicornis | Blanchard, 1850| des. J. Fuhrmann \& | F.Z. Vaz-de-Mello, 2014] [Pseudoserica | grandicornis $\mid$ (Blanchard, 1850) $\hat{\sigma} \mid$ des. J. Fuhrmann \& |F.Z. Vaz-de-Mello, 2014]; 1 q, BMNH, labels: [Co- | type] [11 | 44] [MUSÉUM PARIS | Rio | de Castelnau] [1946.181] [Gama | grandicornis Bl. | R. Paulian det.] [PARALECTOTYPE $q$ | Gama grandicornis | Blanchard, 1850 | des. J. Fuhrmann \& | F.Z. Vaz-de-Mello, 2014] [Pseudoserica | grandicornis | (Blanchard, 1850) $q$ | des. J. Fuhrmann \& | F.Z. Vaz-de-Mello, 2014].

Pseudoserica marmorea (Guérin-Méneville, 1831)

Figs 13A-B, D-E, 20M-N

Serica marmorea Guérin-Méneville, 1831: fig. 3.

Pseudoserica marmorea - Guérin-Méneville 1838: 86.

Plectris marmorea - Blanchard 1850: 128 (Pseudoserica synonym of Plectris).

\section{Note}

Serica marmorea was described based on an undetermined number of specimens.

\section{Material examined}

Lectotype (here designated, Fig. 20M-N)

BRAZIL: + , MNHN, labels: [Sta. Cather. | Durville] [MUSÉUM PARIS] [Pseudoserica | marmorea | Guer.] [Plectris $\mid$ marmorea | Blanch.] [Typus] [P. marmorea | Guerin | Brésil] [LECTOTYPE o | Serica marmorea | Guérin-Méneville, 1831 | des. J. Fuhrmann \& | F.Z. Vaz-de-Mello, 2014] [Pseudoserica marmorea $\mid$ (Guérin-Méneville, 1831) | + | det. J. Fuhrmann \& | F.Z. Vaz-de-Mello, 2014].

Pseudoserica viridis (Blanchard, 1850) comb. nov.

Fig. 20K-L

Pachylotoma viridis Blanchard, 1850: 121.

Gama viridis - Katovich 2008: 6 (Pachylotoma synonym of Gama). 


\section{Note}

Pachylotoma viridis was described based on an undetermined number of specimens.

\section{Material examined}

Lectotype (here designated, Fig. 20K-L)

SOUTH AMERICA: 1 ô, MNHN, labels: [MUSÉUM PARIS] [Pachylotoma | viridis | Blanchard | fide K. Katovich 03] [LECTOTYPE $\precsim \mid$ Pachylotoma viridis | Blanchard, $1850 \mid$ des. J. Fuhrmann \& | F.Z. Vaz-de-Mello, 2014] [Pseudoserica viridis | (Blanchard, 1850) §ै| des. J. Fuhrmann \& | F.Z. Vaz-deMello, 2014].

\section{Rhinaspis Perty, 1833}

Mallogaster Dejean, 1833: 162, nomen nudum.

Rhinaspoides Moser, 1919b: 348. Type species: Rhinaspoides aeneofusca Moser, 1919 (designation: monotypy).

Ulomenes Blanchard, 1850: 125. Type species: Ulomenes hypocrita Blanchard, 1850 (designation: monotypy); synonym of Rhinaspis fuhrmanni Smith (2016: 2) (replacement name).

Hyporhiza Dejean, 1833: 162. Type species: Melolontha hypocrita Mannerheim, 1829 (designation: monotypy).

Rhinaspoides - Katovich 2008: 6 (synonymy).

Ulomenes - Katovich 2008: 6 (synonymy).

Hyporhiza - Bousquet \& Bouchard 2013: 37 (synonymy).

\section{Type species}

Rhinaspis schrankii Perty, 1833 (designation: monotypy); junior synonym of Rhinaspis aenea (Billberg, 1820).

\section{Diagnosis}

Clypeal ventral area strongly widened, posterior angle partially covering the canthus (Fig. 10D-F); pronotal anterior margin beaded, lateral margins crenulate, posterior margin prominent (Fig. 10D-F); prosternum anteriorly concave (similar to Fig. 12K); protibia with three external teeth and with a spur; elytron with three smooth and weakly prominent lines between internal margin and humerus, posterior and posterointernal margins not beaded; abdomen with intersegmental membrane VII-VIII concealed.

\section{Remarks}

The majority of species of Rhinaspis are characterized by having the male clypeus anteriorly prominent with a broad, truncate apex (e.g., Rhinaspis aenea, Fig. 10D) or a bifid horn (e.g., Rhinaspis ohausi Moser, 1921, Fig. 10E), and male and female clypeal posterior angle acute and strongly extended over the canthus. However, some species have a trapezoid or rectangular clypeus (e.g., Rhinaspis aeneofusca, Fig. 10F). The structure of the pronotum and the elytron, described in "diagnosis", are more suitable to distinguish Rhinaspis from other Macrodactylini genera.

\section{Rhinaspis aeneofusca (Moser, 1919)}

Figs 10F-G, J-K, 20O-P

Rhinaspoides aeneofusca Moser, 1919: 348.

Rhinaspis aeneofusca - Katovich 2008: 6 (Rhinaspoides synonym of Rhinaspis). 


\section{Note}

Rhinaspoides aeneofusca was described (Moser 1919b) based on an undetermined number of males and females.

\section{Material examined}

Lectotype (here designated, Fig. 20O-P)

BRAZIL: $\sigma^{\lambda}$, ZMHU, labels: [Santa Leopoldina | Esp. Santo] [Rhinaspoides | aeneofusca | Type Mos.] [LECTOTYPE | Rhinaspoides | aeneofusca | Moser, $1919 \hat{\diamond} \mid$ des. J. Fuhrmann \& | F.Z. Vaz de Mello 2014]

\section{Paralectotypes $(n=4)$}

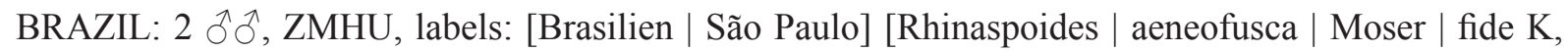
Katovich 02] [PARALECTOTYPE | Rhinaspoides | aeneofusca $\mid$ Moser, 1919 ঐ | des. J. Fuhrmann \& | F.Z. Vaz de Mello 2014]; 1 \%, ZMHU, labels: [Rhinaspoides | aeneofusca | Type Mos.] [Brasilien | São Paulo] [PARALECTOTYPE | Rhinaspoides $\mid$ aeneofusca $\mid$ Moser, 1919 q $\mid$ des. J. Fuhrmann \& | F.Z. Vaz de Mello 2014]; 1 q, ZMHU, labels: [Brasilien | São Paulo] [PARALECTOTYPE | Rhinaspoides | aeneofusca | Moser, 1919 \& | des. J. Fuhrmann \& | F.Z. Vaz de Mello 2014].

Rhinaspis fuhrmanni Smith, 2016

Fig. 21A-B

Ulomenes hypocrita Blanchard, 1850: 125.

Rhinaspis fuhrmanni Smith, 2016: 2 (replacement name).

Rhinaspis hypocrita - Katovich 2008: 6 (Ulomenes synonym of Rhinaspis).

\section{Note}

Ulomenes hypocrita was described based on an undetermined number of specimens.

\section{Material examined}

Lectotype (here designated, Fig. 21A-B)

BRAZIL: + , MNHN, labels: [9 | 43] [Ulomenes | hypocrita, B1.] [MUSÉUM PARIS | Brésil | Clauneu] [Ulomenes | hypocrita | Blanchard | fide K. Katovich 03] [LECTOTYPE | Ulomenes hypocrita | Blanchard, 1850 ㅇ | des. Fuhrmann \& | F.Z. Vaz-de-Mello, 2014].

\section{Remarks}

Smith (2016) erected Rhinaspis fuhrmanni as a replacement name for Ulomenes hypocrita Blanchard (type species of Ulomenes) to avoid a secondary homonym with Rhinaspis hypocrita (Mannerheim, 1829) (described as Melolontha hypocrita, and type species of Hyporhiza).

\section{Schizochelus Blanchard, 1850}

Gastrohoplus Moser, 1921: 165, syn. nov. Type species: Gastrohoplus mirabilis Moser, 1921 (designation: monotypy).

\section{Type species}

Schizochelus flavescens Blanchard, 1850 (designation: Evans 2003: 346). 


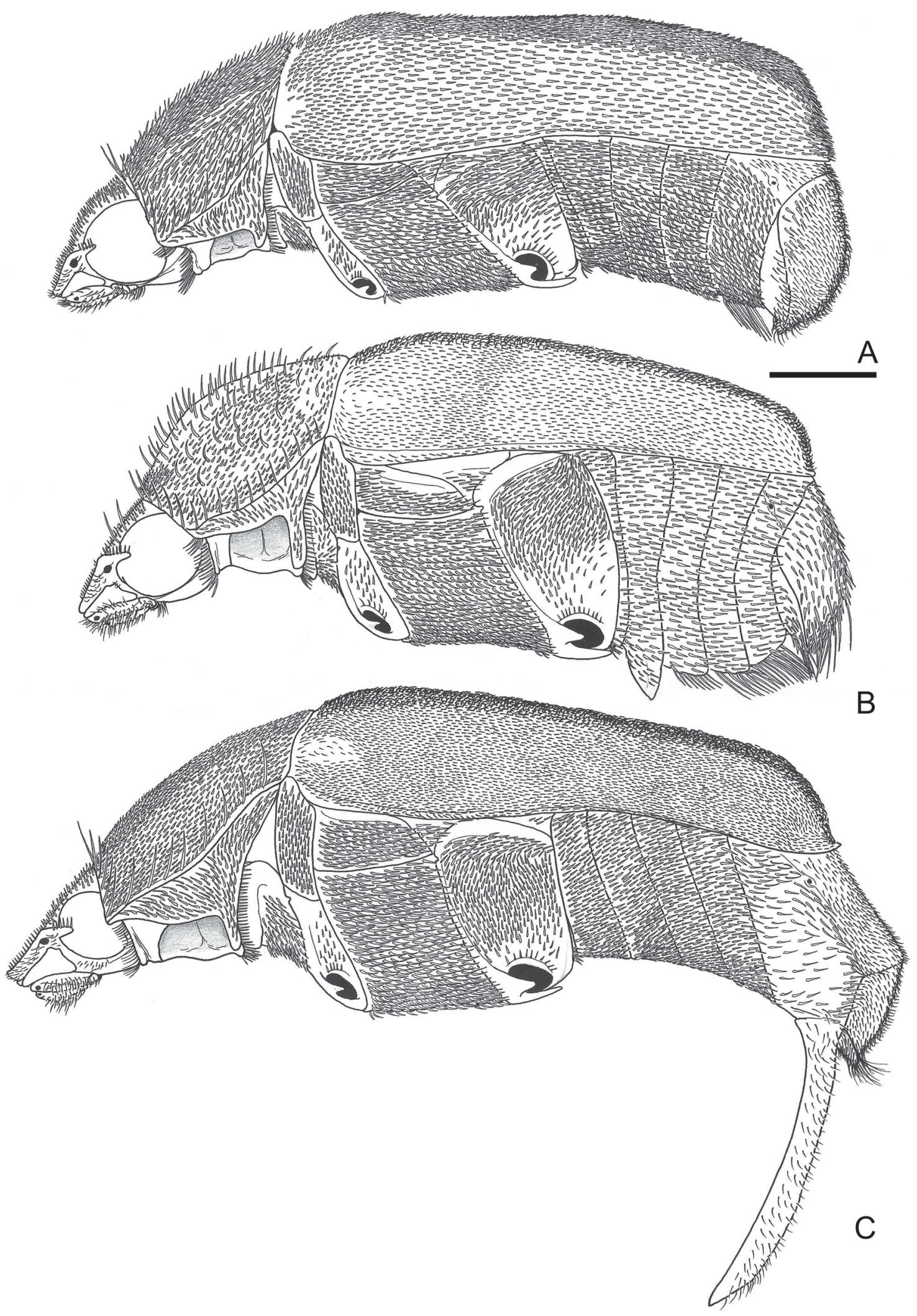

Fig. 15. Schizochelus Blanchard, 1850, ô, habitus, lateral (without some appendages). A. Schizochelus flavescens Blanchard, 1850. B. Schizochelus bicoloripes Blanchard, 1850. C. Schizochelus mirabilis (Moser, 1921) comb. nov. Scale bar $=1 \mathrm{~mm}$. 


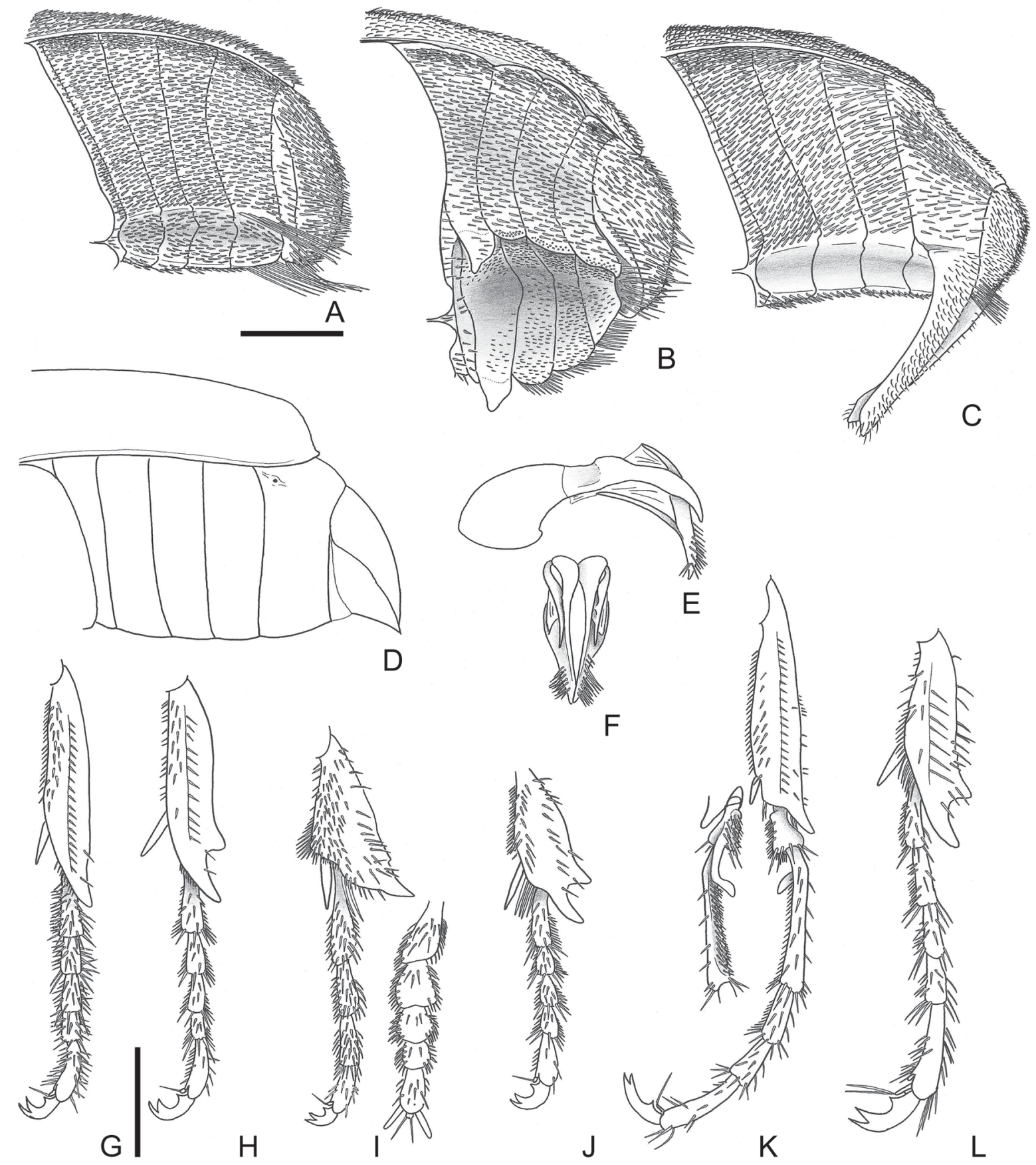

Fig. 16. Schizochelus Blanchard, 1850. A-C. Male abdomen, lateroventral. D. Female abdomen, lateral. E-F. Aedeagus (lateral, parameres apex). G-L. Protibia-tarsus (male, female) (with detail of tarsus: I = dorsal view; K = ventral view). A, E-H. Schizochelus flavescens Blanchard, 1850. B, I-J. Schizochelus bicoloripes Blanchard, 1850. C-D, K-L. Schizochelus mirabilis (Moser, 1921) comb. nov. Scale bars $=1 \mathrm{~mm}$. 

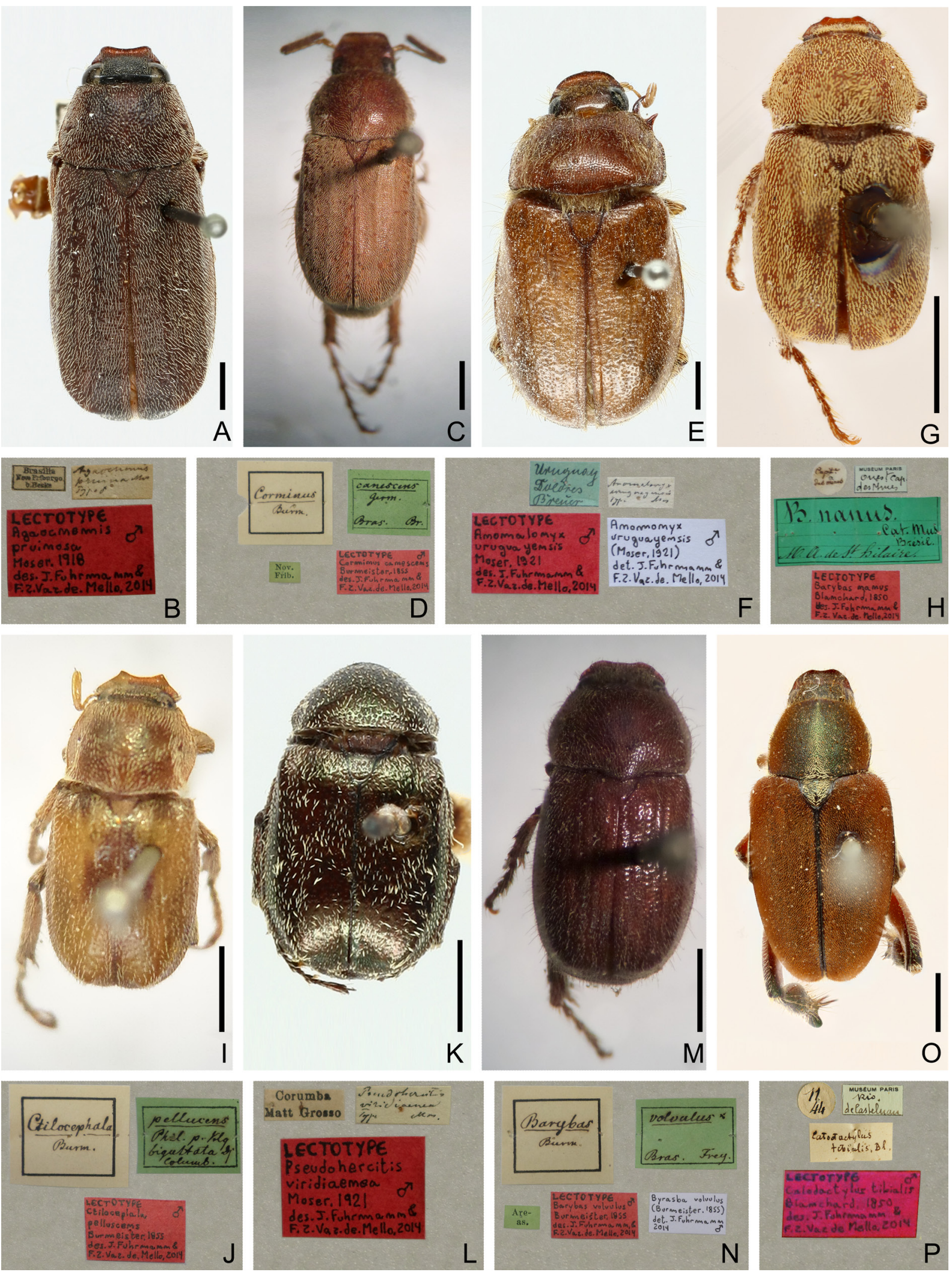

Fig 17. Lectotypes (dorsal, labels). A-B. Agaocnemis pruina Moser, 1918. C-D. Corminus canescens Burmeister, 1855. E-F. Anomalonyx uruguayensis Moser, 1921. G-H. Barybas nana Blanchard, 1850. I-J. Ctilocephala pelluscens Burmeister, 1855. K-L. Pseudohercitis viridiaenea Moser, 1921. M-N. Barybas volvulus Burmeister, 1855. O-P. Calodactylus tibialis Blanchard, 1850. Scale bars $=2 \mathrm{~mm}$. 

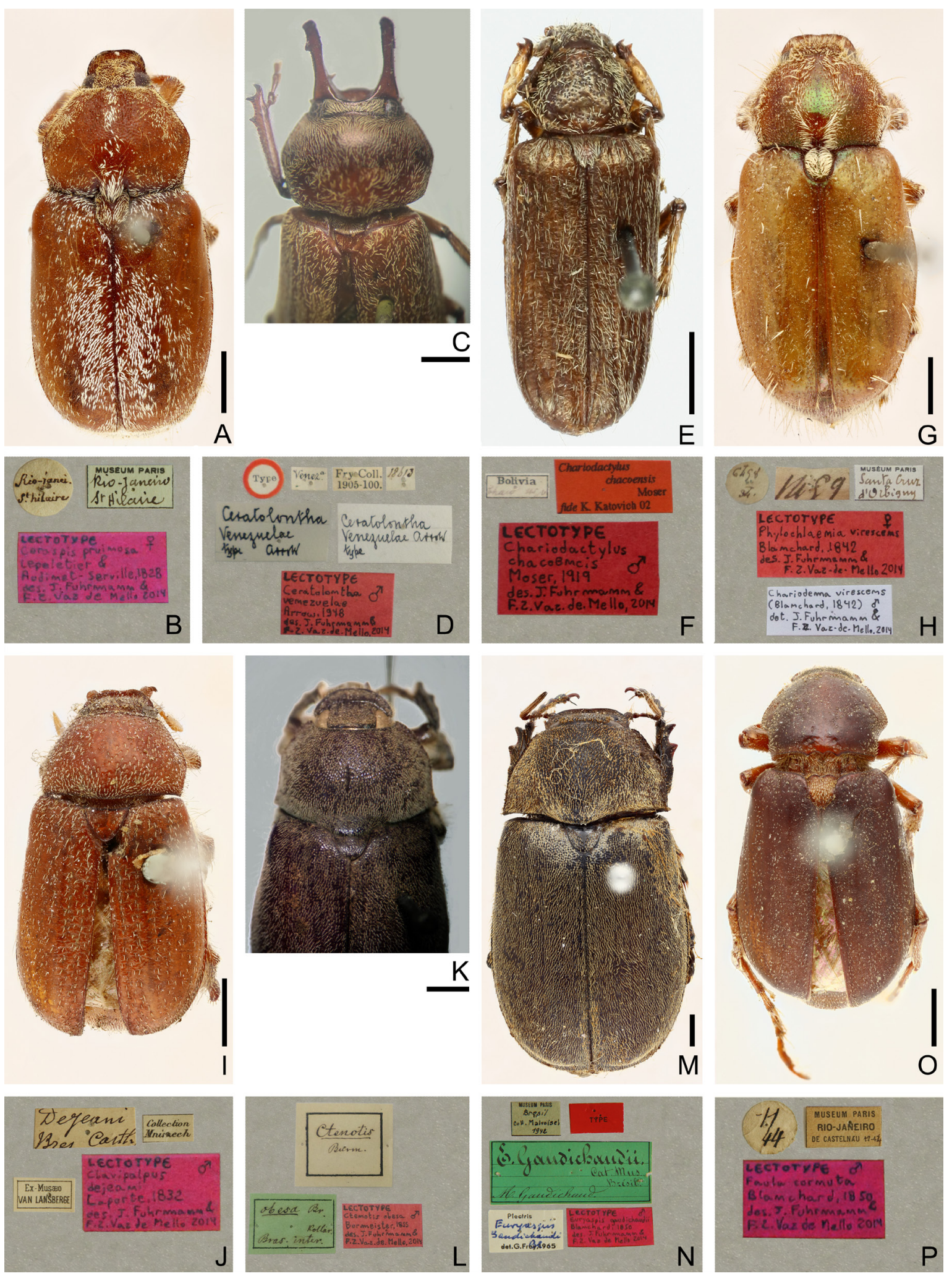

Fig 18. Lectotypes (dorsal, labels). A-B. Ceraspis pruinosa LePeletier de Saint-Fargeau \& AudinetServille, 1828. C-D. Ceratolontha venezuelae Arrow, 1948. E-F. Chariodactylus chacoensis Moser, 1919. G-H. Philochlaenia virescens Blanchard, 1842. I-J. Clavipalpus dejeani Laporte, 1832. K-L. Ctenotis obesa Burmeister, 1855. M-N. Euryaspis gaudichaudii Blanchard, 1850. O-P. Faula cornuta Blanchard, 1850. Scale bars $=2 \mathrm{~mm}$. 

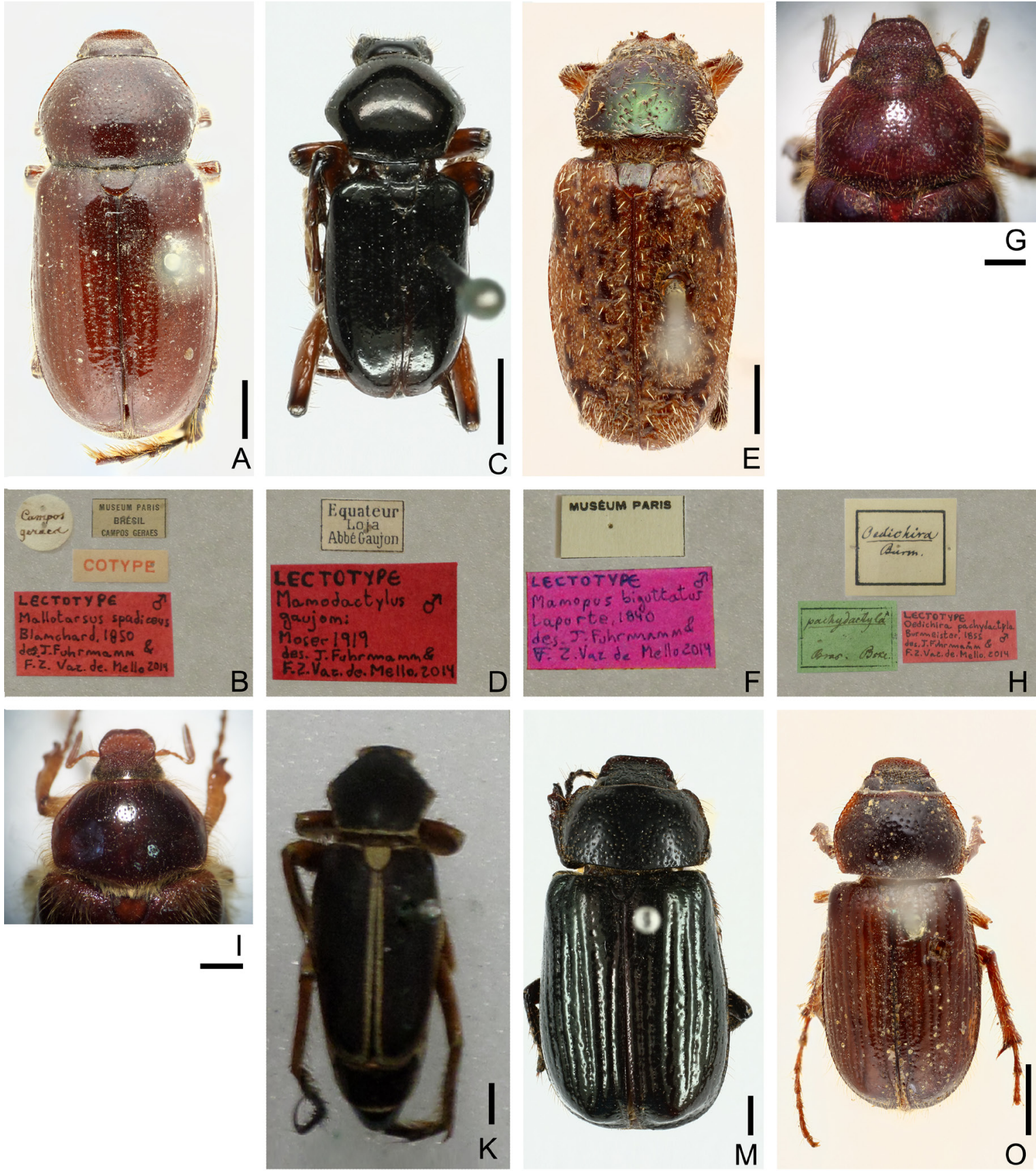

$\mathrm{H}$
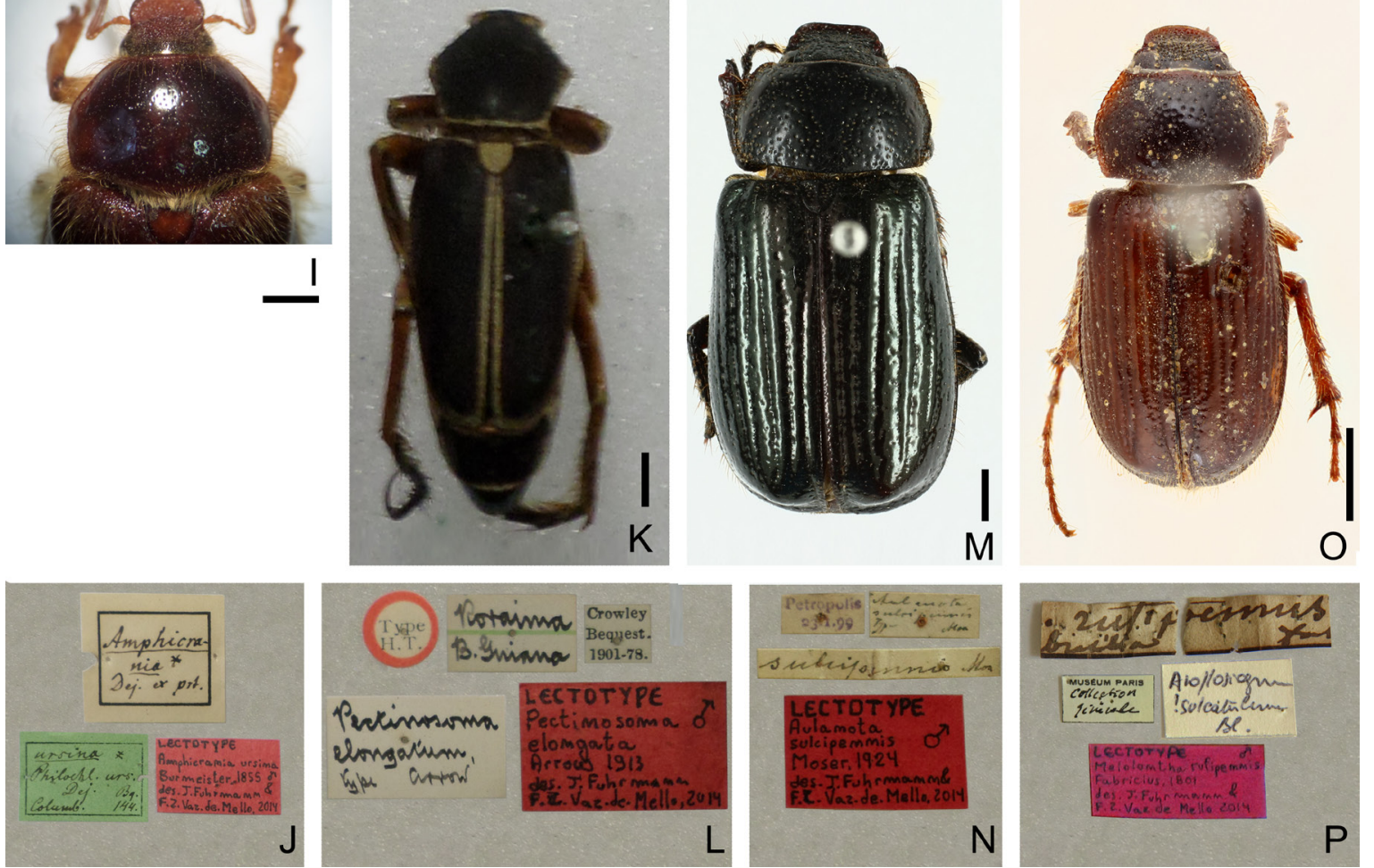

Fig 19. Lectotypes (dorsal, labels). A-B. Mallotarsus spadiceus Blanchard, 1850. C-D. Manodactylus gaujoni Moser, 1919. E-F. Manopus biguttatus Conte de Castelnau, 1840. G-H. Oedichira pachydactyla Burmeister, 1855. I-J. Amphicrania ursina Burmeister, 1855. K-L. Pectinosoma elongata Arrow, 1913. M-N. Aulanota sulcipennis Moser, 1924. O-P. Melolontha rufipennis Fabricius, 1801. Scale bars $=2 \mathrm{~mm}$. 

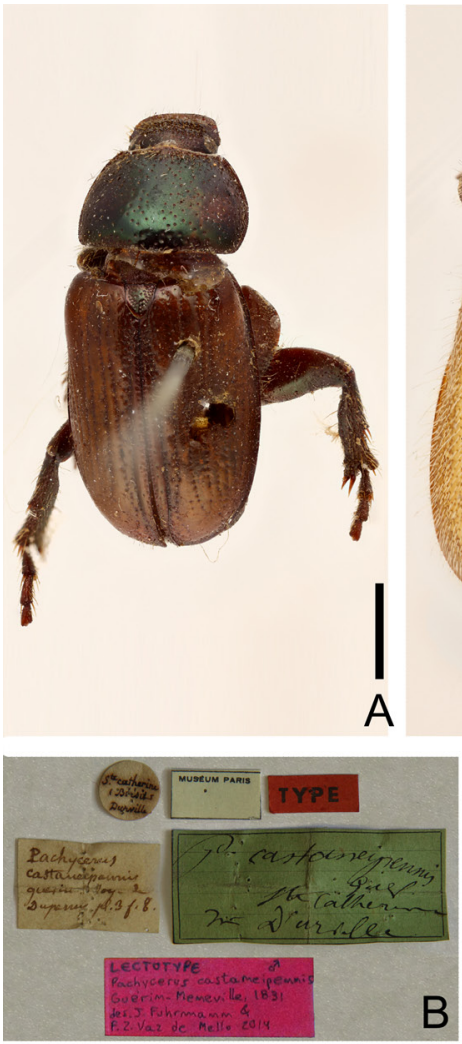

B

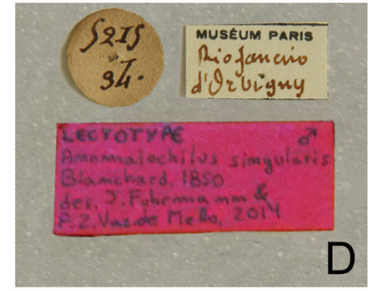

D
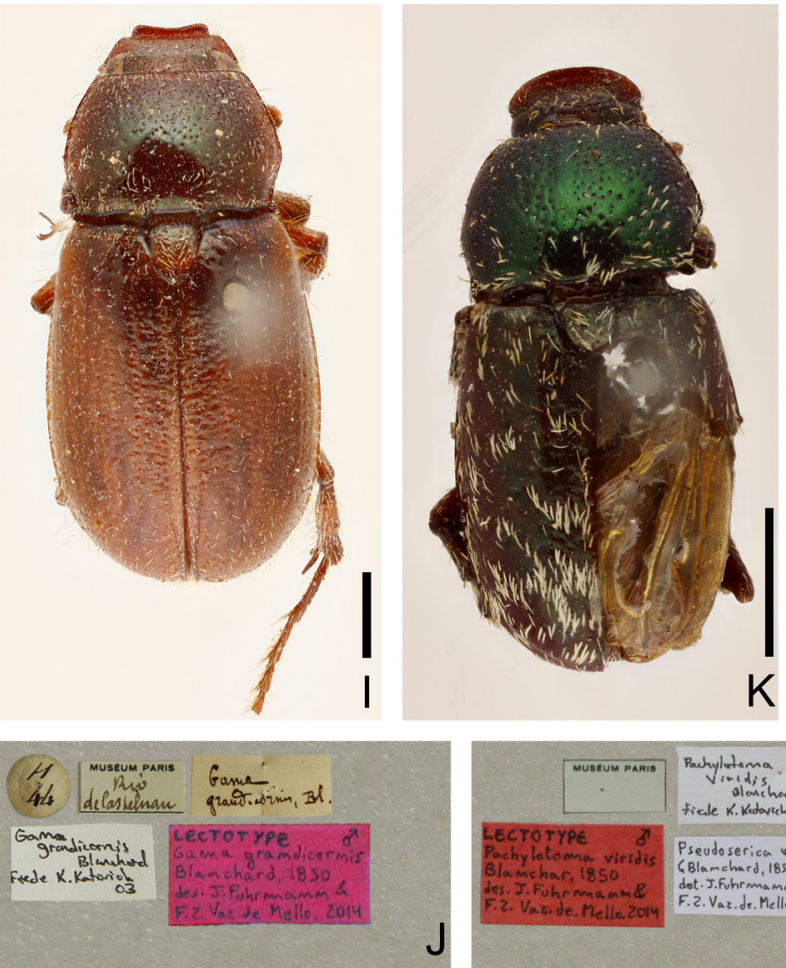
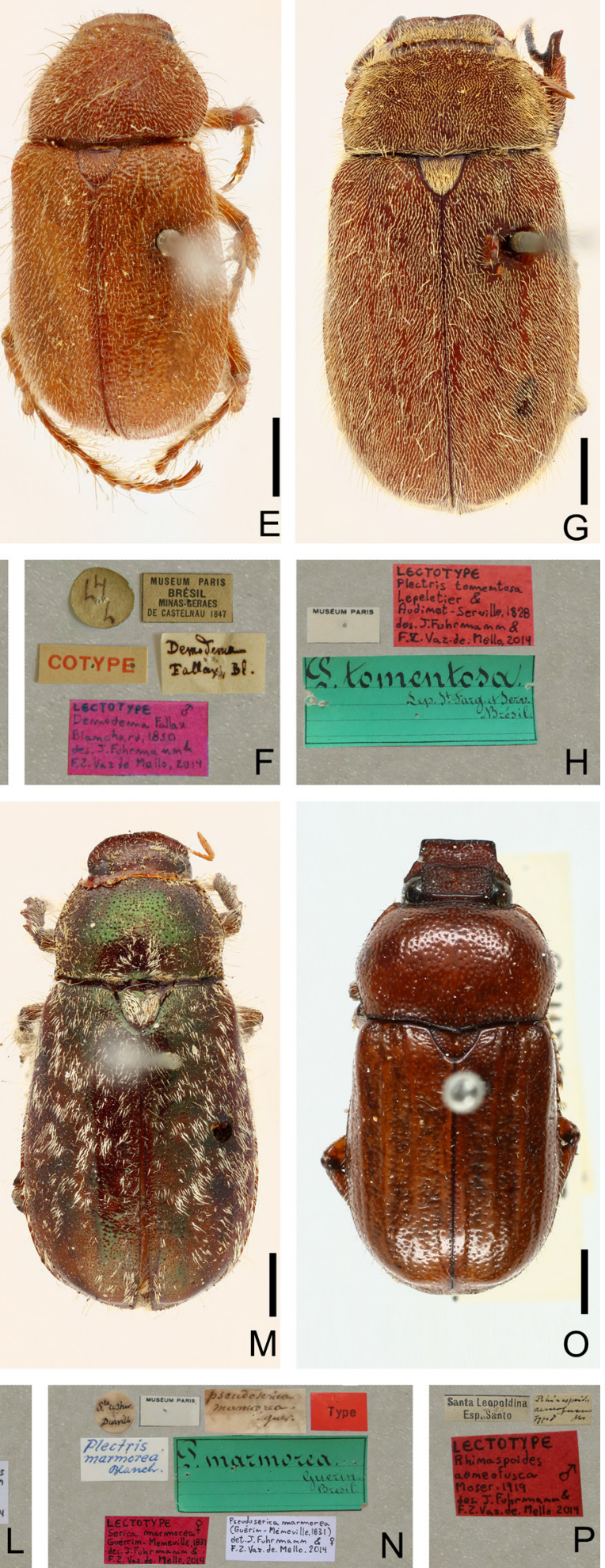

Fig 20. Lectotypes (dorsal, labels). A-B. Pachycerus castaneipennis Guérin-Méneville, 1831. C-D. Anomalochilus singularis Blanchard, 1850. E-F. Demodema fallax Blanchard, 1850. G-H. Plectris tomentosa LePeletier de Saint-Fargeau \& Audinet-Serville, 1828. I-J. Gama grandicornis Blanchard, 1850. K-L. Pachylotoma viridis Blanchard, 1850. M-N. Serica marmorea Guérin-Méneville, 1831. O-P. Rhinaspoides aeneofusca Moser, 1919. Scale bars $=2 \mathrm{~mm}$. 

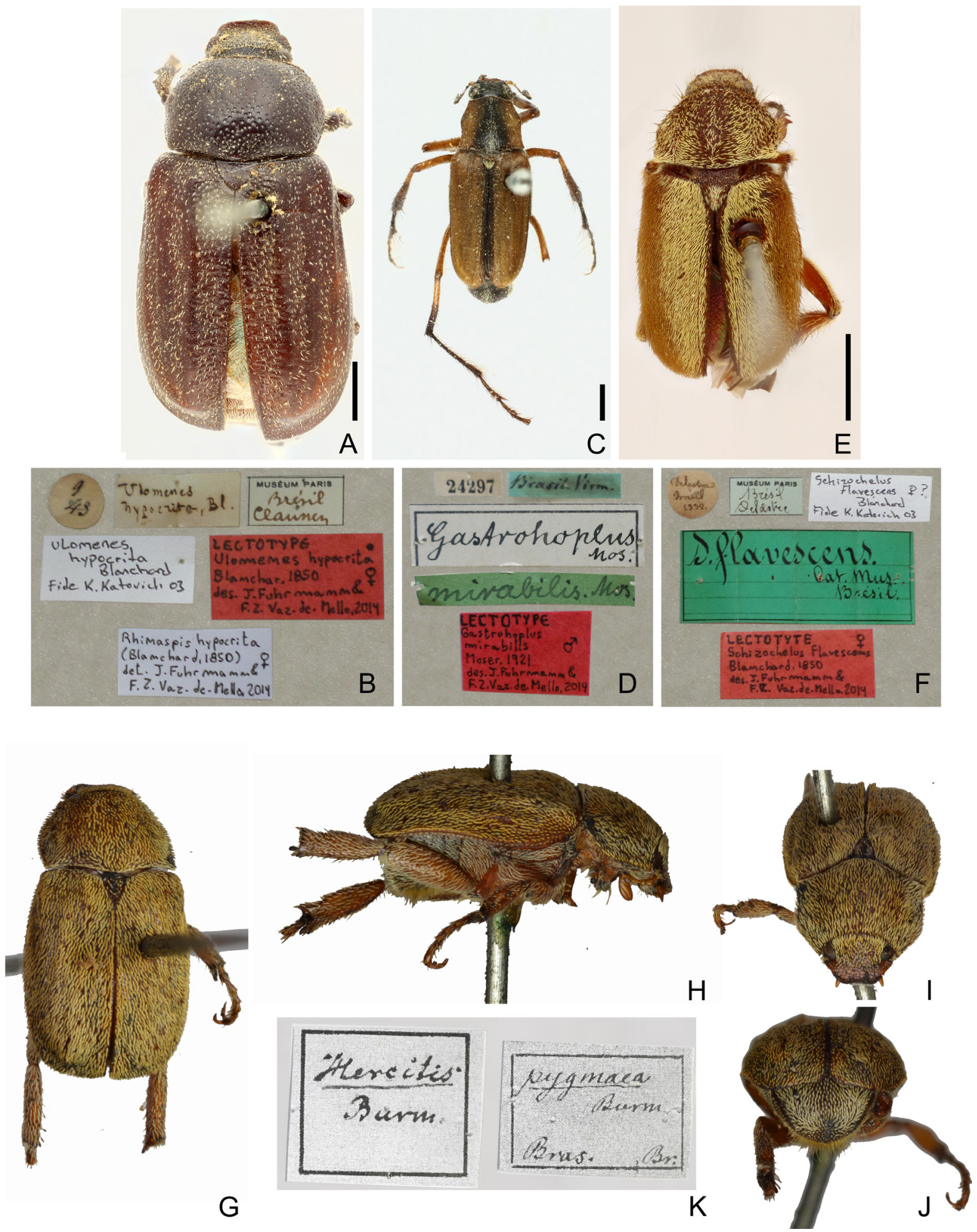

Fig 21. Primary types. A-F. Lectotypes (dorsal, labels). A-B. Ulomenes hypocrita Blanchard, 1850. C-D. Gastrohoplus mirabilis Moser, 1921. E-F. Schizochelus flavescens Blanchard, 1850. G-K. Syntype of Hercitis pygmaea Burmeister, 1855 (by Holger Dombrow). G. Dorsal. H. Lateral. I. Frontal. J. Posterior. K. Labels. Scale bars: A, C, E $=2 \mathrm{~mm}$; G-J without scale (specimen about 4-4.2 $\mathrm{mm}$ according to original description). 


\section{Diagnosis}

Clypeal anteroventral area strongly reduced (Fig. 15, similar to Fig. 11H); pronotal anterior and posterior margins not beaded; prosternum with an anterior longitudinal carina; protibia with 1-2 external teeth (male with tooth II absent or reduced) and with a spur (Fig. 16G-L); male metatibia without spur; male ventrites I-V longitudinally concave (Fig. 16A-C); female abdomen sinuous in lateral view (Fig. 16D); tarsal claws with a proximal tooth (Fig. 16G-L).

\section{Remarks}

The genera Isonychus and Schizochelus are similar to each other. Schizochelus is distinguished as follows (opposition to Isonychus): male metatibial spur absent (metatibia with 1-2 spurs); male ventrites I-V with medial concavity (Fig. 16A-C) (without), male ventrite VI straight and with short and sparse setae (straight or laterally enlarged, and with or without lateral comb of long setae); female abdomen sinuous in lateral view (Fig. 16D) (convex).

The present study proposes the synonymy of Gastrohoplus with Schizochelus, see chapter on "Male morphology and nomenclature" (below) for more information about the new synonymy.
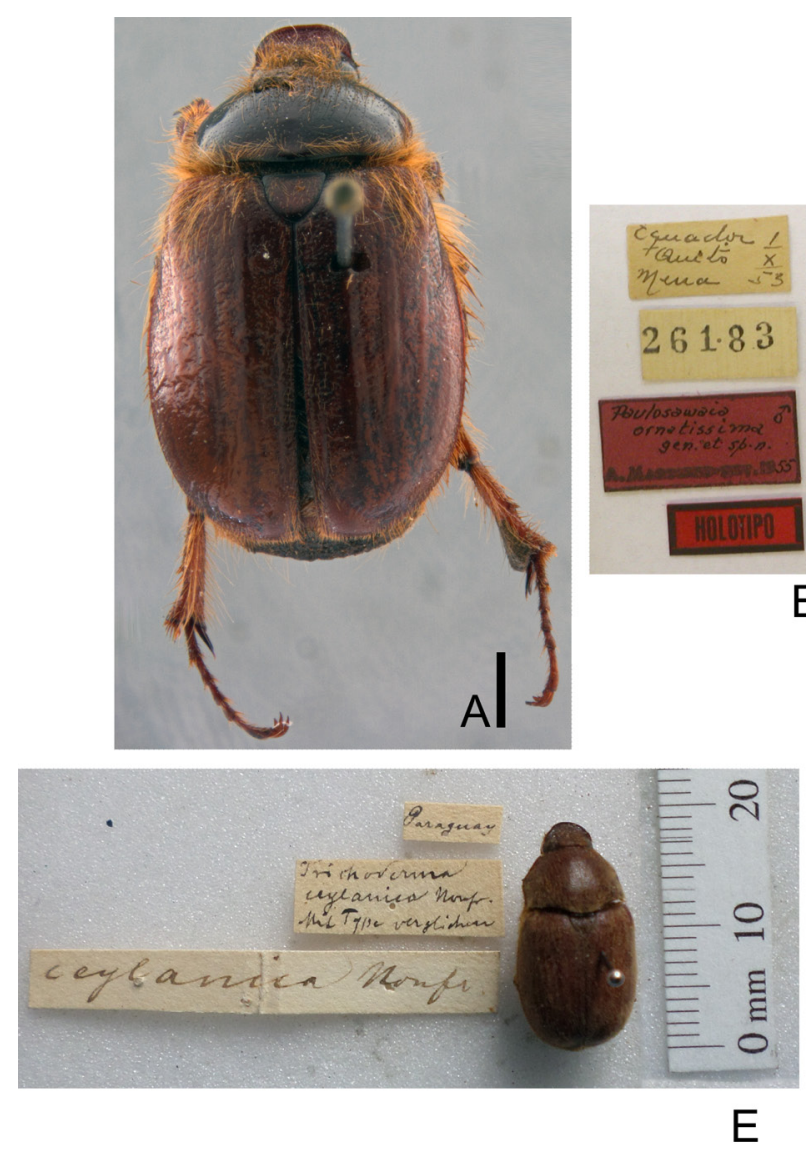
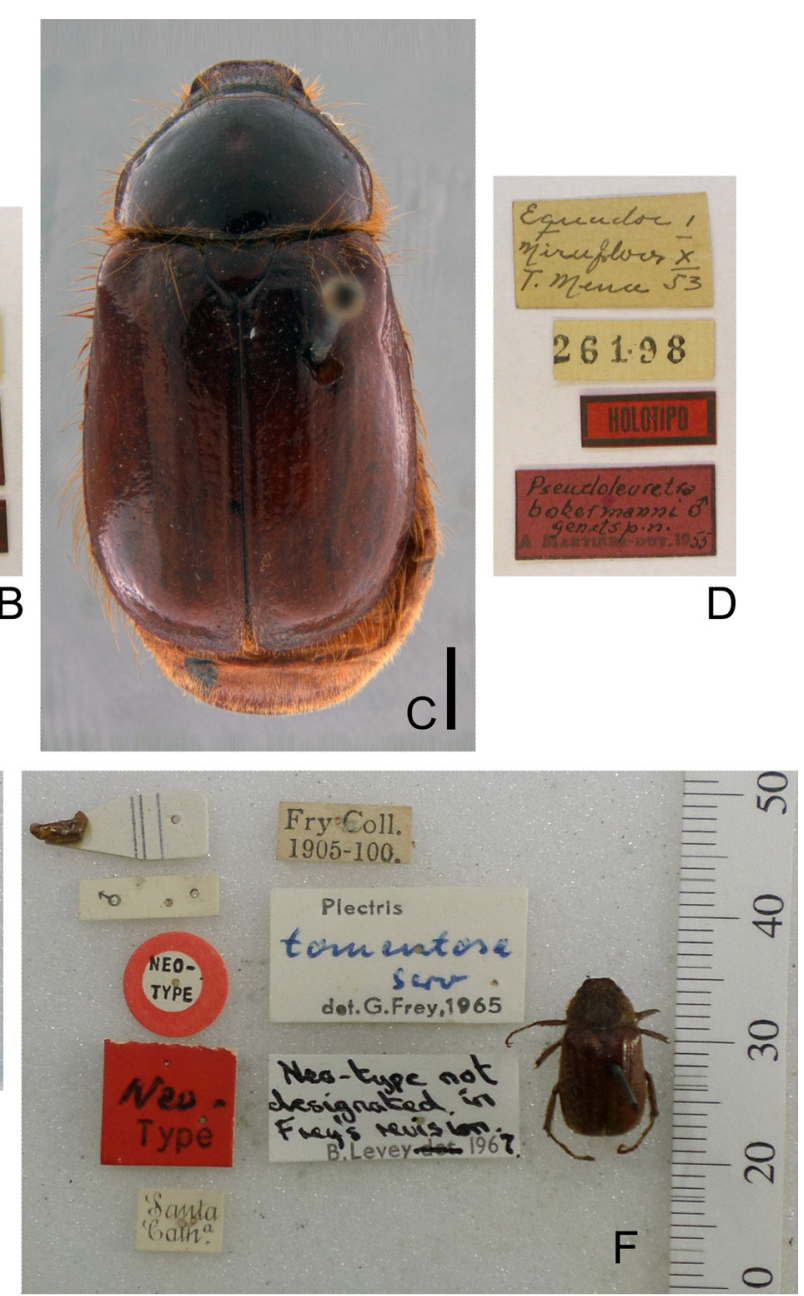

Fig. 22. A-D. Holotype, $\widehat{\partial}$ (dorsal, labels). A-B. Paulosawaya ornatissima Martínez \& d'Andretta, 1956. C-F. Pseudoleuretra bokermanni Martínez \& d'Andretta, 1956. E-F. Non-type material. E. Junkia ceylanica Nonfried, 1894. F. Plectris tomentosa LePeletier de Saint-Fargeau \& Audinet-Serville, 1828. Scale bars $=2 \mathrm{~mm}$. 
Schizochelus flavescens Blanchard, 1850

Figs 15A, 16A, E-H, 21E-F

Schizochelus flavescens Blanchard, 1850: 89.

Note

Schizochelus flavescens was described based on an undetermined number of specimens.

\section{Material examined}

Lectotype (here designated, Fig. 21E-F)

BRAZIL: + , MNHN, labels: [Delastre |Brasil | 1832] [MUSÉUM PARIS |Brésil |Delastre] [S. flavescens | Cat. Mus. | Brésil] [Schizochelus | flavescens | Blanchard | $q$ ? | fide K. Katovich 03] [LECTOTYPE $q$ | Schizochelus flavescens | Blanchard 1850 | des. J. Fuhrmann \& | F.Z. Vaz de Mello 2014].

Schizochelus mirabilis (Moser, 1921) comb. nov.

Figs $15 \mathrm{C}, 16 \mathrm{C}-\mathrm{D}, \mathrm{K}-\mathrm{L}, 21 \mathrm{C}-\mathrm{D}$

Gastrohoplus mirabilis Moser, 1921: 165.

\section{Note}

Gastrohoplus mirabilis was described based on an undetermined number of males.

\section{Material examined}

Lectotype (here designated, Fig. 21C-D)

BRAZIL: $\widehat{O}$, ZMHU, labels: [24297] [Brasil Virm.] [Gasprohophus | Mos.] [mirabilis Mos.] [LECTOTYPE | Gastrohoplus | mirabilis | Moser, $1921 \mid$ ठ $\mid$ des. J. Fuhrmann \& | F.Z. Vaz-de-Mello, 2014].

\section{Key to genera of Macrodactylini}

1. Internal area of metatarsomere $\mathrm{V}$ with spine-like setae and/or a proximal tubercle or raised carina (Fig. 4A-D)

- Internal area of metatarsomere V unarmed and never with differentiated spine-like setae, a dense setal comb sometimes present

2. Metatarsus with one claw and without empodium Astaenoplia Martínez, 1957

- Metatarsus with two claws and empodium .3

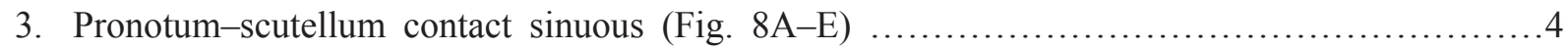

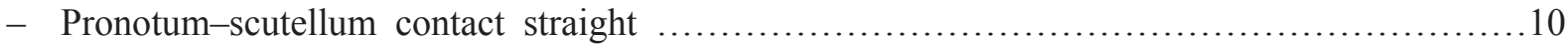

4. Metacoxae subcontiguous .........Ceraspis LePeletier de Saint-Fargeau \& Audinet-Serville, 1828

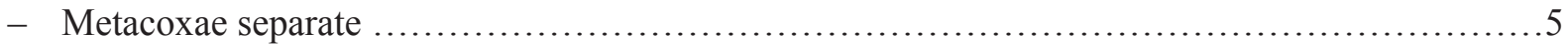

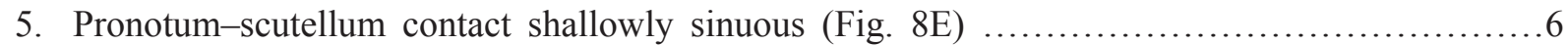

- Pronotum-scutellum contact deeply sinuous (Fig. 8A-D) ................................

6. Prosternal posterior process raised and bifid; protibial internal angle rounded, female protibia with a spur; male ventrite $\mathrm{V}$ with medial lobe bearing truncate setae in a transverse palisade (Fig. 14D-E)

Pectinosoma Arrow, 1913

- Prosternal posterior process not prominent and rounded; protibial internal angle acute (Fig. 8F), female protibial spur absent; male ventrite $\mathrm{V}$ unarmed 
7. Male ventrite I with a medial spine (Fig. 14A); female pygidial anterior margin sinuous (Fig. 14B-C) Ancistrosoma Curtis, 1835

- Male ventrite I unarmed; female pygidial anterior margin straight .8

8. Pronotum-scutellum contact acutely sinuous (Fig. 8B) Chariodema Blanchard, 1850

- Pronotum-scutellum contact roundly sinuous (Fig. 8D); scutellum cordate

9. Pronotal posterior angle acutely raised (similar to Fig. 8B); protibia with two external teeth; total length usually less than $20 \mathrm{~mm}$

Pseudopectinosoma Katovich, 2011

- Pronotal posterior angle obtuse or rounded; protibia with 3-4 external teeth; total length usually greater than $20 \mathrm{~mm}$ Faula Blanchard, 1850

10. Pronotal lateral margins crenulate or serrate; mesoscutum with a transverse carina (Fig. 6G); male protarsomere I with an internodistal tooth (Fig. 5A-B)

- Pronotal lateral margins straight; mesoscutum without carina; protarsomere I with or without a tooth

11. Protibia with three external teeth and with a spur; male metatarsomere I with internal hook-like tooth (Fig. 13C); metafemur with row of spine-like setae (Fig. 13H) ...Mallotarsus Blanchard, 1850

- Protibia with 1-3 external teeth and with or without a spur; male metatarsomere I unarmed;

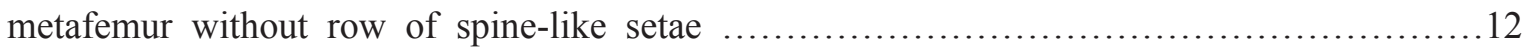

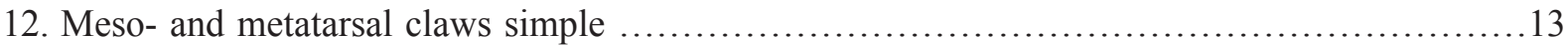

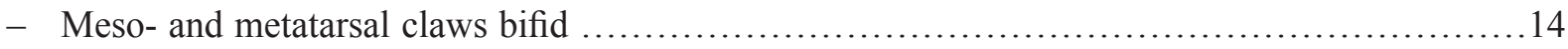

13. Dorsum usually with scale-like setae; meso- and metatibiae with an evident medial

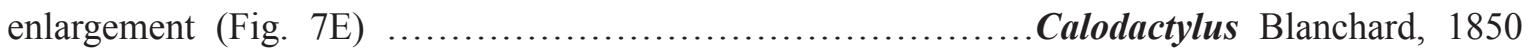

- Dorsum never with scale-like setae; meso- and metatibiae distally parallel Dasyus LePeletier de Saint-Fargeau \& Audinet-Serville, 1828

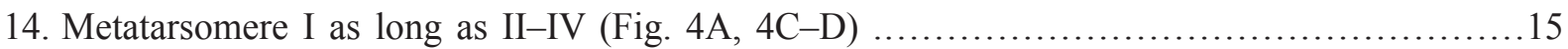

- Metatarsomere I as long as or shorter than II-III (Fig. 4B) ............................... 16

15. Metatarsomere II wider than long (Fig. 4C-D) Oedichira Burmeister, 1855

- Metatarsomere II at least twice as long as wide (Fig. 4A)

Plectris LePeletier de Saint-Fargeau \& Audinet-Serville, 1828

16. Clypeal anteroventral area large and vertically deflected; elytron rugopunctate, striae indistinct ...... Anomonyx Saylor, 1940

- Clypeal anteroventral area large and horizontal (Fig. 12K); elytral striae punctate Philochloenia Dejean, 1833

17. Mesoscutum-scutellum limit angulate (similar to Fig. 6G, detail b) .18

- Mesoscutum-scutellum limit not evident (Fig. 6H)

18. Protibia lateroproximal margin serrate Pristerophora Harold, 1869

- Protibia lateroproximal margin straight

19. Pronotal posterior margin medially prominent and with a small tooth (Fig. 2A-D) .......20

- Pronotal posterior margin unarmed, prominent or not

20. Protibial spur present Hieritis Burmeister, 1855

- Protibial spur absent 
21. Tarsus short, protarsomeres II-IV wider than long; male protarsomere I with an internodistal acute angle (Fig. 2B) ....................................................... Byrasba Harold, 1869

- Tarsus long, male protarsomeres I-II longer than wide; protarsomere I sometimes ventrally flattened, but without acute angle (Fig. 2A, 2C) .......................................... 22

22. Male metatibia internodistally flattened (Fig. 2E); female elytron with posterior margin widely beaded (Fig. 1B)

Agaocnemis Moser, 1918

- Male metatibia with an internodistal tooth (Fig. 2G); female elytron with posterior margin finely beaded Hamatoplectris Frey, 1967

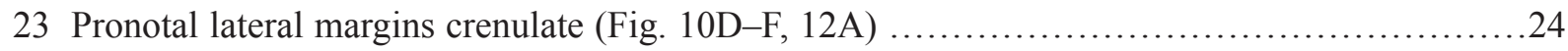

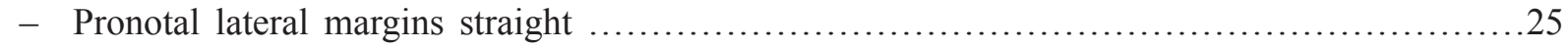

24. Clypeus semicircular, and narrow; pronotal posterior margin widely prominent (Fig. 12A) ...... Euryaspis Blanchard, 1850

- Clypeus trapezoid or rectangular, that of male of some species with large and acute projections; pronotal posterior margin weakly prominent (Fig. 10D-F)

Rhinaspis Perty, 1833

25. Pronotal posterior margin with two small posterior projections extended over the elytronscutellum contact (Fig. 13A) ...........................Pseudoserica Guérin-Méneville, 1838

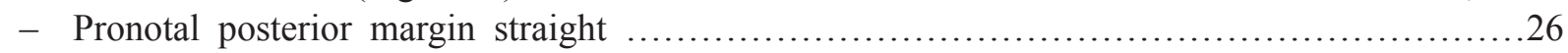

26. Male clypeus with two long horns (Fig. 10B-C); female clypeus deeply emarginate (Fig. 10A); clypeal posterior angle acute and partially covering the canthus ...Ceratolontha Arrow, 1948

- Clypeus semicircular, trapezoid or subrectangular, anterior angle variable, but never forming a long horn; clypeal posterior angle extended or not over the canthus, but never acute ...27

27. Clypeus large, anteroventral area large and horizontal (Fig. 3A), posterior angle partially covering the canthus (Fig. 3B); metafemur with dense short setae and some sparse long setae (Fig. 3C) ... Alvarinus Blanchard, 1850

- Clypeus short and with reduced anteroventral area (similar to Fig. 11H) OR clypeus large with posterior angle not extended over the canthus and femur with homogeneous thin setae ...28

28. Protibia with two external teeth and without spur; metacoxa and metafemur wide, metafemur internal side straight and external side strongly prominent .............................29

- Protibia with 1-4 external teeth and with or without spur; metacoxa and metafemur narrow, metafemur external side straight or weakly prominent .30

29. Antennae with 8-10 antennomeres, when antennae with eight antennomeres the pronotum and pygidium have small and sparse punctures (punctures separated by more than twice the puncture diameter) ...........................Dicrania LePeletier de Saint-Fargeau \& Audinet-Serville, 1828

- Antenna with eight antennomeres, pronotum and pygidium with large and dense punctures (punctures separated by less than a puncture diameter)

Canestera Saylor, 1938

30. Maxillary palpomere IV distinctly enlarged (Fig. 6A-B); antennomere VI longer than III (Fig. 6A) ... Clavipalpus Laporte, 1832

- Maxilla with palpomere IV not enlarged; antennomere III as long as IV (Fig. 6C) or longer than IV .31

31. Maxilla with palpomere IV twice as long as the width of palpomere III (Fig. 6C-D)

Paulosawaya Martínez \& d'Andretta, 1956

- Maxilla with palpomere IV 1.5 times longer than the width of palpomere III 


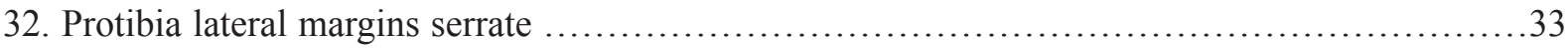

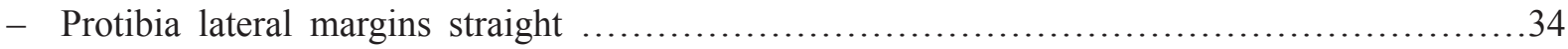

33. Total length greater than $9 \mathrm{~mm}$, protibial spur present .............Ptyophis Redtenbacher, 1868

- Total length less than $8 \mathrm{~mm}$, protibial spur absent ......Extenuoptyophis Smith \& Mondaca, 2015

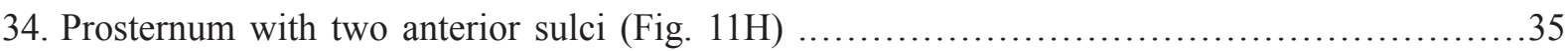

- Prosternum anteriorly concave (similar to Fig. 12K), or with longitudinal carina, or without

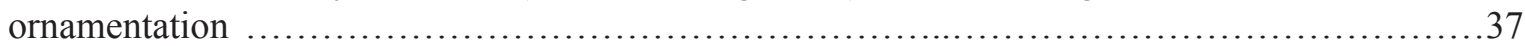

35 Pronotum as long as wide or longer than wide (Fig. 11C) .............Macrodactylus Dejean, 1821

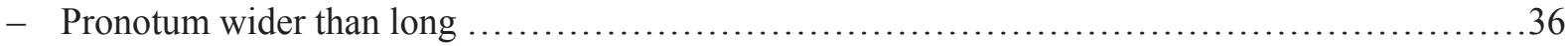

36. Supraocular area strongly angulate; pronotal disc glabrous (Fig. 11B) ....Manodactylus Moser, 1919

- Supraocular area slightly angulate; pronotal disc pubescent (Fig. 11A) ...Chariodactylus Moser, 1919

37. Meso-metaventrite medial contact prominent as a knob between mesocoxae .................38

- Meso-metaventrite medial contact knob absent or inconspicuous (inconspicuous in Issacaris, Phytholaema, but noted as a 90-degree surface deflection) ....................................41

38. Meso-metaventrite knob projecting forward to procoxae ...Modialis Fairmaire \& Germain, 1860

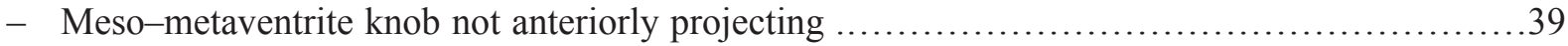

39. Protibial spur present

Pseudodicrania Gutiérrez, 1950

- Protibial spur absent

40 Eye large; clypeus broadly parabolic

Insimuloissacaris Smith \& Mondaca, 2015

- Eye small; clypeus quadrate .Neuquenodactylus Smith \& Mondaca, 2015

41. Meso-metaventrite medial contact separating the mesocoxae ...........Issacaris Fairmaire, 1889

- Mesocoxae subcontiguous

42. Clypeal anteroventral area broad; mentum as wide as long; prosternal anterior area narrow, and procoxal cavities and head-pronotum foramen subcontiguous ...Phytholaema Blanchard, 1851

- Clypeal anteroventral area reduced, and labrum subcontiguous to anterior clypeal margin; mentum evidently longer than wide; prosternal anterior area large, and procoxal cavities distinctly separate from head-pronotum foramen

43. Head with spine-like setae

Compsodactylus Fuhrmann, 2012

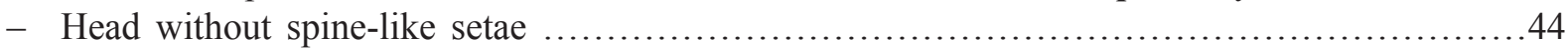

44. Protibial spur present; metatibia with two spurs; sometimes dorsal surface covered with scale-like

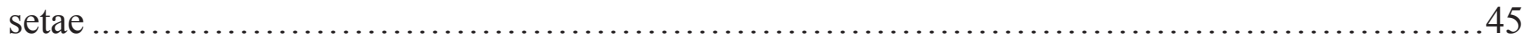

- Protibial spur absent; male metatibia without spur, female metatibia with 1-2 spurs; dorsal surface

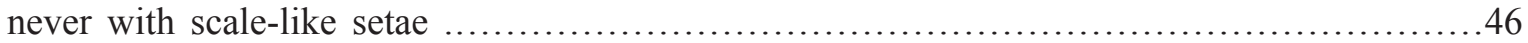

45. Body elongate; male ventrites II-V medially concave (Fig. 16A-C); female abdomen sinuous in lateral view (Fig. 16D)

Schizochelus Blanchard, 1850

- Body somewhat wide and oval; male abdomen without concave area; female abdomen simply curved in lateral view

Isonychus Mannerheim, 1829

46. Dorsal surface without obvious setal patterns, setae not prominent and evenly distributed

Pusiodactylus Smith, 2008

- Dorsal surface with obvious setal patterns, setae prominent or not evenly distributed

Ampliodactylus Smith, 2008 


\section{Discussion}

\section{Male morphology and nomenclature}

Some Macrodactylini genera are strongly sexually dimorphic, with males displaying remarkable interspecific variation and females with homogeneous morphology (e.g., Dicrania flavoscutellata Laporte, 1832, see Frey 1972: fig. 2; Compsodactylus martinezi (Frey, 1972), see Fuhrmann 2012). Sometimes conspicuous male characters are used to erect new genera. This procedure can split species, resulting in an unnecessary description of redundant taxa (monotypic genera) (if not artificial taxa, when cladistic analyses are used). A careful study of the females of these species having conspicuous males provides morphological evidence against such taxa splits. Based on this evidence, the present study proposes the synonymy of Aulanota with Philochloenia and of Gastrohoplus with Schizochelus; these cases are commented on below.

Philochloenia armata nom. nov. (= Aulanota sulcipennis) has the metatibial apex with a long spine and without spurs (Fig. 12J), otherwise other Philochloenia studied (P. castaneipennis comb. nov., P. rufipennis, P. sulcatulus, P. sulcipennis comb. nov. and two Philochloenia spp.) have the metatibial apex without spines and with two spurs. Moser (1924) used the particular characters of $P$. armata nom. nov. to describe the monotypic Aulanota, and noted the similarity of this genus with Philochloenia (as Anoplosiagum). The present study uses other characters to define Philochloenia (see genus diagnosis and remarks).

Following the present review, Schizochelus now includes three species: S. bicoloripes Blanchard, 1850, S. flavescens and S. mirabilis comb. nov. Males of these species have peculiar ornamentation of the tibia, tarsus and abdomen (Figs 15, 16G-L), but females present a homogeneous morphology. Despite the male leg and abdominal ornamentation, other male and female characters are used here for generic characterization (see Schizochelus diagnosis).

Another example is that of Rhinaspis aeneofusca, where the males have the metatibia internodistally flattened (Fig. 10G), a character not found in other Rhinaspis. Based on this conspicuous characteristic, Moser (1919b) erected Rhinaspoides to include this species, and noted the similarity between Rhinaspoides and Rhinaspis. Despite the particular male metatibia, Katovich (2008) proposed synonymy of Rhinaspoides with Rhinaspis, a synonym confirmed by the present study.

\section{Acknowledgements}

Thanks to Dr. Sônia A. Casari (MZSP), Joana Cristovão (BMNH), Andrew Smith (Canadian Museum of Nature, Canada), and two anonymous reviewers for their helpful suggestions; to Ayr Bello for trying the key; to Gabriel Biffi (MZSP) for helping with the photography; Holger Dombrow for helping with the Hercitis / Barybas taxonomic problem; Mariana A. Cherman (Universidade Federal do Paraná, Brazil) for her comments on Philochloenia; Gavin Broad for careful editing of this manuscript; and the curators cited in the material and methods. JF thanks Coordenação de Aperfeiçoamento de Pessoal de Nível Superior (CAPES) for grant support at the beginning of this project; Fundação de Amparo à Pesquisa do Estado de São Paulo (FAPESP) (proc. 2011/20001-6) for a doctorate grant. FZVM is a CNPq fellow and part of this work was funded by CNPq (Conselho Nacional de Desenvolvimento Científico e Tecnológico - 304925/2010-1, 202327/2013-2, 302997/2013-0, 405697/2013-9, 484035/2013-4, 400681/2014-5, 306745/2016-0). This research also received support from the Synthesys Project (http://synthesys3.myspecies.info/) which is financed by the European Community Research Infrastructure Action under the FP7 (GB-TAF 3855). 


\section{References}

Aguilar-Fernández P.G. 1980. VII. El cultivo de la caña de azúcar: 105-106. In: Aguilar-Fernández P.G. (eds) Apuntes sobre el controls biologico y el control integrado de las plagas agricolas en el Peru. Revista Peruana de Entomologia 23: 83-110.

Arrow G.J. 1913. Synopsis of the melolonthid genus Ancistrosoma, with descriptions of new species and an allied new genus. Annals and Magazine of Natural History (8) 12: 425-432. https://doi.org/10.1080/00222931308693420

Arrow G.J. 1920. On the Oriental members of the coleopterous group Macrodactylides (Melolonthidae). The Annals and Magazine of Natural History (9) 6: 441-455. Available from http://biodiversitylibrary.org/page/19240239 [accessed 29 Aug. 2017].

Arrow G.J. 1948. A horned melolonthine beetle from South America. Annals and Magazine of Natural History (12) 1: 371-375. https://doi.org/10.1080/00222934808653917

Ayquipa G.E. \& Cueva M.A. 1979. Nombres cientificos y comunes de los insectos que atacan a la caña de azucar en el Peru. Revista Peruana de Entomologia 22: 95-97.

Bates H.W. 1887. Biologia Centrali-Americana, Insecta, Coleoptera, Copridae, Aphodiidae, Orphnidae, Hybosoridae, Geotrupidae, Trogidae, Aclopidae, Chasmatopteridae, Melolonthidae. [1886-1890] 2 (2): 25-160.

Bentley J. \& Vilca P. 2001. La papa en Huánuco: Semilla y conocimiento popular sobre las plagas y enfermedades. Centro Internacional de la Papa, Lima.

Beutel R.G. \& Lawrence J.F. 2005. Coleoptera, Morphology: 23-28. In: Beutel R.G. \& Leschen R.A.B. (eds) Part 38. Coleoptera, beetles. Volume 1: Morphology and Systematics (Archostemata, Adephaga, myxophaga, Polyphaga partim). In: Kristensen N.P. \& Beutel R.G. (eds) Volume IV Arthropoda: Insecta. In: Kükenthal W. (founder), Beier M., Fischer M., Helmcke J.-G., Starck D. \& Wermuth H. (orgs) Handbook of Zoology. A natural History of phyla of the Animal Kingdom. Walter de Gruyter, Berlin New York.

Blackwelder R.E. 1944. Checklist of the coleopterous insects of Mexico, Central America, The West Indies, and South America. Part 2. Bulletin of the United States National Museum 185: 189-341.

Blanchard C.É. 1842. "Livraison 61”: fig. 11. In: Blanchard C.É. 1835-1847. [see below]

Blanchard C.É. 1845. "Livraison 78”: 113-152. In: Blanchard C.É. 1835-1847. [see below]

Blanchard C.É. 1835-1847. Insectes de l'Amérique méridionale, recueillis par Alcide d'Orbigny. In: d'Orbigny A. Voyage dans L'Amérique méridionale (le Brésil, la République orientale de l'Uruguay, la République argentine, la Patagonie, la République du Chili, la République de Bolivia, la République du Pérou), exécuté pendant les années 1826, 1827, 1828, 1829, 1830, 1831, 1832 et 1833. Tome VI, Partie 2: 57-222. Chez V. Levrault, Strasbourg. [1842: livraison 61: fig. 11; 1845: livraison 78: 113-152]. https://doi.org/10.5962/bhl.title.85973

Blanchard C.É. 1850. Classe des insectes. Ordre des coléoptères. In: Milne-Edwards H., Blanchard C.É. \& Lucas H. (eds) Catalogue de la collection entomologique du Muséum d'Histoire naturelle de Paris. Gide et Baudry, Paris.

Bousquet Y. 2016. Litteratura Coleopterologica (1758-1900): a guide to selected books related to the taxonomy of Coleoptera with publication dates and notes. ZooKeys 587: 1-776. https://doi.org/10.3897/zookeys.583.7084

Bousquet Y. \& Bouchard P. 2013. The genera in the second catalogue (1833-1836) of Dejean's Coleoptera collection. ZooKeys 282: 1-219. https://doi.org/10.3897/zookeys.282.4401 
Brill N.L. \& Abney M.R. 2013. Plectris aliena (Coleoptera: Scarabaeidae): A new invasive soil pest in North Carolina agro-ecosystems. Journal of Integrated Pest Management 4: 1-8. https://doi.org/10.1603/IPM13006

Burmeister H. 1855. Handbuch der Entomologie. Vierter Band. Besondere Entomologie. Fortsetzung. Zweite Abtheilung. Coleoptera Lamellicornia Phyllophaga chaenochela. Theodor Johann Christian Friedrich Enslin, Berlin. https://doi.org/10.5962/bhl.title.8135

Cambefort Y. 2006. Des coléoptères, des collections \& des hommes. Muséum national d'Histoire naturelle, Paris.

Cherman M.A., Morón M.A. \& Almeida L.M. 2016. Phylogenetic relationships within Diplotaxini Kirby (Coleoptera: Melolonthidae: Melolonthinae) with emphasis on Liogenys Guérin-Méneville. Systematic Entomology 41: 744-770. https://doi.org/10.1111/syen.12188

Chevrolat A. 1847. Philochlaenia. In: d'Orbigny C.D. Dictionnaire universel d'histoire naturelle, résumant et complétant. Tous les faits présentés par les Encyclopédies, les anciens dictionnaires scientifiques, les Oeuvres complètes de Buffon, et les meilleurs traités spéciaux sur les diverses branches des sciences naturelles; - Donnant la description des étres et des divers phénomènes de la nature, l'étymologie et la définition des noms scientifiques, et les principales applications des corps organiques et inorganiques à l'agriculture, à la médecine, aux arts industriels, etc. Tome neuvième: 735-736 [9]. M.M. Renard \& Martinet et C., Paris. https://doi.org/10.5962/bhl.title.23115

Cisneros F.V. 1995. Control de Plagas Agrícolas. 2da Edition. Centro Internacional de la Papa, Lima.

Conte de Castelnau M. 1840. Histoire naturelle des insectes coléoptères, avec une introduction renfermant l'anatomie et la physiologie dês animaux articulés. In: Brullé A. (ed.) Histoire naturelle des animaux articulés, Annelides, Crustacés, Arachnides, Myriapodes et Insectes. tome deuxième. P. Duménil, Paris. [sometimes attributed to Laporte F.L. de]. https://doi.org/10.5962/bhl.title.35290

Dalla Torre K.W. 1913. Melolonthinae IV, pars 50. In: Schenkling S. \& Junk W. (eds) Coleopterorum catalogus. Vol. 20 Scarabaeidae. W. Junk, Berlin.

Dejean P.F.M.A. 1833-1836a. Catalogue des Coléoptères de la collection de M. le Comte Dejean. Méquignon-Marvis, Paris. [1833: livraison 1-2: 1-176; 1834: livraison 3: 177-256; 1835: livraison 4: 257-360; 1836a: livraison 5: 361-443].

Dejean P.F.M.A. 1836b-1837. Catalogue des coléoptères de la collection de M. le comte Dejean. Troisième édition, revue, corrigée et augmentée. Méquignon-Marvis. [1836b: livraison 1-4: 1-384; 1837: livraison 5: 385-503].

Desmarest E. 1860. Coléoptères. Buprestiens, scarabaéiens, piméliens, curculioniens, scolytiens, chrysoméliens, etc. Troisième partie. In: Chenu J.C. (ed.) Encyclopédie d'histoire naturelle ou traité complet de cette science d'après les travaux des naturalistes les plus éminents de tous les pays et de toutes les époques Buffon, Daubenton, Lacépède, G. Cuvier, F. Cuvier, Geoffroy Saint-Hilaire, Latreille, de Jussieu, Brongniart, etc., etc. Ouvrage résumant les observations des auteurs anciens et comprenant toutes les découvertes modernes jusqu'à nos jours. Marescq \& Compagnie, Paris.

Dubois A. 2008. Authors of zoological publications and nomina are signatures, not persons. Zootaxa 1771: 63-68.

Egúsquiza-Bayona R., Mendonza-Vereau Y. \& Salirras-C.E. 2006. Amenazas y planes de mitigación de cultivos nativos y sus parientes silvestres. Conservación in situ de los Cultivos Nativos y sus Parientes Silvestres 2006: 1-183. 
Erichson W.F. 1848. Bericht über die wissenschaftlichen Leistungen in der Naturgeschichte der Insecten, Arachniden, Crustaceen u Entomostraceen während des Jahres 1847. Archiv für Naturgeschichte 14: $27-140$.

Evans A.V. 2003. A checklist of the New World chafers (Coleoptera: Scarabaeidae: Melolonthinae). Zootaxa 211: 1-458.

Evans A.V. \& Smith A.B.T. 2005. An Electronic Checklist of the New World Chafers (Coleoptera: Scarabaeidae: Melolonthinae), Version 1. University of Nebraska State Museum, Lincoln. Available from http://digitalcommons.unl.edu/entomologypapers/2/ [accessed 13 Apr. 2008].

Evans A.V. \& Smith A.B.T. 2007. An Electronic Checklist of the New World Chafers (Coleoptera: Scarabaeidae: Melolonthinae), Version 2. University of Nebraska State Museum, Lincoln. Available from http://www.museum.unl.edu/research/entomology/nwmelos.htm [accessed 13 Apr. 2008].

Evans A.V. \& Smith A.B.T. 2009. An Electronic Checklist of the New World Chafers (Coleoptera: Scarabaeidae: Melolonthinae), Version 3. University of Nebraska State Museum, Lincoln. Available from http://www.museum.unl.edu/research/entomology/nwmelos.htm [accessed 22 Feb. 2010].

Fabricius J.C. 1801. Systema eleutheratorum. Secundum ordines, genera, species; adiectis synonymis, locis, observationibus, descriptionbus. Tome I. Bibliopoli Academici Novi, Kiel.

Frey G. 1964. Neue Melolonthiden (Col.). Entomologische Arbeiten aus dem Museum G. Frey 15: 691-701.

Frey G. 1967. Die Gattung Plectris (Philochlaenia). (Coleoptera, Melolonthinae). Entomologische Arbeiten aus dem Museum G. Frey 18: 1-136.

Frey G. 1972. Bestimmungstabelle der Gattung Dicrania Serv. (Coleoptera - Melolonthinae Macrodactylini). Entomologische Arbeiten aus dem Museum G. Frey 23: 67-85.

Frey G. 1975. Bestimmungstabelle der südamerikanischen Arten der Gattung Phyllophaga Harris und ihrer Untergattung Phytalus Er. (Col. Melolonthidae). Entomologische Arbeiten aus dem Museum G. Frey 26: 201-226.

Fuhrmann J. 2012. Compsodactylus, a new South American genus with one new species and two new combinations (Coleoptera: Scarabaeidae: Melolonthinae). Zootaxa 3577: 43-57.

Gemminger M. \& Harold B. 1869. Catalogus Coleopterorum hucusque descriptorum synonymicus et systematicus. Tome 4. Scarabaeidae. Sumptu E. H. Gummi, Munich.

Germar E.F. 1824. Insectorum species novae aut minus cognitae, descriptionibus illustratae. Vol. 1, Coleoptera. Hendel \& Sons, Halle.

Gess S.K. \& Gess F.W. 2014. Wasps and bees in southern Africa. Biodiversity Series 24, The South African National Biodiversity Institute, Durban.

Guérin-Méneville F.É. 1830-1831. Atlas. Insects. In: Duperrey M.L. Voyage autour du Monde exécuté par ordre du roi, sur la corvette de Sa Majesté, la Coquille, pendant les années 1822, 1823, 1824, et 1825, sous le ministère et conformément aux instructions de S.E.M. Le Marquis de Clermont-Tonnerre, ministre de la marine; et publié sous les auspices de son Excellence Mgr le Cte De Chabrol, Ministre de la Marine et des Colonies. Livraison 17-27. Arthur Bernard, Paris. [14 bis] [1831: livraison 20: fig. 3] https://doi.org/10.5962/bhl.title.57936

Guérin-Méneville F.É. 1838. Chapitre XIII. Insectes. In: Duperrey M.L. Voyage autour du Monde exécuté par ordre du roi, sur la corvette de Sa Majesté, la Coquille, pendant les années 1822, 1823, 1824, et 1825, sous le ministère et conformément aux instructions de S. E. M. Le Marquis de Clermont-Tonnerre, ministre de la marine; et publié sous les auspices de son Excellence Mgr le Cte 
De Chabrol, Ministre de la Marine et des Colonies. Tome 2. Partie 2: 57-319. Arthur Bernard, Paris. https://doi.org/10.5962/bhl.title.57936

Guimarães J.H. 1977. Host-parasite and parasite-host catalogue of South American Tachinidae (Diptera). Arquivos de Zoologia 28: 1-131.

Gutiérrez, R. 1952. Notas sobre Scarabaeidae neotrópicos (III). Revista Chilena de Entomologia 2: 207-227.

Harold E. von 1869. Abänderungen vergebener Namen. Coleopterische 5: 122-125.

Horn W., Kahle I., Friese G. \& Gaedike R. 1990a. Collectiones entomologicae. Ein Kompendium über den Verbleib entomologischer Sammlungen der Welt bis 1960. Teil I: A bis K. Akademie der Landwirtschaftswissenschaften der Deutschen Demokratischen Republik, Berlin.

Horn W., Kahle I., Friese G. \& Gaedike R. 1990b. Collectiones entomologicae. Ein Kompendium über den Verbleib entomologischer Sammlungen der Welt bis 1960. Teil II: L bis Z. Akademie der Landwirtschaftswissenschaften der Deutschen Demokratischen Republik, Berlin,.

Katovich K. 2008. A generic-level phylogenetic review of the Macrodactylini (Coleoptera: Scarabaeidae: Melolonthinae). Insecta Mundi 23: 1-78.

Katovich K. 2011. Pseudopectinosoma Katovich, new genus, and review of Pectinosoma Arrow (Scarabaeidae: Melolonthinae: Macrodactylini). The Coleopterists Bulletin 65: 335-340. https://doi.org/10.1649/072.065.0402

ICZN. 1999. International Code of Zoological Nomenclature, Fourth Edition, adopted by the International Union of Biological Sciences. International Trust for Zoological Nomenclature, London.

Lacordaire J.T. 1856. Histoire naturelle des insectes. Genera des Coléoptères, or exposé méthodique et criticque de tous genres proposés jusquici dans cet odre d'insectes. Tome Troiséme. Contenant les familles des Pecticornes et Lamellicornes. Librarie Encyclopédique de Roret, Paris. https://doi.org/10.5962/bhl.title.8864

Lacroix M. 2007. Pachydeminae du Monde (Scarabaeoidea, Melolonthidae) genera et catalogue commenté. Collection Hannetons 1. Éditions M. Lacroix, Romans sur Isère, France.

Laporte F.L. de. 1832. Mémoire sur cinquante espèces nouvelles ou peu connues d'insectes. Annales de la Société Entomologique de France 1: 386-415.

Lawrence J.F., Beutel R.G., Leschen R.A.B. \& Slipinski A. 2010. Glossary of morphological terms: 9-20. In: Leschen R.A.B., Beutel R.G. \& Lawrence J.F. (eds) Part 39. Coleoptera, Beetles. Volume 2: Morphology and Systematics (Elateroidea, Bostrichiformia, Cucujiformia partim). In: Kristensen N.P. \& Beutel R.G. (eds) Volume IV Arthropoda: Insecta. In: Kükenthal W. (founder), Beier M., Fischer M., Helmcke J.-G, Starck D. \& Wermuth H. (orgs) Handbook of Zoology. A natural History of phyla of the Animal Kingdom. Walter de Gruyter, Berlin-New York.

LePeletier de Saint-Fargeau A.L.M. \& Audinet-Serville J.G. 1828. Scarabé, Scarabaeus. In: Latreille, LePeletier de Saint Fargeau, Serville \& Guérin-Méneville. (eds). 1825-1828. Tome 10. Part 2. In: Latreille (ed.). Histoire naturelle. Entomologie, ou histoire naturelle de crustacés, des arachnides et des insectes. In: Encyclopédie méthodique, ou par ordre de matières; par une société de gens de lettres, de savants et d'artistes; précédée d'un vocabulaire universel, servant de table pour tout l'ouvrage, ornée des portraits de MM. Diderot et d'Alembert, premiers éditeurs de l'Encyclopédie: 346-382. Chez Mme Veuve Agasse, Paris, 832 p + [1 errata]. [1825: tome 10, part 1, 1-344; 1828: tome 10, part 2, 345-832].

López-García M.M., García-Atencia S. \& Amat-García G. 2015. Escarabajos fitófagos (Coleoptera: Scarabaeidae "Pleurosticti") de los Andes Orientales de Colombia (Departamentos de Santander, Boyacá 
y Cundinamarca). Boletín Centífico, Centro de Museos, Museo de Historia Natural [Boletín Científico del Centro de Museos de la Universidad de Caldas] 19: 320-358.

Martínez A. 1972. Algunas consideraciones sobre los generos Apterodema Fairmaire y Sericoides Guérin, con descripcion de dos nuevas especies (Scarab. Sericinae, Liparetrini). Comunicaciones del Museo Argentino de Ciencias Naturales Bernardino Rivadavia 1: 37-62.

Martínez A. \& d'Andretta M.A.V. 1956. Dos gêneros e espécies novos de Pachydemini do Equador (Col. Scarabaeoidea, Melolonthidae). Papéis Avulsos do Departamento de Zoologia 12: 345-356.

Medvedev S.I. 1952. Fauna USSR: Coleoptera 10, Nr. 1: Lamellicornia (Scarabaeidae), subfam. Melolonthinae part 2. Izd-vo Akademii Nauk SSSR 52, Akademii Nauk SSSR, Prague.

Mera-Velasco Y.A., Gallego-Ropero M.C. \& Armbrecht I. 2010. Interacciones entre hormigas e insectos en follaje de cafetales de sol y sombra, Cauca-Colombia. Revista Colombiana de Entomologia 36: $116-126$.

Mondaca J. \& OcampoF. 2012. Revision of the Chilean genus Ptyophis (Scarabaeidae: Mololonthinae[sic]: Macrodactylini). Revista Chilena de Entomologia 37: 47-60.

Morales-Valles P., Cermeli M., Godoy F. \& Salas B. 2003. Lista de insectos relacionados a las solanáceas ubicados en el Museo de Insectos de Interés Agrícola del CENIAP - INIA. Entomotropica 18: 193-209.

Moser J. 1913. Beitrag zur Kenntnis der Melolonthiden I. Deutsche Entomologische Zeitschrift 3: 271-297.

Moser J. 1918. Neue amerikanische Melolonthiden (Col.). Stettiner Entomologische Zeitung 79: 95-167.

Moser J. 1919a. Beitrag zur Kenntnis der Melolonthiden (Col.). (IX). Stettiner Entomologische Zeitung 80: 3-64.

Moser J. 1919b. Beitrag zur Kenntnis der Melolonthiden. (Col.) (X.). Stettiner Entomologische Zeitung 80: 330-364.

Moser J. 1921. Neue Melolonthiden Mittel- und Süd-Amerikas. Stettiner Entomologische Zeitung 82: $133-182$.

Moser J. 1924. Beitrag zur Kenntnis der Melolonthiden (Col.). (XIV). Stettiner Entomologische Zeitung 84: 137-164.

Neita-Moreno J.C., Easdale C., Salvatore A.R. \& Ocampo F.C. 2012. Descripción de los estados inmaduros de Ancistrosoma argentinum Moser (Scarabaeidae: Melolonhtinae: Macrodactylini), con notas sobre la biología, distribución e importancia agrícola en Argentina. IX Reunión Latinoamericana de Scarabaeoidologia, programas y resúmenes: 42.

Nonfried A.F. 1894. Beiträge zur Coleopterenfauna von Ostasien und Polynesien. Entomologische Nachrichten 20: 9-14.

Ohaus F. 1909. Bericht über eine entomologische Studienreise in Südamerika. Stettiner Entomologische Zeitung 70: 3-139.

Ohaus F. 1918. Scarabaeidae: Euchirinae, Phaenomerinae, Rutelinae, pars 66: 1-241. In: Schenkling S. \& Junk W. (eds) Coleopterorum catalogus 20, W. Junk, Berlin.

Paucar-Cabrera A. 2003. Systematics and phylogeny of the genus Epectinaspis Blanchard (Coleoptera: Scarabaeidae: Rutelinae) and description of a new genus of Anomalini from Mexico. The Coleopterists Bulletin 57: 3-60. https://doi.org/10.1649/0010-065X(2003)57[3:SAPOTG]2.0.CO;2

Peck S.R., Cook J. \& Hardy Jr. J.D. 2002. Beetle fauna of the insland of Tobago, Trinidad and Tobago, West Indies. Insecta Mundi 16: 9-23. 
Peña J.E. \& Bennet F.D. 1995. Arthropods associated with Annona spp. in the Neotropics. Florida Entomologist 78: 329-349.

Perty J.A.M. 1833. Delectus animalium articulatorum, quae in itinere per Brasiliam annis MDCCCXVIIMDCCCXX jussu et auspiciis Maximiliani Josephi I. Bavariae regis augustissimi peracto. Fascicle 1. Hübschmann, Munich.

Prokofiev A.M. 2015. Genus Dichelomorpha Burmeister, 1855 in the fauna of Vietnam: species with abbreviated 1-4 mesotarsal segments in males (Coleoptera, Scarabaeidae: Melolonthinae: Diphycerini). Eurasian Entomological Journal 14: 543-551.

Ratcliffe B.C., Jameson M.L., Figueroa L., Cave R.D., Paulsen M.J., Cano E.B., BezaBeza C., Jimenez-Ferbans L. \& Reyes-Castillo P. 2015. Beetles (Coleoptera) of Peru: a survey of the Families. Scarabaeoidea. Journal of the Kansas Entomological Society 88: 186-207. https://doi.org/10.2317/kent-88-02-186-207.1

Reed E.C. 1872. Observaciones sobre los Coleópteros chilenos descritos por el señor doctor Redtenbacher. Anales de la Universidad de Chile 1872 [41]: 190-196.

Reed E.C. 1876. Catálogo de los coleópteros de Chile. Segunda parte. Anales de la Universidad de Chile 1876 [48]: 274-295.

Restrepo-Giraldo H. \& López-Ávila A. 2000. Especies de Chisas (Coleoptera: Melolonthidae) de importancia agrícola en Colombia. Corporación Colombiana de investigación agropecuaria, Bogota.

Roberts R.J. 1968. An introduced pasture beetle, Plectris aliena Chapin (Scarabaeidae: Melolonthinae). Australian Journal of Entomology 7: 15-20.

Rogg H.W. 2000. Manual de entomología agrícola do Ecuador. Abya-Yala, Quito.

Rojo-Jiménez E. 2014. Café I (G. Coffea). Reduca (Biología), Serie Botánica 7: 113-132.

Ruiz-Mazanos E. 2006. Redescription and first record of Diaphylla granulata for Argentina (Coleoptera: Scarabaeidae: Melolonthinae). Revista de la Sociedad Entomológica Argentina 65: 79-86.

Rühl F. 1888-1892. Beitrag zur Charakteristik der Lamellicornien. Societas Entomologica 3 (6): 43-44, 3 (7): 52-53, 3 (8): 60, 3 (10): 73-74, 3 (12): 90, 3 (14): 107-108, 3 (15): 116-117, 3 (17): 129-130, 3 (19): 146-147, 3 (22): 171-172, 4 (7): 59-60, 4 (8): 67, 4 (9): 74-75, 4 (11): 89-90, 4 (12): 98, 5 (22): 172-173, 5 (24): 186-187, 6 (1): 3, 6 (3) [not seen], 6 (7): 53, 6 (8): 60-61, 6 (9): 67-68, 6 (11): 82, 6 (14): 108-109, 6 (16): 124, 6 (21): 164-165, 6 (22): 173. [1888: n.3 (fasc.1-18); 1889: 3 (19-24), 4 (1-18); 1890: 4 (19-24), 5 (1-18); 1891: 5 (19-24), 6 (1-18); 1892: 6 (19-24)] [Sciuropus 1888, 3 (6): 44]

Santandeu A. 2010. Capítulo 2. Biopreparados probados en la agricultura urbana y periurbana sostenible. In: IPES \& FAO. Biopreparados para el manejo sostenible de plagas y enfermedades en la agricultura urbana y periurbana. Guías ?Cómo Hacerlo? 3: 1-93.

Saylor L.W. 1940. Two new generic names for South American Coleoptera. Proceedings of the Entomological Society of Washington 42: 46.

Smith A.B.T. 2002. Revision of the southern South American endemic genus Aulacopalpus Guérin-Méneville with phylogenetic and biogeographic analyses of the Subtribe Brachysternina (Coleoptera: Scarabaeidae: Rutelinae: Anoplognathini). The Coleopterists Bulletin 56: 379-437. https://doi.org/10.1649/0010-065X(2002)056[0379:ROTSSA]2.0.CO;2

Smith A.B.T. 2008. South American Melolonthinae (Coleoptera: Scarabaeidae) classification and nomenclature: some problems and solutions. Insecta Mundi 60: 1-28. 
Smith A.B.T. 2016. Corrections to Neotropical Rhinaspis Perty and Plectris LePeletier and AudinetServille (Coleoptera: Scarabaeidae: Melolonthinae) classification and nomenclature. Insecta Mundi 0463: 1-6.

Smith A.B.T. \& Evans A.V. 2005. A supplement to the checklist of the New World chafers (Coleoptera: Scarabaeidae: Melolonthinae) with notes on their tribal classification. Zootaxa 1032: 29-60.

Smith A.B.T. \& Mondaca J. 2015. Review of the southern South American Macrodactylini (Coleoptera: Scarabaeidae: Melolonthinae) with descriptions of new genera and species. Zootaxa 4056: 1-65. https://doi.org/10.11646/zootaxa.4056.1.1

Smith A.B.T., Hawks D.C. \& Heraty J.M. 2006. An overview of the classification and evolution of the major scarab beetle clades (Coleoptera: Scarabaeoidea) based on preliminary molecular analyses. The Coleopterists Bulletin 60: 35-46. https://doi.org/10.1649/0010-065X(2006)60[35:AOOTCA]2.0.CO;2

Valoy M.E., Bruno M.A., Prado F.E. \& González J.A. 2011. Insects associated to a quinoa crop in Amaicha del Valle, Tucumán, Argentina. Acta Zoologica Lilloana 55: 16-22.

Valoy M., Reguilón C. \& Podazza G. 2015. Chapter 5. The potential of using natural enemies and chemical compounds in quinoa for biological control of insect pests. In: Murphy K. \& Matanguihan J. (eds) Quinoa: improvement and sustainable production: 63-86. John Wiley \& Sons Inc., Hoboken.

Valdivieso-Jara L. \& Núñez-Sacarías E. 1984. Plagas del maiz y sus enemigos naturales. Instituto Nacional de Investigación y Promoción Agropecuaria, Manual Técnico 4: 1-66.

Manuscript received: 3 May 2016

Manuscript accepted: 19 December 2016

Published on: 13 September 2017

Topic editor: Gavin Broad

Desk editor: Kristiaan Hoedemakers

Printed versions of all papers are also deposited in the libraries of the institutes that are members of the EJT consortium: Muséum national d'Histoire naturelle, Paris, France; Botanic Garden Meise, Belgium; Royal Museum for Central Africa, Tervuren, Belgium; Natural History Museum, London, United Kingdom; Royal Belgian Institute of Natural Sciences, Brussels, Belgium; Natural History Museum of Denmark, Copenhagen, Denmark; Naturalis Biodiversity Center, Leiden, the Netherlands; Museo Nacional de Ciencias Naturales-CSIC, Madrid, Spain; Real Jardín Botánico de Madrid CSIC, Spain. 